\title{
VALIDATION OF MODELS FOR SHOCK-DRIVEN HYDRODYNAMIC INSTABILITIES
}

\author{
A Thesis presented to \\ the Faculty of the Graduate School \\ at the University of Missouri
}

In Partial Fulfillment

of the Requirements for the Degree

Doctor of Philosophy

by

SAHIR R. ALMUHNA

Dr. Jacob A. McFarland, Dissertation Supervisor

July 2021 
The undersigned, appointed by the Dean of the Graduate School, have examined the dissertation entitled:

\section{VALIDATION OF MODELS FOR SHOCK-DRIVEN HYDRODYNAMIC INSTABILITIES}

presented by Sahir Rakim Almuhna, a candidate for the degree of Doctor of Philosophy and hereby certify that, in their opinion, it is worthy of acceptance.

Dr. Hongbin Ma

Dr. Gary L. Solbrekken

Dr. Angela Speck

Dr. Jacob A. McFarland

Dr. Matthew R. Maschmann 


\section{ACKNOWLEDGMENTS}

I don't know from where to start with thanking who are around me. Should I start with my great mentor and advisor Dr. McFarland, with my wife who spent six years from here life leaving her job and her family and taking care to the kids in multicultural environment, my Mom who prays every day for me such that God (Allah) save my family and me, or my lab mates Roy, John, Vasco, Curtis, Hanif \& Calvin, who don't hesitate to help when they are asked.

I might need to start with my country, Iraq, for its support for many years while I am living in the USA, but what about NSF for their support and assistance in my research, or the University of Missouri for a pleasant learning environment and access to different learning tools.

I worked with many advisors, but I ended up working with Dr. McFarland, who spent much time for guiding and helping me. I tried to follow a path that build background information in any area I am interested in, so that I relay on books more than papers, because the papers didn't give you the whole picture. Fortunately, the track of Dr. McFarland which draws to me fits with what I think would be correct to my plan. Working with Dr. Mcfarland doesn't mean that the other advisors were not helpful. However, they were helpful with making a right choice for my self, for example, Dr. Ma advise me to work with people who do CFD after a while working with him in heat pipe project, he was honest with his advice, and the other were also same.

In the end, I would like to thank Dr. Ma, who taught me phase change, Dr. Solbrekken, for CFD class, Dr. Angela Speck, for her helps in the Astrophysics part of the research and Dr. Maschmann for teaching Nanoscale Energy Transport class.

Sahir, as a human being, some time forget forgets the people who are close to him. My wife who scarifies with here job for many years to take care to the kids, and taking care to all of us. In fact, I declare my disability to thank her. 
TABLE OF CONTENTS

ACKNOWLEDGMENTS ................ ii

LIST OF TABLES $\ldots \ldots \ldots \ldots \ldots \ldots$ vi

LIST OF FIGURES $\ldots \ldots \ldots \ldots \ldots \ldots \ldots$ vii

ABSTRACT .................. ix

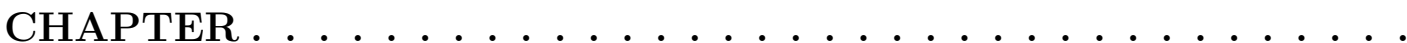

1 Introduction ......................... 1

1.1 Hydrodynamic Instability $\ldots \ldots \ldots \ldots \ldots$

1.1.1 Kelvin-Helmholtz Instability (KHI) . . . . . . . . . . . 3

1.1.2 Rayleigh-Taylor Instability (RTI) . . . . . . . . . . . 4

1.1.3 Richtmyer-Meshkov Instability (RMI) $\ldots \ldots \ldots \ldots$

1.1.4 Shock- Driven Multi-Phase Flow Instability (SDMI) . . . . . 8

1.1.5 Shock- Driven Multi-Phase Flow with Radiation Instability . . 8

1.2 Mechanism of SDMI . . . . . . . . . . . . . . . . . . 11

1.3 Mechanism of SDMI at circumstellar medium . . . . . . . . . 13

1.4 Challenges and Goals of Current Research . . . . . . . . . . . . 14

1.5 Accomplished Tasks in This Research . . . . . . . . . . . . . 16

2 Literature Review . . . . . . . . . . . . . . . 17

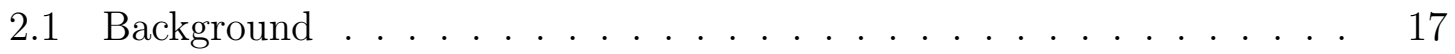

2.2 Multi-phase Particles in Cell Method (MP-PIC) . . . . . . . . . 19

2.3 Experimental Studies . . . . . . . . . . . . . . . . . . . . . . . . . 21

2.4 Hydrodynamic Simulations near AGB Stars . . . . . . . . . 22

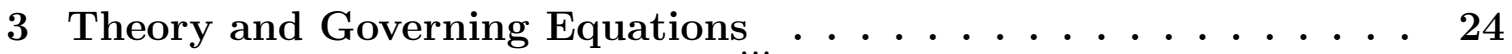


3.1 Background . . . . . . . . . . . . . . . . . . . . . . . . 24

3.1 .1 Mach Number $M_{a} \ldots \ldots \ldots \ldots \ldots \ldots \ldots$

3.1 .2 Effective Atwood Number $\left(A_{e}\right) \ldots \ldots \ldots \ldots$

3.1 .3 Perturbation of Interface $\ldots \ldots \ldots \ldots \ldots$

3.1.4 Reynolds number $(R e) \ldots \ldots \ldots \ldots$

3.1.5 Knudson Number $\left(K_{n}\right) \ldots \ldots \ldots \ldots \ldots$

3.2 Radiation Parameters . . . . . . . . . . . . . . . . . . . . . . . . . . . 29

3.2 .1 Radiation Intensity $\ldots \ldots \ldots \ldots$

3.2 .2 Radiation Pressure . . . . . . . . . . . . . . . . . . . 32

3.2 .3 Radiation Acceleration . . . . . . . . . . . . . . . . . . 34

3.3 Gravity . . . . . . . . . . . . . . . . . . . . 34

3.4 Governing Equations . . . . . . . . . . . . . . . . . . 35

3.4 .1 Eulerian Gas System . . . . . . . . . . . . . . . . 35

3.4 .2 Lagrangian System . . . . . . . . . . . . . . . . . . 37

3.5 Drag Models . . . . . . . . . . . . . . . . . . . . . . . 40

3.5 .1 Continuum Model . . . . . . . . . . . . . . . . . . . . . . . 40

3.5 .2 Non-Continuum Model . . . . . . . . . . . . . . . . . . . . 41

4 Numerical and Experimental Configurations . . . . . . . . . . 44

4.1 Introduction $\ldots \ldots \ldots \ldots \ldots \ldots \ldots \ldots \ldots \ldots \ldots$

4.2 Building The Geometry _ . . . . . . . . . . . . . . 44

4.2 .1 Shocktube problem . . . . . . . . . . . . . . . . 44

$4.2 .2 \quad$ AGB Star Problem . . . . . . . . . . . . . . . 45

4.3 Grid Generation . . . . . . . . . . . . . . . . . . . 46

4.4 Initial and Boundary Conditions. . . . . . . . . . . . 47

4.5 Particles Generation. . . . . . . . . . . . . . . . . . 51 
4.6 Experimental Calculation of effective Atwood Number. . . . . . . . . 53

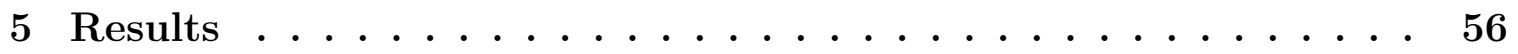

5.1 Introduction . . . . . . . . . . . . . . . 56

5.2 Simulation of the shocktube problem. . . . . . . . . . . 56

5.2.1 Comparisons with the experimental Results. . . . . . . . 58

5.3 Simulation of AGB Star Problem . . . . . . . . . . . . . 62

5.3.1 AGB stars Observations . . . . . . . . . . . . . 62

5.4 SDMI Simulation near AGB star . . . . . . . . . . . . 65

5.4.1 Radiation and gravity effects . . . . . . . . . . . . 68

6 Conclusions and Suggestion for Future Works . . . . . . . 70

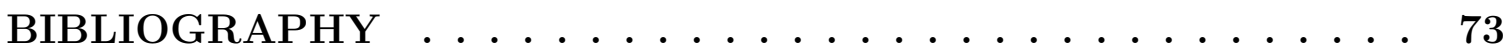

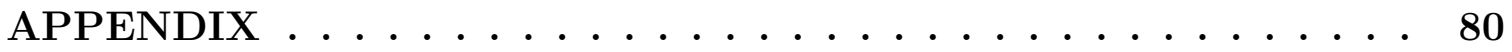

A Codes Implemented ..................... 80

A.1 One Dimensional Shock tube Analysis . . . . . . . . . . . . . . . 80

A.2 Calculations of Atwood Number . . . . . . . . . . . . . . 85

A.3 Non-Continuum Drag Model . . . . . . . . . . . . . . . . 93

A.4 Point mass Gravity Model . . . . . . . . . . . . . . . . 96

A.5 Input Parameters . . . . . . . . . . . . . . . . . 103

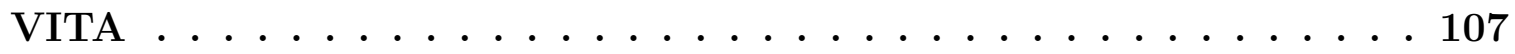




\section{LIST OF TABLES}

Table $\quad$ Page

5.1 Initial gas condition in shock-tube problem . . . . . . . . . . 57

5.2 Main Inputs in AGB star problem . . . . . . . . . . . . . . . . 66 


\section{LIST OF FIGURES}

Figure $\quad$ Page

1.1 Classification of $\mathrm{HI} \ldots \ldots \ldots \ldots \ldots \ldots$

1.2 Kelvin-Helmholtz instability . . . . . . . . . . . . . . 4

1.3 Rayleigh-Taylor Instability . . . . . . . . . . . . . . . 5

1.4 Rayleigh-Taylor Instability . . . . . . . . . . . . . . . . 6

1.5 RTandKH Instability $\ldots \ldots \ldots$

1.6 SDMI versus RTI . . . . . . . . . . . . . . . . . . . . . . . . . . 12

1.7 Schematics of AGB star envelops . . . . . . . . . . . . . 14

2.1 Classification of phase-coupling mechanisms . . . . . . . 18

2.2 classification of particles cloud approaches $\ldots \ldots \ldots \ldots$

3.1 SDMI's Required Inputs $\ldots \ldots \ldots \ldots$

3.2 Different Possible Interface . . . . . . . . . . . . . . . . . . 27

3.4 Radiation emitted by a surface $\ldots \ldots \ldots \ldots \ldots$

3.3 Radiation emitted by a surface $\ldots \ldots \ldots \ldots \ldots$

3.5 View Factor . . . . . . . . . . . . . . . . . . . . . 33

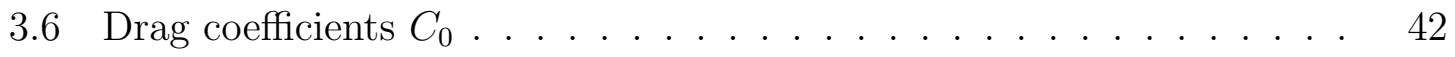

3.7 Drag coefficients ratio $C_{1} / C_{o} \ldots \ldots \ldots \ldots \ldots \ldots$

4.1 Actual Versus Computational size of Shock-tube . . . . . . . . . . 45

4.2 Simulation Domain of AGB star. . . . . . . . . . . . . 46 vii 
4.3 Simulation Domain of AGB star. . . . . . . . . . . . . 47

4.4 Levels of refinement near AGB star. . . . . . . . . . . . . . . 47

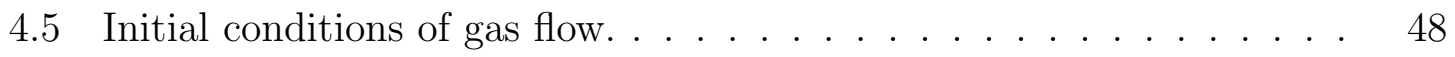

4.6 Initial conditions of gas flow. . . . . . . . . . . . . . . 49

4.7 Radiation Field. . . . . . . . . . . . . . . . . . . . . 50

4.8 Particles Distribution . . . . . . . . . . . . . . . . . . . . . . 52

4.9 AGB star particles Distribution . . . . . . . . . . . . . 53

4.10 Schematic of the shock tube facility in university of Missouri . . . . 54

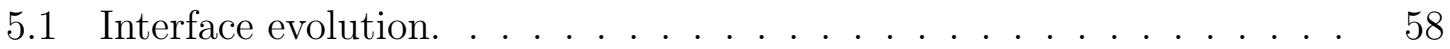

5.2 Interface evolution, first case. . . . . . . . . . . . . . . . . . 59

5.3 Interface evolution, second case. . . . . . . . . . . . 61

5.4 Near Agb star evolution, bow shocks. . . . . . . . . . . . 63

5.5 Near Agb star evolution, Concentric arcs. . . . . . . . . . . . 64

5.6 Near Agb star evolution, Rings. . . . . . . . . . . . . . . . 65

5.7 Simulation near AGB star evolution . . . . . . . . . . . . . 67 


\title{
VALIDATION OF MODELS FOR SHOCK-DRIVEN HYDRODYNAMIC INSTABILITIES
}

\author{
Sahir R. Almuhna \\ Dr. Jacob A. McFarland, Dissertation Supervisor
}

\begin{abstract}
The propagation of fluids through space-time is a truly beautiful and mysterious marvel that humankind has spent nearly all our existence trying to comprehend, understand, manipulate, and master. From waves over water to the Sun and the stars in the sky; fluids prove to be as elementary as they are esoteric, as calming as they are chaotic, and as delicate as they are detrimental. The levity in which fluids propagate can be as swift as the milliseconds it takes to observe hydrodynamic instability in say a shock tube facility, to the hundreds if not thousands of years over which a cosmological event's hydrodynamic instability may evolve. Comprehending, studying, manipulating, and mastering the propagation of fluids, specifically within the realm of fluid mechanics, s.c., hydrodynamic instability (HI), is of paramount prominence to the success of humankind. Today, a group of personnel within the scientific and academic community study the evolution and propagation of hydrodynamic instabilities (HIs) through a vast multitude of avenues for a plethora of applications; the two main avenues being experimentally and computationally. However, the ability to experimentally generate, for example, Asymptotic giant branch (AGB) star within a laboratory is as unattainable as the multiple lifetimes for its hydrodynamic instabilities take to develop and evolve, and study. The necessity of generating numerical simulations which match the experimental results of the growth and morphological evolution of hydrodynamic instabilities is a perfectly idealized way to address the capacious and enduring time scales of the hydrodynamic instabilities mentioned. The


goal of this dissertation work is to compare the numerical results of the evolution of HIs with experimental results, generate qualitative and quantitative analyses of how the results differ, and improve upon the numerical methods in which the simulation results are generated. To achieve the goal of this dissertation, the evolution and morphology of the two-dimensional hydrodynamic Shock-Driven Multiphase Instability (SDMI) is investigated through experimental measurements obtained within a shock tube facility. The experimental results are then used to validate the results achieved through simulations which utilize identical initialized parameters to model the experiment. The simulations were performed in the open source software FLASH, which is employed to solve the Multi-Phase Particle-in-Cell (MP-PIC) method with the Piecewise Parabolic Method (PPM) for the SDMI's multispecies gas flow. To gather data on the SDMI's morphological evolution experimentally, the planar laser Mie scattering (PLMS) technique was used to illuminate a cylindrical particle-laden flow field (interface), in 2-D, where high-resolution charged-coupled device (CCD) camera captured cross-sectional images of the interface's evolution. The gas flow itself consisted of a mixture of three different species: nitrogen, air, and water vapor; while the dispersed phase consists of water droplets in gas mixture. Utilizing a Mach number, $M_{a}$ of 1.67 , equivalent to a shock wave velocity, $v_{s h}$ of $570\left[\mathrm{~ms}^{-1}\right]$, data was obtained for two different effective Atwood numbers (particles concentrations), $A_{t}$ of 0.0479 and 0.0184 ,at three time intervals for comparison of the experimental data to the computationally acquired data. The results obtained from the computational and experimental data show good quantitative agreement. For example, average dispersed phase speed measured experimentally is $99.5 \%$ of average calculated speed numerically, also, shape wise numerical distance between two developed vortices in dispersed phase is $93.5 \%$ of those measured experimentally. Qualitatively, the morphology of the dispersed phase shows same evolution in both simulated and experimental results. SDMI can also be seen in the circumstellar medium with the infinite number of mor- 
phologies due to the complexity of the hydrodynamics evaluations near AGB stars. An attractive solution shows the pulsation of the AGB star producing hot bubble combined with a shock wave and then interacting with dust shell making different types of instabilities. 


\section{Chapter 1}

\section{Introduction}

Before we get into definitions of Hydrodynamic Instability (HI) terminology, we would love to introduce a question which is in mind of many engineers who work in computational fluid dynamics (CFD). Is the numerical solution code or software able to simulate the specific phenomenon? This question was the motivation of this research, but the question is general, and it needs many details to explore before getting the answer. So, this research explores numerical and experimental multi-physics phenomenon with a specific type of Hydrodynamic instability (HI) called shock-driven multiphase flow instability (SDMI). We will start with hydrodynamic instability definition in the first chapter. In the second chapter, we will go through a literature review of numerical and experimental studies. Chapter three will introduce governing equations and their discretization. In chapter four, the configuration of numerical and experimental methods is presented. Moreover, chapter five will present the results and suggestions for future work.

In the next section, we introduce the definition of HIs and their classification. 


\subsection{Hydrodynamic Instability}

Hydrodynamic Instability (HI) is a branch of fluid mechanics that deals with the onset of instability of fluid flow motion and how these instabilities evolve to turbulent flow. The definition above refers to the transitional region in fluid flow regimes which is transient between laminar and turbulent fluids. The previous definition is an older definition that fits with Osborne Reynolds' experiment. However it is more appropriate to quote James Clark Maxwell's declaration in 1882 that describes HI generally,

"When an infinitely small variation of the present state will alter only by an infinitely small quantity the state at some future time, the condition of the system, whether at rest or in motion, is said to be stable but when an infinitely small variation in the present state may bring about a finite difference in the state of the system in a finite time, the system is said to be unstable." [1]

Many scientists are attracted by HI from hundreds of years ago to present times. HI is seen in daily life, for example, when the wind blows the surface of the water, causing waves or hits clouds causing rollups on the interface regions. Some of HIs also happen in extreme condition changes like volcanic eruptions that cause mushroom clouds or cosmic dust processes that occur in outer space phenomena like the evolution of supernovae or planetary nebulae. Most cosmologist believes that HI plays an essential role in all cosmic evolution [2].

Different HIs rely on different mechanisms that cause those instabilities. To name a few, the Kelvin-Helmholtz Instability (KHI), which is driven by shear difference across fluid layers, and the Rayleigh-Taylor Instability (RTI), which is generated by density and pressure gradients with initial perturbation. Figure 1.1 lists most common HIs and the reasons behind their generations.

We can observe these HIs in natural or human made applications. RichtmyerMeshkov Instability (RMI) and Shock Driven Multiphase Flow Instability (SDMI) 


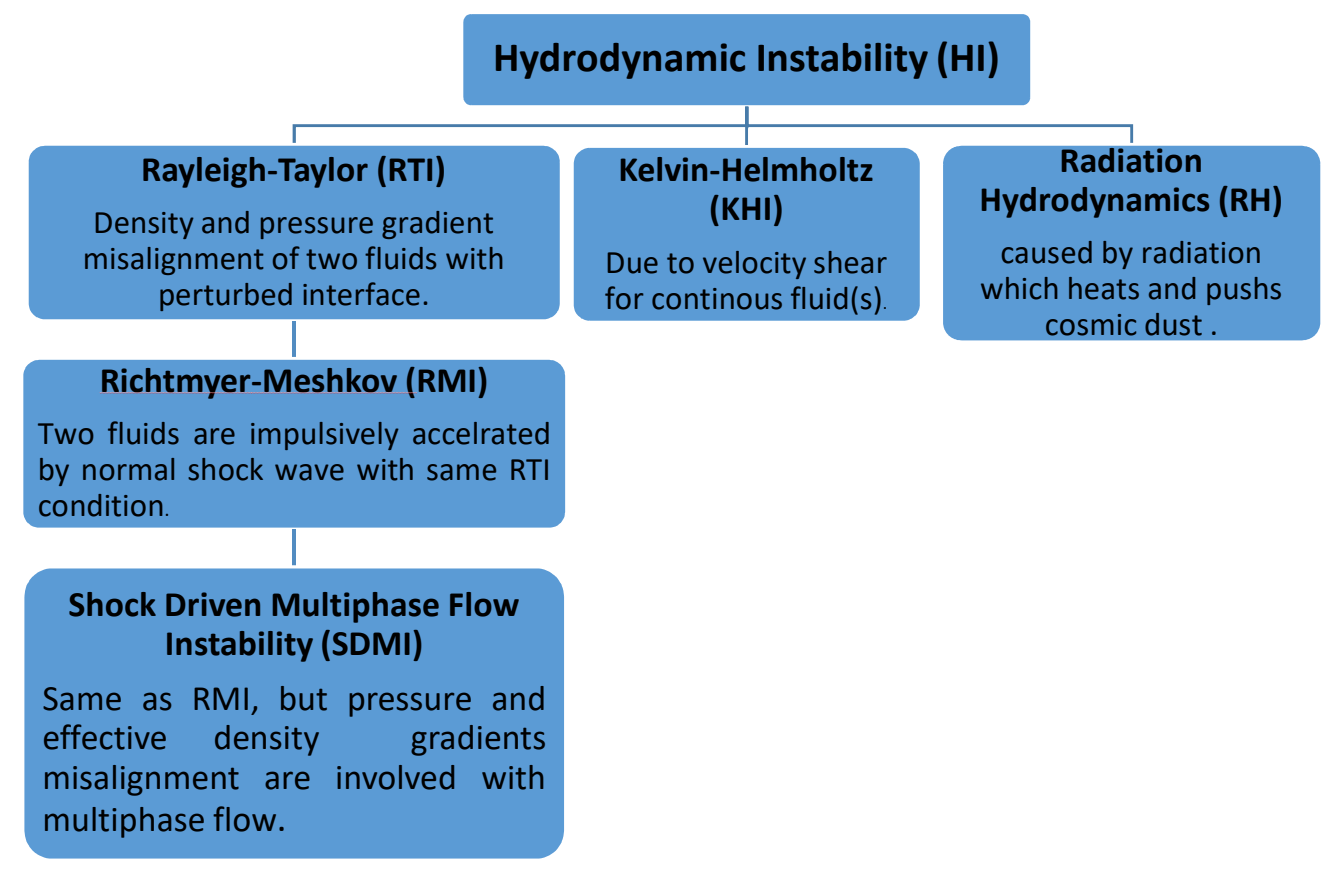

Figure 1.1: Classification of HI's

are both limiting types of RTI because pressure and density gradients with the perturbed interface are required in these two HIs. Finally, the Radiation Hydrodynamic Instability (RHI) plays an active role in planet and star formation. Radiation momentum is negligible on earth, but it does have slight effect on a satellite orbit [3], also, it is very high in the interstellar medium causing different complex HIs. We will list HIs from less to more importance to the current research, starting from Kelvin-Helmholtz Instability (KHI) ending with SDMI.

\subsubsection{Kelvin-Helmholtz Instability (KHI)}

The KHI occurs when a difference in velocity gradients across a fluid interface exists, whether these differences are related to a single continuous fluid or different fluids that flow in parallel. In KHI mechanism, the shear between fluid(s) layers is the main reason causing interface deformation and developing the KHI, see Figure 1.2.

Figure 1.2 shows a simple experiment to initiate and develop the KHI. The top part 
A)

\begin{tabular}{|l|}
\hline Light Fluid \\
\hline
\end{tabular}

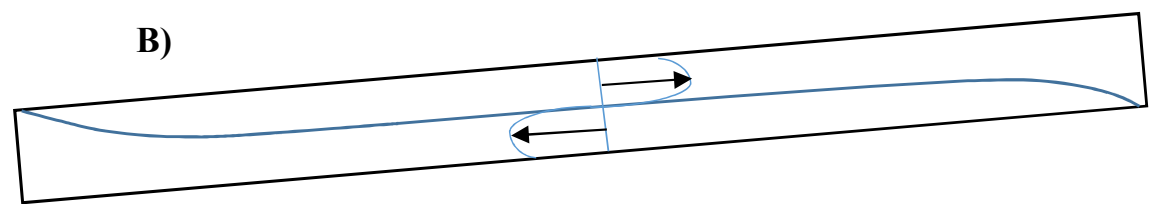

C)

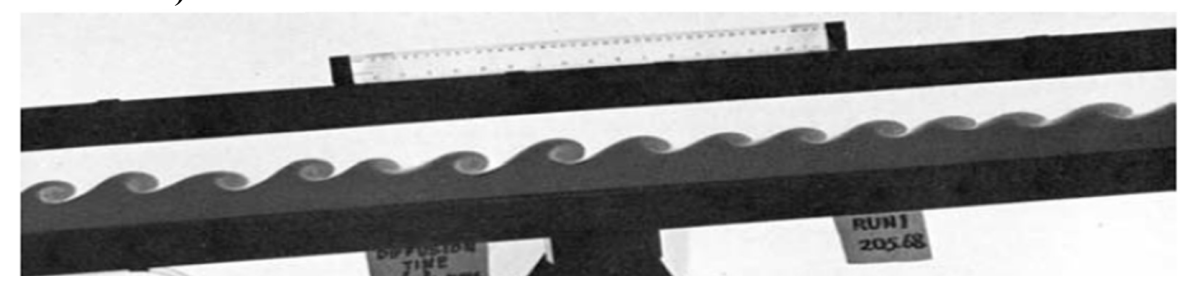

Figure 1.2: Kelvin-Helmholtz instability at the shear interface between two liquids in a tube of rectangular cross-section A) before tilting. B) After tilting shows velocity profile. C) Actual image for B after tilting [4]

of the figure, illustrate a container, which has two different fluids in stable condition, and the interface is straight. If we tilt the container as in part (B), the interface will deform and makes vortex sheets as illustrated in part (C). Vortex generation and deposition on the interface will grow due to shear forces in opposite directions, above and below the interface. We can depict the velocity profile as shown in part (B) of the figure that causes opposite shear stress and then the KHI. Now, let us move to the most significant instability, Rayleigh-Taylor (RTI) that has many applications and limiting cases.

\subsubsection{Rayleigh-Taylor Instability (RTI)}

The Rayleigh-Taylor Instability (RTI) occurs when two fluids with different densities and perturbed interface initially are pushing each other due to the difference in their potential energy. This definition fits with Lord Rayleigh's instability theory (1883), who derived instability model of the interfacial motion of ideal incompressible fluids. In his experiment, when we have two different immiscible fluids (dense fluid supported 


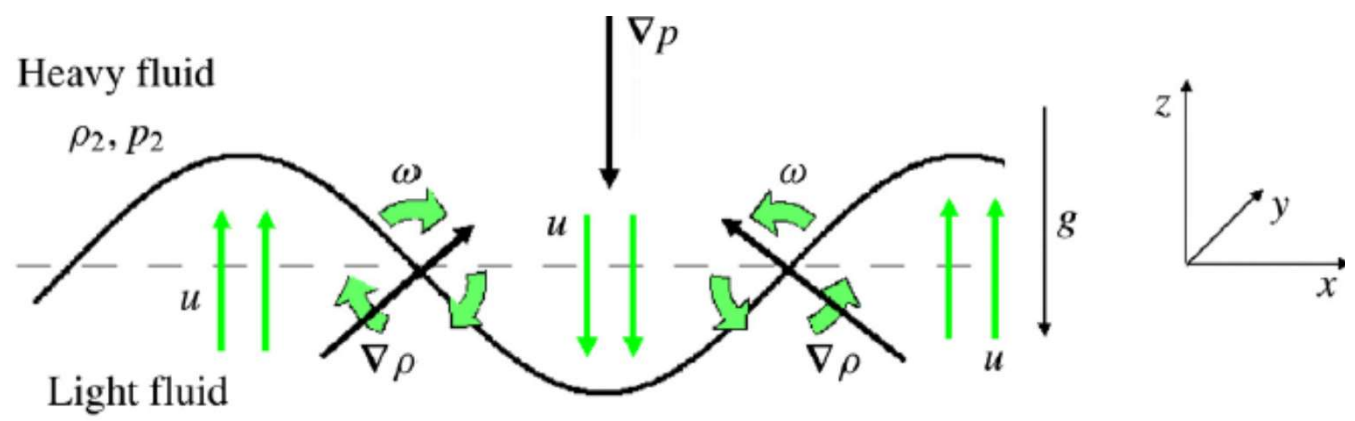

Figure 1.3: unstable RTI configuration, where baroclinc torque create vorticity that empower baroclinicity.

by light fluid) with a perturbed interface, the light fluid penetrates the heavy one due to gravity causing continuous interface deformation [5]. Taylor, however, recognized that interface instability could also initiate when light fluid is accelerated towards dense fluid. In both scenarios, light fluid is penetrating or pushing dense fluid, causing unstable interface. To be more precise, RTI happens when pressure and density gradients are misaligned in fluid flow such that pressure and density gradients vectors in opposing directions $(\nabla \rho . \nabla p<0)$. Misalignments cause what is called baroclinic torque on interfacial region casing instability. However, when pressure and density gradients vectors in same general directions $(\nabla \rho . \nabla p>0)$, heavy fluid is pushing light fluid. In this case, the interface will be stable.

clarify the baroclinic effects on the interface, let us start with momentum Euler equation of motion. By taking the curl, we can find the vorticity equation of fluid motion as follows.

$$
\begin{gathered}
\frac{\partial \rho \mathbf{v}}{\partial t}+\nabla \cdot(\rho \mathbf{v} \mathbf{v})+\nabla p=0 \\
\frac{\partial \omega}{\partial t}+(\mathbf{v} \cdot \nabla) \omega=(\omega \cdot \nabla) \mathbf{v}-(\nabla \cdot \mathbf{v}) \omega+\frac{1}{\rho^{2}} \nabla \rho \times \nabla p
\end{gathered}
$$

The pressure term in the right $\frac{1}{\rho^{2}} \nabla \rho \times \nabla p$ measures how strong the baroclinic torque is. When $\theta$ equals 180 or 0 , this term will be zero because of $\sin (\theta)$ of cross product equals 
zero (pressure and density gradient are parallel). However, when $\theta$ equals 90 the $\sin (\theta)$ of cross product will be one, maximizing baroclinic torque and initiating two rollups (at $\theta$ equals 90 and -90) see Figure 1.3. Figure 1.3 matches Lord Rayleigh experiments and shows the three RTI's ingredients $(\nabla \rho, \nabla p$, and perturbed interface). Vorticity direction can be determined by right hand rule of the cross product $(\nabla \rho \times \nabla p)$. This vorticity will increase the baroclinic effect at interfacial region making unstable RTI.

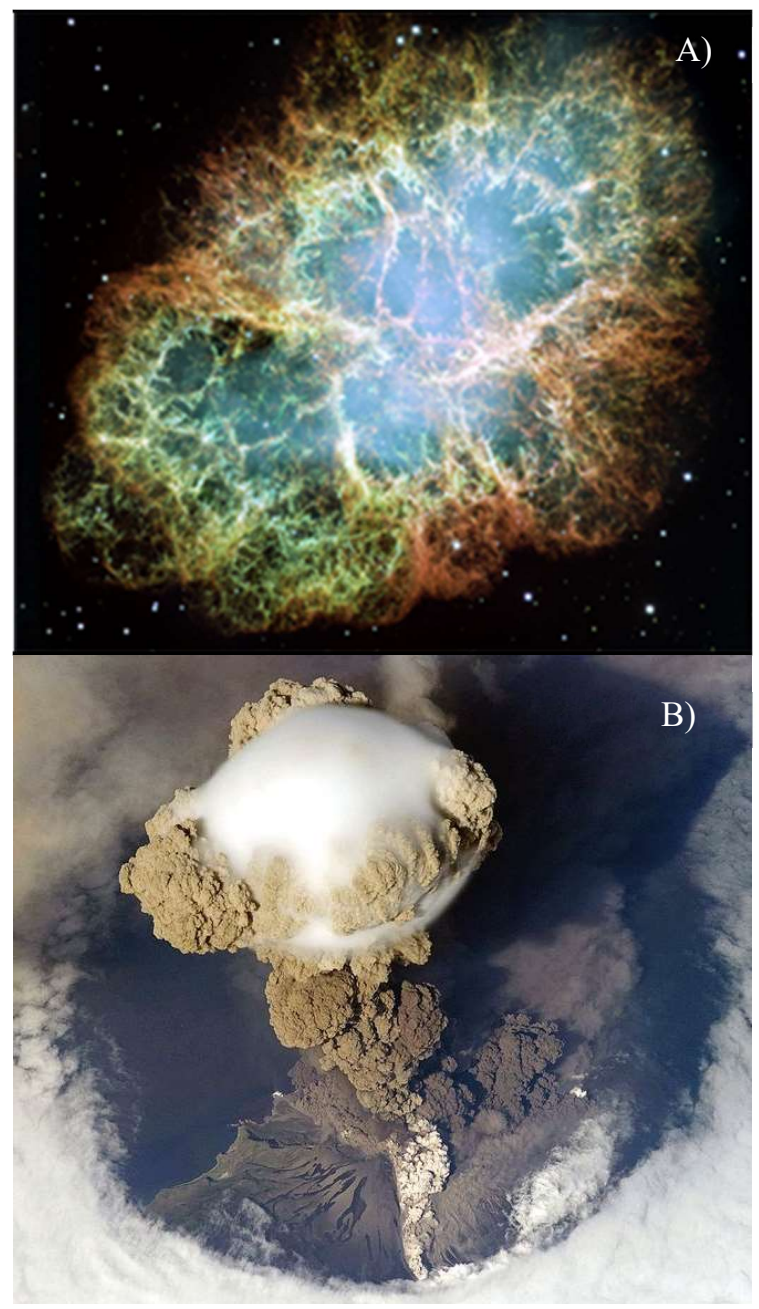

Figure 1.4: RTI, A) Hubble Space image of the Crab Nebula [6],B) Sarychev volcano [7].

Pressure gradients may come from a difference in potential energy, from accelerated fluid flow, or both. Pressure gradients are the driving force in the RTI. The RTI can be seen in large scale phenomena in outer space like crab nebulae when in- 
terstellar gas is pushing the galactic plane by a magnetic field and cosmic rays. We also can see this exciting instability on earth; for example, natural mushrooms clouds from some volcanic eruptions, when hot gases speed up from volcano crater making a column of hot gases, these gases eventually cools off and getting denser interacting with upcoming hot low-density gases. Vortex ring will deposits around gasses column at the heights level that gases can reach, as well as drifting the gases down from the center of the column. See Figure 1.4 A\&B. These two examples do not necessarily have only RTI type. Most natural phenomena have different types of HIs, but when we say this is an RTI or a KHI means that is the most dominant HI. Let us go back to Figure $1.4 \mathrm{~B}$ in a volcanic eruption. The KHI is also involved because different velocities of different gasses are expected around the column of hot gases, and it might develop small scale KHIs, but these small scale HIs are not dominant. Henceforth, when HI type is mentioned in the context, this means it is at least the dominant type of HI. Another example, the interstellar medium has many different instabilities, but the RTI is the primary type. The RTI is an introductory to the Richtmyer-Meshkov Instability (RMI) which is going to be discussed next section.

\subsubsection{Richtmyer-Meshkov Instability (RMI)}

The Richtmyer-Meshkov Instability (RMI) initiates when a shock wave interacts with misaligned pressure and density gradients vectors in fluid flow. The RMI is distinguished from the RTI by impulsive acceleration while the RTI has continuous acceleration, also, it can happen in two different cases, when Heavy fluid is accelerated towards the light fluid or the opposite. However, the RMI is considered a particular case of the RTI due to pressure, and density gradients with the perturbed interface existence. The RMI is significant, and it has wide applications, for instance, inertial confinement fusion (ICF) in which the RMI causes mixing between ablator material and fuel, causing nuclear fusion. Another example is the interaction of a moving 
shock wave within a flame in many combustion engines [8].

\subsubsection{Shock- Driven Multi-Phase Flow Instability (SDMI)}

A special case of RMI is when a gas phase accelerated by a shock wave towards multiphase flow with perturbed interface. Shock- Driven Multi-Phase Flow Instability (SDMI) is generated. In this case, pressure and density gradients will be involved with another phase like solid or liquid particles of dispersed phase. Effective density of interface will be considered to quantify the density change for multiphase region. However, particle size and location distribution will play a significant role in this type of instability. Again, the interaction between particles clouds and the accelerated gas initiates vortex motion and increase flow mixedness. This instability can be seen in scramjet and pulse detonation when liquid fuel is mixed with air. SDMI is observed in interstellar medium (ISM), when radiation and hot gasses are pushing dust out word forming different types of HIs.

The next section will go through SDMI with radiation, which is is common in circumstellar medium (CSM).

\subsubsection{Shock- Driven Multi-Phase Flow with Radiation Insta- bility}

Radiation Hydrodynamics is a part of hydrodynamics that studies the interaction of radiation with matter. Radiation has profound effects on forming celestial objects in the very long term. It drives the gas, dust, and even big celestial objects. Gravity and radiation are playing a significant rule in developing all types of hydrodynamic instabilities at interstellar and circumstellar medium [3].

All types of hydrodynamic instabilities are expected to be seen at circumstellar medium (CSM), and interstellar medium (ISM), see Figure 1.5.This figure shows long 
term evolution of matter near Asymptotic giant branch (AGB) star with the signature of KHI and RTI visible with mushroom-like signatures. AGB star is an exciting phase that attracts many astronomers to observe and study its evolution before the star goes to the white dwarf phase. In this phase, the star starts to expel matter triggered by pulsating motion look like a heart beating and combined with the shock waves. In this phase, the star starts to expel matter (Gas+dust) triggered by pulsating motion look like a heart beating combined with a shock wave. This phenomenon is puzzling because many factors can control the morphologies of the developed instabilities. To better understand this, I will quote some important statements that I relied on in my research relating to the SDMI evolution in CSM and ISM.

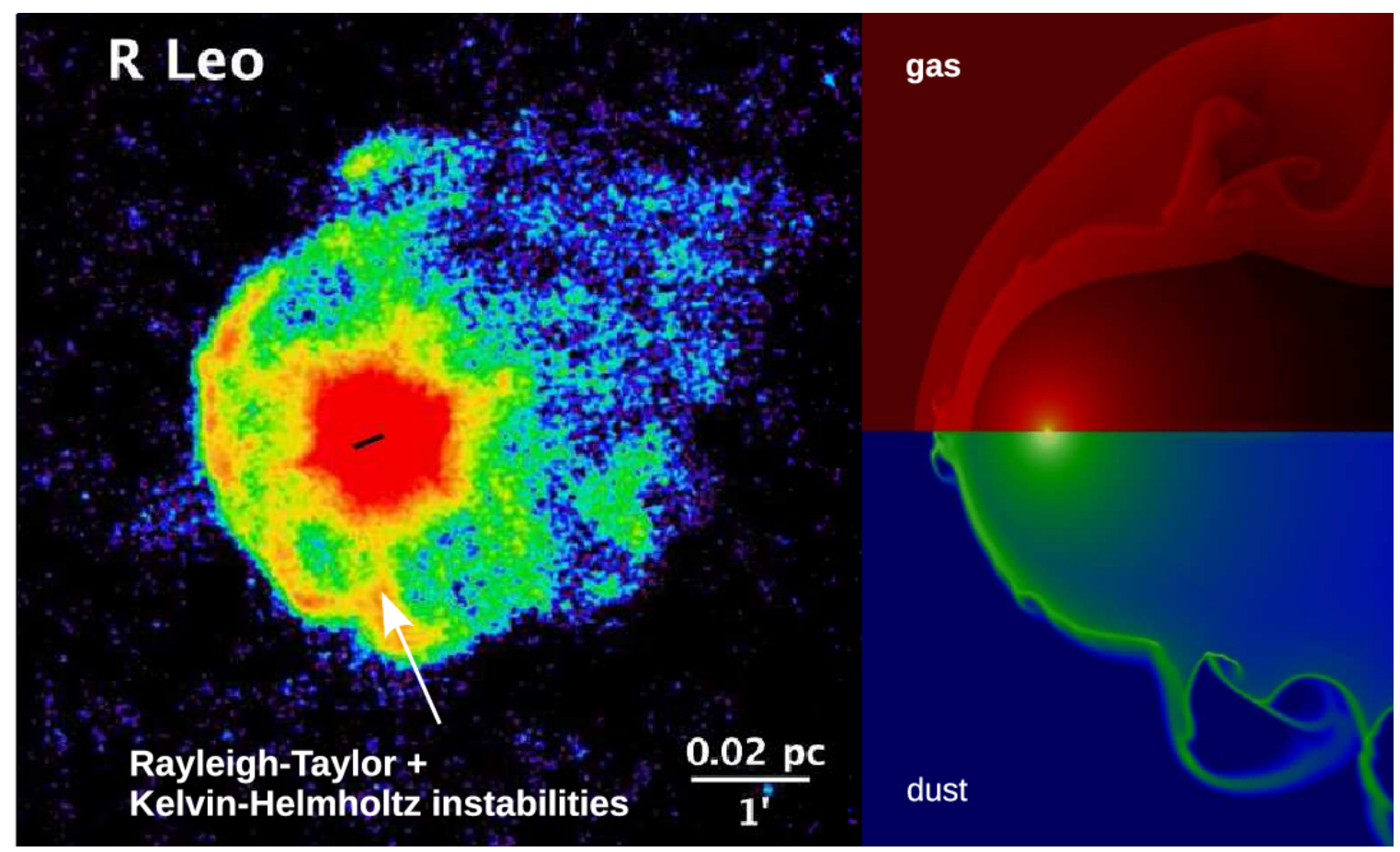

Figure 1.5: R Leo (left) compared to a hydrodynamical simulation of the wind-ISM interaction computed with the AMRVAC code [9] The parameters for this simulation are a stellar wind velocity of $15[\mathrm{~km} / \mathrm{s}]$, a constant gas mass-loss rate of $1 \times 10^{-6} M_{o} / \mathrm{yr}$, a dust-to-gas mass ratio of 0.01 , a space velocity of $25[\mathrm{~km} / \mathrm{s}]$, a local ISM density of $2 \mathrm{~cm}^{-3}$, and an ISM temperature of $3 \mathrm{~K}$. The upper right figure shows the gas density, which range (in log-scale) between $10^{-24}$ and $10^{-19}\left[\mathrm{~g} / \mathrm{cm}^{3}\right]$; the lower right figure represents the dust grain particle density, ranging (in log-scale) between $10^{-10}$ and $10^{-3.5} \mathrm{~cm}^{-3}[10]$ 
Woitke stated:

"Shock waves created by the stellar pulsation have been identified to trigger the process of dust formation close to the star, and radiation pressure on newly formed dust grains can overcome local gravity"[11].Peter Woitke

, Höfner statement was:

"The basic scenario for this process is that of a pulsation-enhanced dustdriven wind, stellar pulsation triggers propagating atmospheric shock waves which intermittently lift gas above the stellar surface, creating dense, cool layers where solid particles may form"[12],Susanne Höfner

, Bladh stated:

"Stellar winds observed in asymptotic giant branch (AGB) stars are usually attributed to a combination of stellar pulsations and radiation pressure on dust. Shock waves triggered by pulsations propagate through the atmosphere, compressing the gas and lifting it to cooler regions which creates favourable conditions for grain growth" [13],S. Bladh

In fact, there is a debate about the driving mechanisms of the stellar winds. There are two possible driving mechanisms, Radiation-driven winds, and Pressure-driven winds. For Radiation-driven winds, dust and gas molecules absorb the radiationinduced from plasma (wind of hot gases) and get acceleration towards the ISM; However, in the Pressure-driven winds, mechanical motion is provided by convection zone, stellar pulsation, shock wave, etc. A good example of the Pressure-driven winds is the solar wind, which is generated by the dissipation of the mechanical energy originating from the solar convection zone[14].

In this research, I choose the Pressure-driven winds scenario for its simplicity and matching with our SDMI model. In the next section, we will go through the mechanisms of the SDMIs shock tube facility and the interstellar medium as well. 


\subsection{Mechanism of SDMI}

In the previous sections, we have mentioned explicitly three important shared requirements to develop the RTI and RMI (density, pressure gradients and a perturbed interface). Similarly, the SDMI has the same conditions in macroscopic level; however in microscopic point of view there are some differences. There is no distinct interface in microscopic level in SDMI because each droplet or say the particle will have its own interface, but we can consider an effective density of dispersed phase macroscopically to quantify the density differences between downstream condition and seeded average conditions. The density gradients form from particle clouds instead. To depict the SDMI, let us make comparisons between mechanisms of RMI and SDMI such that the mechanisms of SDMI will be clear. Figure 1.6 A shows RMI mechanisms. When moving shock wave travels inside shock tube subjected to downstream flow condition $\left(\rho_{1}, p, T\right)$ from left to right and hits another fluid with $\left(\rho_{2}, p, T\right)$ that has a specific interface geometry, say circular shape, a shock front and post shock condition will interact with the interface as shown below, causing interface distortion, and a vortex motion will be created due to the misalignment of the pressure and density gradients. Misalignment will create a variable baroclinic torque that has a maximum value at $\theta=90^{\circ}$ and a minimum value when $\theta$ equals zero or $180^{\circ}$ because the pressure and density gradients vectors are parallel. This torque is the reason behind rollups that we see in the top and bottom points at the interface. Two different scenarios are expected in these cases depending on the pressure and density gradients alignment orientation. The rollup is clockwise on the top and counterclockwise at the bottom $\left(\rho_{2}>\rho_{1}\right)$; second, the rollup is reversed on the top and the bottom of interface if $\left(\rho_{1}>\rho_{2}\right)$, see Figure 1.6 B.

Now, for SDMI the interface will be subjected to the same shock tube condition say $\left(\rho_{1}, p, T\right)$ but it will be seeded with particles or droplets with higher density than the pre-shock conditions. For simplicity, let us consider solid particles cloud with 
A)

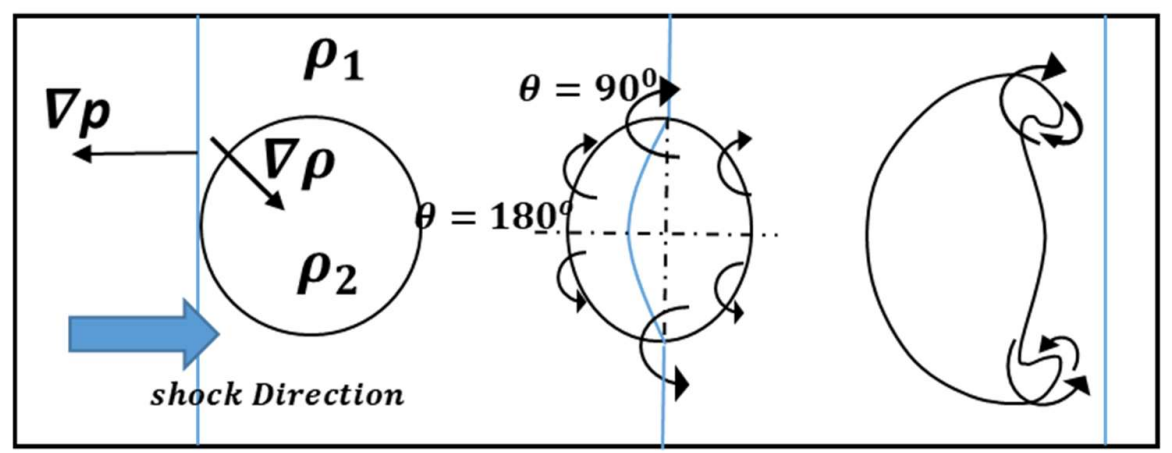

B)

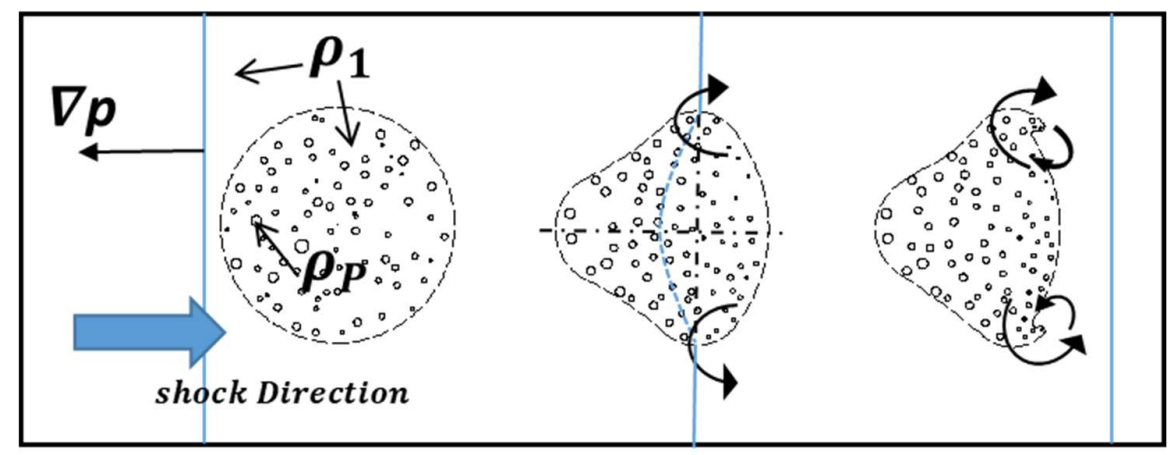

Figure 1.6: RMI production, density gradient vector inward, B) SDMI production, effective density gradient vector inward, particles lag behind.

specific size and local distribution. In this case it is hard to distinguish the interface in microscopic level but the interface shown in the figure 1.6B is meant to be a virtual interface that surround all particles cloud. Particles responses to the incident shock will be different relying on their properties, but general speaking they will lag behind unseeded flow that means there is a relative velocity between seeded region and fluid flow. "Lagging behind" comes from the drag forces of particles cloud against impulsive flow.

To be more precise, velocity change across virtual interface will have similar effect to the baroclinic torque subjected interface in RMI mechanism, also, seeding concentrations are significant in SDMI production and evolutions. Figure 1.6B shows different response of particles relying on their sizes; for example, large particles lag behind unseeded flow causing tail-like shape. However small particles have less lag or 
say behave like flow tracer compared to the big particles. It is hard to tell the whole story of particles behavior against incident shock because particles might merge, break up, evaporate or rotate on themselves. To sum all above, SDMI has similar evolutions to the RMI when particles are smaller, but it deviates from RMI as particles are bigger and the physics in this case will be more complex than small particles case. Particles properties (size, location, velocity, distributions, etc.) are the main factors that control SDMI instability.

\subsection{Mechanism of SDMI at circumstellar medium}

The hydrodynamic instability is more complicated at circumstellar and interstellar medium. The complications come from having different mediums with different physics.A typical sketch that shows all these envelops and complications is shown in Figure1.7. This figure shows different envelops with the radial position from the center of the star all the way to the ISM.

The mass loss mechanisms are still not understood well yet but again relying on the second scenario we mentioned in section 1.1.5. Condensation of high dense gases to make dust due to shock waves generated at convection stellar atmosphere, and then the dust generated at this envelope will be subjected to the Radiation and pressure waves which overcome the gravity force. Radiation will push the dust (particles) and the gas at same time and then if particles faster than gas, it will pull the gas along with and vise versa.As we cross the CSM in the space the effects of wind and gravity acceleration decrease, while the effect of radiation is dominant then. SDMI is expected to be in all CSM envelops shown in the Figure 1.7, while SDMIs evolution less at ISM when the the shocks are dissipated. In the ISM, another types will be developed due to the radiation effects like KHI and RTI as we saw in figure1.5. 


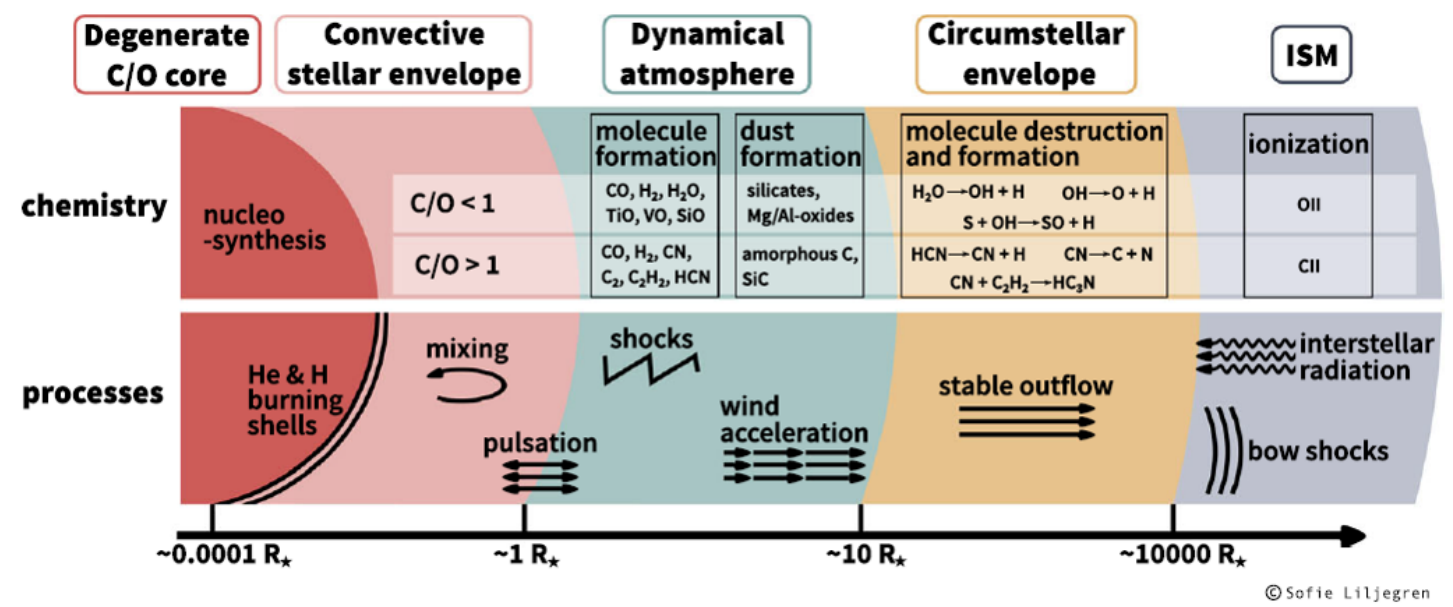

Figure 1.7: Schematic drawing of an AGB star. Pulsations and dust formation are the two key ingredients for the generation of a stellar wind, with typical terminal velocities between $10-30[\mathrm{~km} / \mathrm{s}]$ and mass-loss rates between $10^{-8}$ and $10^{-4}\left[M_{o} / \mathrm{yr}\right]$. Several chemical processes are shown at the typical temperature and radial distance from the star where they occur. The penetration of interstellar ultraviolet photons in the outer envelope is shown as well. Understanding the chemical processes in AGBs is crucial to know the chemical content of the (unprocessed)ISM[10][15].

\subsection{Challenges and Goals of Current Research}

In this research, since we are trying to validate our code first and extend code capabilities to simulate the hydrodynamics of circumstellar medium, I will be listing the challenges for SDMI in shock tube first,then interstellar medium as follows.

1. High speed flow around $300[\mathrm{~m} / \mathrm{s}]$ with moving shock wave (Mach equals 1.67 ), and short time of interface evolution $\sim 5[\mathrm{~ms}]$.

2. Particles size and local distributions have random nature so that choosing distribution parameters should be carefully done.

3. We have to have a good numerical scheme that can catch most dominant physics, as well as a good imaging system to monitor SDMI's evolution.

The main goal of first part of the research after solving the above challenges is 
to verify that implemented Multiphase particle in cell method (MP-PIC) in FLASH (open source hydrodynamics software) can give close comparisons between experimental measurements and numerical solutions. If experimental results matches the numerical results well, we will apply SDMI on interstellar medium by considering Radiation pressure force on both on (dust) particles and gas flow (star wind).

the challenges related to the second part of research are:

- Large scale of interstellar medium $>4 \times 10^{15} \mathrm{~m}$ this require high performance computing memory from $10^{5}$ to $10^{7}$ CPUs.

- Three important envelops near AGB star have three different physics with specific transition regions at interfaces among all zones.

- There is a debate about the mechanisms by which AGB star phase near convection envelop develops.

- Pulsating,shock-waves, chemical reactions,phase change, multi-species, nuclear fusion, plasma with extreme radiation are all different processes that can be considered at CSM.

- It is hard to give appropriate conditions to the star due to the complex scene at convection envelop, so it requires careful assumption to give reasonable simulation because of the big effects of the star to the whole problem domain.

our goal in the second part of the research is to simplify the above challenges and implement new drag model at rarefaction condition,implement gravity model,and Radiation model. 


\subsection{Accomplished Tasks in This Research}

The first task of the current study is done by comparing experimental and numerical solutions of interface evolutions on shock tube facility in University of Missouri at Columbia, which are done by considering the following aspects.

1. Simulating a shock tube test section only rather than having a whole shock tube size because of the large computational domain $(8.43[\mathrm{~m}]$ long and $13.97[\mathrm{~cm}]$ width) and choosing computational size equals to $2[\mathrm{~m}]$ long and $3[\mathrm{~cm}]$ width.

2. Initialize the physical conditions of shock tube that are close enough to experiments conditions to insure that we have started from same point of solution.

Early results shows matching between experimental results done in shock tube facility and numerical results of FLASH simulation.

For the second task,I extended the code capability as follows.

- New drag model built on kinetic theory on particles nearly free molecular flow.

- Considering point mass gravity model for both gas and particles.

- Implement Simple Radiation model on both particles and gas. 


\section{Chapter 2}

\section{Literature Review}

\subsection{Background}

Different approaches are used to simulate multiphase flow in computational fluid dynamics. However, each type of multiphase flow needs a specific implementation. For instance, if we have two different immiscible fluids (liquid and gas phases) with surface tension at the interface, we can use the volume of fluid method (VOF) to solve this interaction. This method is an Eulerian representation for both phases. Another example, let us assume dispersed liquid phase flows within the gas phase. This kind of flow is called particle flow or particle cloud flow, which has a wide range of applications starting from fluidized-bed, spray coating, fire suppression and control ending with pulverized coal, solid-propellant rocket, and abrasive water jet cutter. Different methods of interaction are expected between gas and particle phase in these types of multiphase flow [16]. One, two, and four ways of coupling are expected between particles and gas flow. When particles are driven by fluid flow, particles are considered as a flow tracer; also, this type is considered one-way coupling. Twoway coupling, on the contrary, considers the effect of particles on the flow as well. 


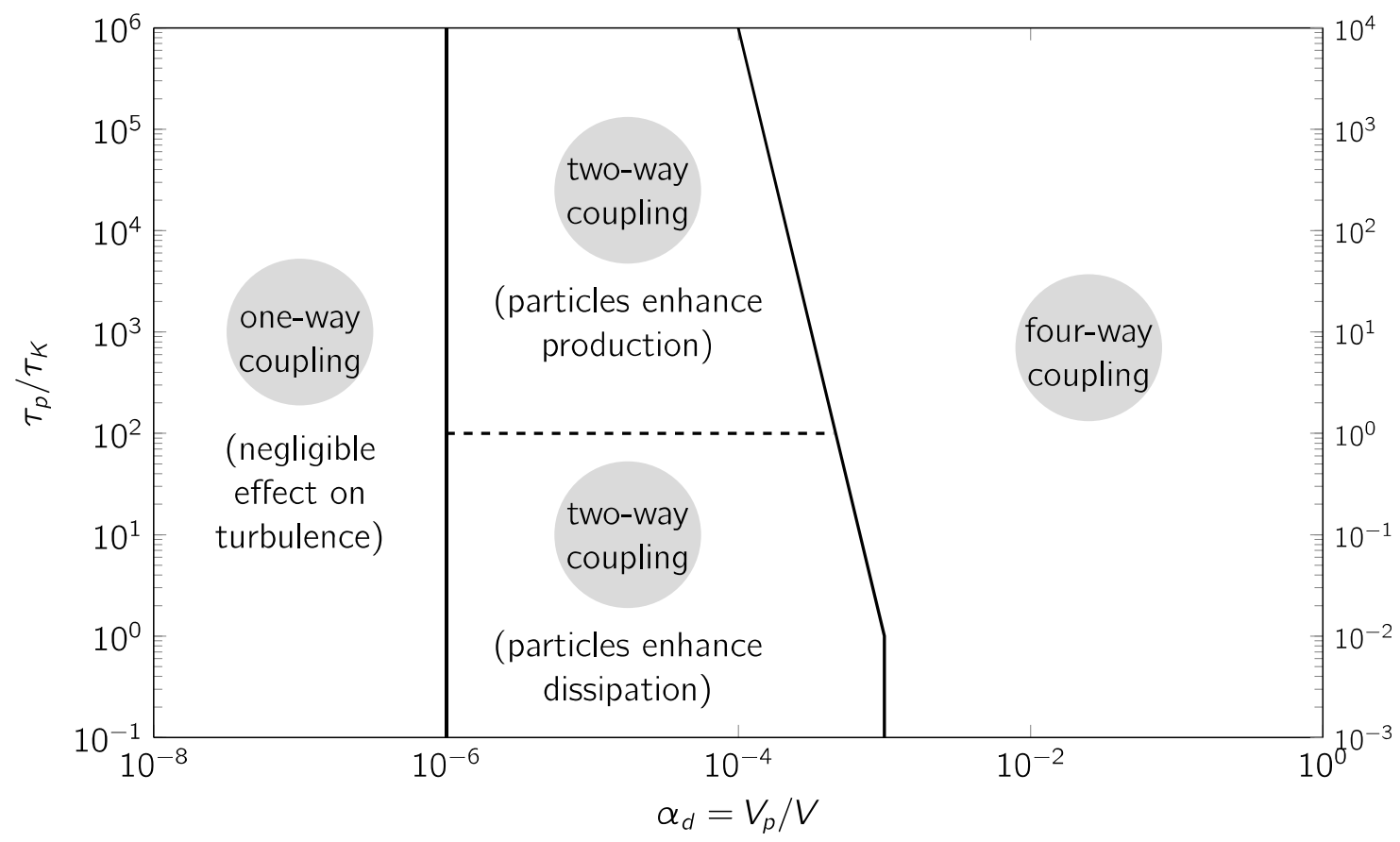

Figure 2.1: Classification of phase-coupling mechanisms according to Elghobashi [17].

Lastly, the four-way coupling considers flow to particles and particles to particles interactions as well, see Figure 2.1 that shows all different couplings as a function to particles volume fraction.

The solution of these interactions can be solved by Eulerian - Eulerian model (E-E) or Eulerian-Lagrangian (E-L) model. E-L simulation tells more information about the dispersed phase; also, each particle will have its equation of motion and its computational point. This method (E-L) constrains and limits the number of particles we can deal with in a specific problem; as a result, we have three different approaches to deal with particle cloud problems according to the number of particles. So, if we have a smaller number of particles per volume the particles cloud is considered as dilute and the best model would be E-L because the particles flow coupling is one or two-way coupling and particles are not dense enough to consider them as a continuous phase, but if the particle cloud is dense then E-E is advantageous due to the continuity of particles. E-L can be divided into discrete element method (DEM) when we have 


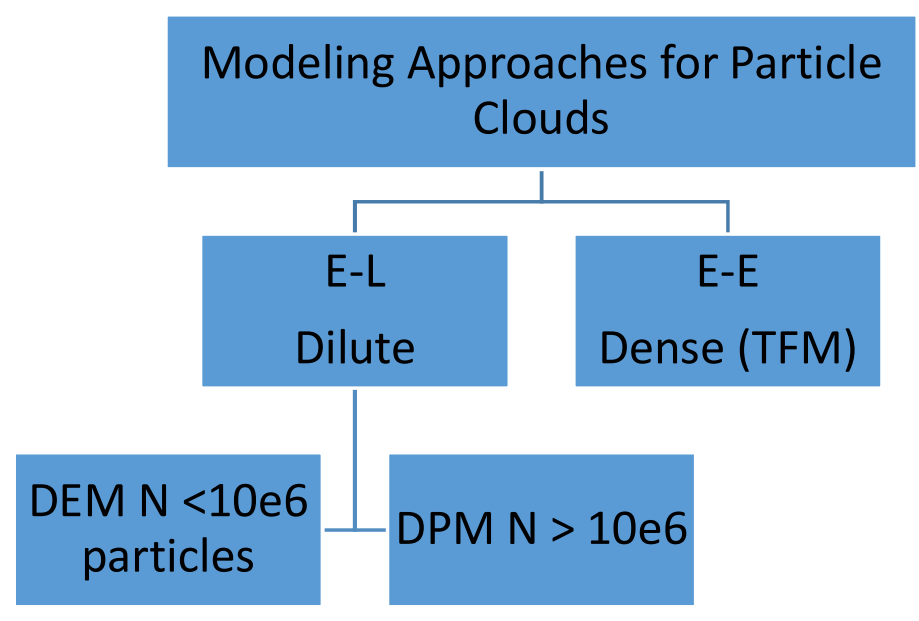

Figure 2.2: classification of particles cloud approaches[16].

a limited total number of particles (solver dependent number) and Discrete Parcel Method (DPM) when we have many millions of particles that a computer cannot handle, Discrete Parcel Method (DPM) assumes that each parcel has many particles with the same properties (location, size, and velocity). Please take a look at the hierarchical chart, see Figure2.2. In the SDMI problem we are dealing with it is considered as dilute because the volume fraction of particles less than 0.001. We will discuss this later in chapter four.

\subsection{Multi-phase Particles in Cell Method (MP- PIC)}

The multiphase particles in cell method (MP-PIC) has been developed by Andrews O'Rourke. This method maps Lagrangian particles to and from Eulerian computational domain (mesh), and it can handle a wide range of dispersed phase from dilute to dense flow. O'Rourke's model was a one-dimensional simulation that deals with vital fluidized-bed application like combustion and catalytic cracking, and so on [18].The MP-PIC has many advantages that make it desired for an extensive scale of particles flow interaction simulations[19].MP-PIC method was developed further by Snider to 
solve two- and three-dimensional gas-particle interactions at which interpolation operators and their parameters are conservative and give a fast solution for large particles populations [20][21].MP-PIC method is used in different software and open-source codes. McFarland et al., 2016 [22] use multi-phase particle-gas interaction with RMI in arbitrary Lagrange Eulerian code (Ares), they highlighted on many parameters like incident shock strength, effective density difference, and multiple time relaxation effects.Dahal, and McFarland extended this work using MP-PIC with hydrodynamic code in FLASH to simulate shock driven multiphase flow. A simple validation was done by comparing velocity and temperature histories with analytical models[23].

The MP-PIC method is currently used in Computational particle fluid dynamics software (CPDF) [24]. OpenFOAM (open source code uses $\mathrm{C}++$ libraries) also has many solvers that use MP-PIC[25]. In this research, we used the implemented MP-PIC method in FLASH, open-source code developed at the FLASH center at the University of Chicago 1997[26]. FLASH has many units that are easy to access, update, and develop. It is a modular, adaptive, parallel, and multi-physics simulation code that handles compressible fluid flow efficiently. Compressible fluid flow is implemented in the hydrodynamics portion of code that uses piecewise parabolic (PPM) and other like Weighted Essentially Non-Oscillatory (WENO) methods as a discretization method for gas flow[26]. Adaptive Mesh Refinement (AMR) techniques are used to cover large dynamic ranges of problem scales. it uses the PARAMESH package, incorporated into FLASH, for AMR, providing a block-structured mesh. The resolution is increased for areas of greater interest, by a factor of 2 , in each direction, for each level of refinement. For more details on FLASH, please consult Flash Center[26]. 


\section{$2.3 \quad$ Experimental Studies}

The Shock-Driven Multi-Phase Flow Instability (SDMI), which we are dealing with, is a combination of multi-phase particulate flow and Richtmyer-Meshkov (RMI) Instabilities. To prepare SDMI experments, we need a shock tube facility to provide an impulsive acceleration flow, also, seeded particle flow inside a shock tube with a specific perturbation. Lastly, we must have a suitable imaging system to capture the evolution of the dispersed phase.

J. W. Jacobs et al.,2013, used vertical shock tube to perform RMI with threedimensional random perturbation, a vertical oscillation of gas column is created within shock tube to produce faraday waves at interface and gas flow is impulsively accelerated with Mach 1.2. A high-speed camera is used to capture a sequence of images using Mie scattering[27].Balakumar et al. studied turbulent mixing in RMI under single incident shockwave with Mach equal 1.21 followed by reshock wave with Mach 1.14, measurements of velocity-density show nonlinear growth of interface without transition to the turbulence for incident shock, but the turbulence mixing is developed in reflected shock-wave[28].

Many scientists have addressed RMI studies experimentally and numerically[29][30]. The first Author who considers different densities in RMI related to another phase was Vorobief; he seeded liquid particles as a heavier phase in a shock tube to create a SDMI which has similar results to RMI[31].Vorobief et al., 2015 has made experimental, and numerical comparisons for RMI with seeded particles, careful choosing to the initial condition using FLUENT is performed for Mach number from 1.2 to 2.1[32]. John B. Middlebrooks et al.[33], has conducted experimental studies for the incident shock and reshock in acceleration with water particles for different Atwood numbers. The interface is shocked by Mach 1.67. Results show particle evaporation and mixing increases after re-shock condition.

In the first part of the present study, we used the FLASH code, to validate the 
SDMI evolutions in the simulation to confirm that the implemented code is able to deal with particulate flow problems under accelerated flow. This can be done when the matching is accomplished between numerical and experimental results. The vital part of this part of the research is initializing the problem which is sensitive to the Lab conditions to compare with experimental image taken by the lab imaging system.

\subsection{Hydrodynamic Simulations near AGB Stars}

Stellar winds are common phenomenon for all stars. Also, it is the main reason of hydrodynamics instabilities developed in circumstellar medium (CSM) and interstellar medium (ISM). For example, sun has mass loss rate $\left(\dot{M}=2 \times 10^{-14} M_{o} / y r\right)$, Asymptotic Giant Branch (AGB) stars have highest injection rate compared with the other stars $\left(\dot{M}=10^{-4}-10^{-8} M_{o} / y r\right)[34]$.

Authors who simulate AGB star addressed CSM, ISM, and the star core in different levels of sophistication. Woitke, Peter, 2006, solved gas equations of motions with the time-dependent formation of dust from gas with a radiative transfer equation. Woitke didn't consider shock wave or pulsating effects in his model. However, radiation model is solved by considering Monte Carlo method for scattering and reemission. His model would fit with the ISM envelope.B. Freytag, and S.Höfner,used different hydrodynamics code to produce 3D model and showed that large convection cells and pulsation give spherical expansion of shockwaves and leviating materials into cool region to allow dust formation, the convection cells in the simulation are so large such that the shocks appear to be spherical justifying the the spherical symmetry [35]. Höfner, et al,2017, solve a 3D Hydrodynamics model of AGB star with effects of convection and radial pulsation on atmospheric structure using CO5BOLD code.His results shows that convection, waves, and shocks all make dynamic pressure to push material to the dust form layer where temperatures are low enough and gas densities 
are high to allow condensation[36].

The Author here adopts the same idea of developing convection cells ejected from the star itself by imposing specific conditions that generate particular types of shocks with specific shape.we will address this in the results section. 


\section{Chapter 3}

\section{Theory and Governing Equations}

\subsection{Background}

Investigating SDMIs in two different problems require all critical parameters and dimensionless numbers we are dealing with. For example, in shock tube problems, we have to deal with the compressible fluid,so shock speed, Mach Number $M_{a}$, Atwood Number $A_{t}$, etc.SDMIs near AGB stars, however, need more parameters to include, like Knudson Number $K_{n}$, Radiation Intensity $(I)$, Gravity $(g)$. So, to understand these inputs well, we will go through all these parameters and dimensionless number through this chapter.

At the end of this chapter, we will describe the governing equations and solution method for both problems. Generally, our systems of equations will be solved using a finite volume technique using the piecewise parabolic method (PPM) for Eulerian gas equations,and Particles in cell method for Lagrangian systems of particles cloud.

We mentioned earlier three critical ingredients. Those are needed to develop RMI, which are pressure gradient, density gradient, and misalignment between pressure and density gradient vectors or say perturbed interface between two different densities. 
These requirements also needed for SDMI with two differences, as discussed in chapter one. Pressure and effective density difference across the imaginary interface are mandatory to cause an SDMI. To be more precise, our SDMI in the first problem is also combined with RMI due to a slight variation in the density between the carrier gas and surroundings. Before we quantify these parameters, we have to consider them as our inputs to the shock tube experimentally and numerically. The following sections will go through these parameters.

\subsubsection{Mach Number $M_{a}$}

Mach number is a dimensionless number which represents the ratio of flow speed to the speed of sound in that medium, Mach number has a direct relation to the pressure drop across a moving or a standing normal shock wave. In the case of an ideal gas, the relation of pressure ratio as a function to the Mach number is as follow [37].

$$
\frac{p_{o}}{p_{r}}=1+\frac{2 \gamma}{\gamma+1}\left(M_{a}^{2}-1\right)
$$

Where $p_{o}$, pressure at post-shock condition, and $p_{r}$ the pressure at the preshock condition and $M_{a}$ is the Mach number of the moving shock. Equation3.1 does not necessarily represent the relation applied in the shock tube; however, it refers to the fact that Mach number reflects the pressure ratio across the shock wave. In unsteady shock tube relations, all variables are preferred to be as a function to pressure ratio of the driver to driven section at initial time. As a result, giving Mach number or pressure ratio in the shock tube will yield the pressure gradient needed for Hydrodynamic instability.

Mach number is an important parameter to calculate drag coefficients in the com-

pressible flow. It also related to the calculation of Knudson number $K_{n}$. We will go through that in the next sections. 


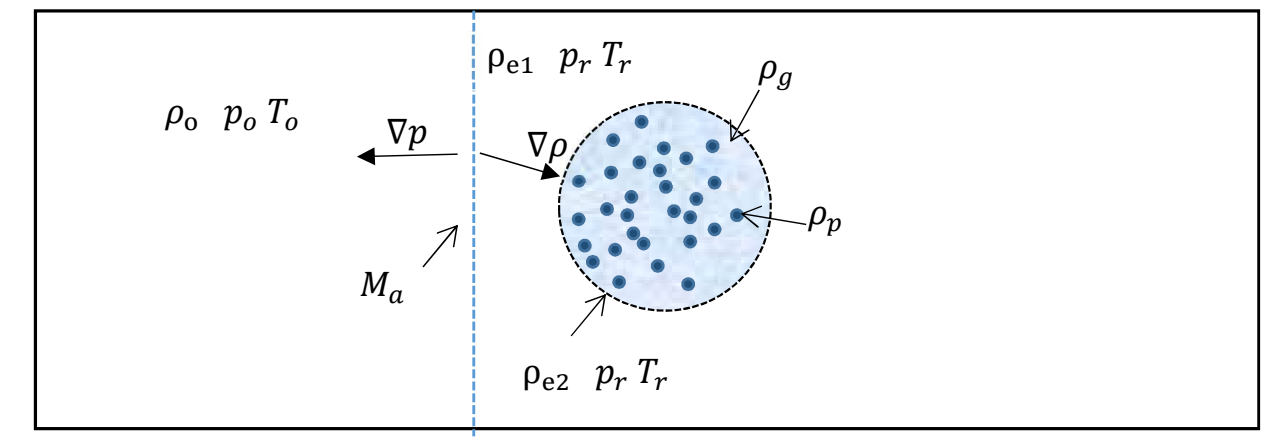

Figure 3.1: Visual representation of the required parameters for the SDMI.

\subsubsection{Effective Atwood Number $\left(A_{e}\right)$}

The Atwood number is the most important parameter that measures density change across interface between two different fluids. It is the ratio of effective density difference to the effective density sum of two fluids. By this definition, $A_{e}$ is as follow.

$$
A_{e}=\frac{\rho_{e 2}-\rho_{e 1}}{\rho_{e 2}+\rho_{e 1}}
$$

Where $\rho_{2}$ effective density of heaver fluid,and $\rho_{1}$ density of lighter fluid, we have changed the densities to an effective density because we are dealing with multi-phase flow (dispersed phase gas+particles) also the carrier gas might be a mixture of different gases. The effective density of the particles cloud is calculated using volume fraction of particles and gas as follow.

$$
\rho_{e 2}=\rho_{p} \alpha+\rho_{g} \epsilon
$$

Where $\rho_{p}, \rho_{g}$ densities of particles and gas of second phase respectively, $\alpha, \epsilon$ volume fraction of particle and gas of dispersed phase respectively, see (Crow,2011)[16]. Figure 3.3 shows densities and parameters needed in SDMI. Calculation of particles mass and volume requires their distributions. We will talk about particles distribu- 
A)

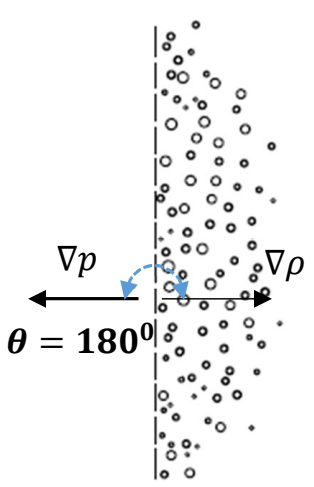

B)

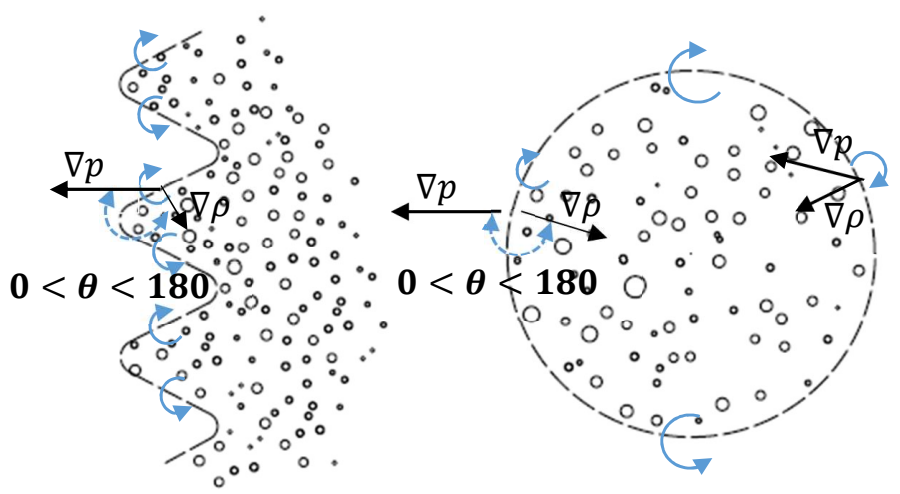

Figure 3.2: Different possible perturbations. A) Straight line interface less instability. B) Corrugated interface high instability. C) Complex Interface with high instability.

tions parameters like mean and standard deviation in chapter four.

\subsubsection{Perturbation of Interface}

The geometrical shape of the interface has an active role in initiating HI. The interface provides the misalignment between pressure and density gradient vectors. Different interfaces can be created when we seed particles inside a shock tube, see Figure 3.2, which shows different possible scenarios. Figure 3.2A shows a plane interface at which the angle between pressure and density gradients equals $\left(180^{\circ}\right)$. This angle is seen from the macroscopic level. In microscopic level; however, each particle has its own interface, it is not necessarily mean there is no instability initiated. However, we can say it will not deposit vorticity faster than case B and C in same figure.

Figure 3.2 B shows corrugated interface, which has sinusoidal interface. This interface is self-developing and empowers interface deformation with time. Figure $3.2 \mathrm{C}$ is complex interface and we can simplify its surface to two semi-circles. There is a difference in alignment on both circular interface sides as shown because once the shock goes through the interface, It will deform making crescent-like shape which is changing pressure gradient vector direction and increasing misalignment between 
pressure and density.Two scenario of interfaces shape are addressed in my research, plane interface (Figure 3.2A) which is adopted in the SDMI initiated close to CSM of an AGB star. Second, complex interface (Figure3.2C) is considered in the SDMI developed in shock tube problem.

\subsubsection{Reynolds number $(R e)$}

Reynolds number is a dimensionless quantity in fluid flow that predict flow pattern. Besides, It is the ratio of inertial force to the viscous force within the boundary layer. The boundary layer might be near a wall or any object like a sphere or even particles [38]. Since we are dealing with moving particles, the Reynolds number can be defined as follow.

$$
R e=\frac{\rho_{g} v_{p g} d}{\mu_{g}}
$$

Where $v_{p g}$ is the magnitude of the particle velocity relative to the gas, $\mu_{g}$ gas kinematic viscosity, $\rho_{g}$ carrier gas density, and $d$ is the particle diameter. Reynolds number is used as scale factor in dimensionless analysis,in addition,it is related to Knudson number see section 3.1.5.

\subsubsection{Knudson Number $\left(K_{n}\right)$}

It is clear that the Mach number refers to the compressibility of flow, and the Reynolds

number reflects the inertia to flow. However, Knudson number $\left(K_{n}\right)$ asses rarefaction effects. Mathematically, it is the ratio of the mean free path of gas molecules to the characteristic length of the addressed scale.

$$
K_{n}=\frac{\lambda}{d}
$$


Where $\lambda$ is the mean free path, and $d$ is particle diameter. We can rewrite this equation in terms of Reynolds Number Re, and Mach number $M_{a}$ as follows[39].

$$
K_{n}=\sqrt{\frac{2 \gamma}{\pi}} \frac{M_{a}}{R_{e}}
$$

It is imperative to classify the flow relying on Knudson's number. In general, if $K n<0.001$, the flow is a continuum, and the fluid flow can be accurately modeled by using Navier stokes equations. While, if $K n>0.1$, the flow is not continuum any more, and then the solution of fluid flow done by using a numerical solution to the Lattice Boltzmann methods (LBM) and inter molecular forces has no negligible effects[39]. In the present study, Knudson's number has an essential role in the calculation of the drag coefficient $\left(C_{d}\right)$ at the circumstellar medium, we will discuss that in the section3.5.

\subsection{Radiation Parameters}

Radiation has spectral and directional features, that makes its description complex, and scientists have to address both of these features when dealing with radiation problem,see Figure3.3. In addition, Radiation is a light that has dual characters tics (wave and mass),so it a pocket of energy (photons) that has momentum equals to $(h / \lambda)$ and energy $(h \nu)$. Where $h$ is Planck's constant, $\lambda$ is the photon wavelength, and $(\nu)$ is the photon frequency. Since radiation is a spectral and directional property we have to quantify its value by those two terms. Let us start with radiation intensity $(I)$. 


\subsubsection{Radiation Intensity}

radiation intensity(I) at specific point (aperture) in the space is the the energy $\left(\Delta E_{R}\right)$ passing through a unit aperture area $\Delta A_{1}$ and through unit time $(\Delta t)$ per unit solid angle $\left(\Delta \omega=\frac{\Delta A_{2}}{r^{2}}\right)$ For specific band or frequency $(\Delta \nu)$ [3].In symbols, Radiation intensity is:

$$
I_{\nu}=\frac{\frac{\Delta E_{R}}{\Delta t}}{\Delta A_{1} \frac{\Delta A_{2}}{r^{2}} \Delta \nu}
$$

$A_{1}$ should be perpendicular to the horizontal direction of light bundle. The solid angle subtended by $A_{2}$,which is also normal to the bundle direction. Intensity calculations in the radiation field is imperative and it has to be considered through Radiation transport Equation (RTE), this equation has different level of complexities, an assumption is adopted in this research that the Radiation Intensity is constant, or say Radiation flux proportional inversely with distance from the center of the star to computational point in physical domain.It is reasonable to make such assumption due to the fact that density is very small.

The emissive Flux $\left(E_{R}\right)\left[\mathrm{Watt} / \mathrm{m}^{2}\right]$ from a star can be calculated if we know its intensity, by using Equation 3.8.

$$
E_{R \lambda}=\int_{0}^{\infty} \int_{0}^{2 \pi} \int_{0}^{\frac{\pi}{2}} I(\theta, \phi, \lambda) \cos (\theta) \sin (\theta) d \theta d \phi d \lambda
$$

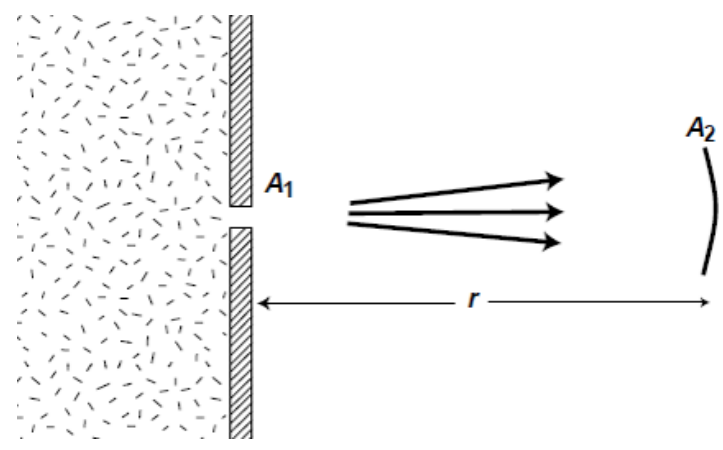

Figure 3.4: Illustration of the ideal apparatus that serves to define the specific intensity[3] 


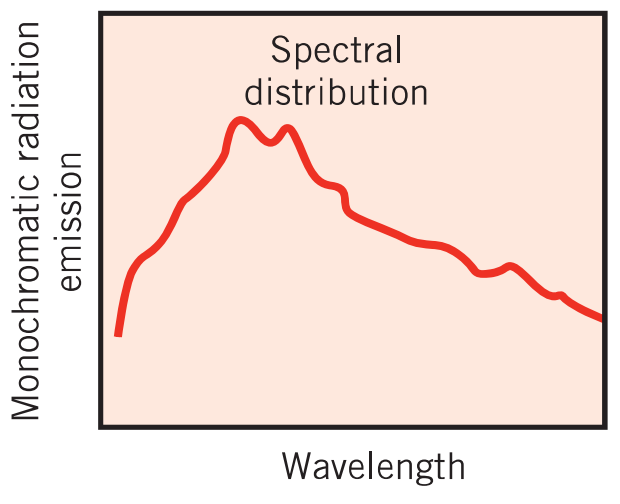

(a)

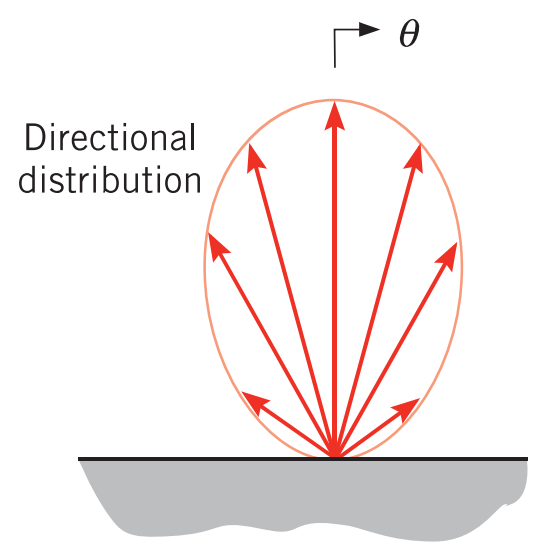

(b)

Figure 3.3: Radiation emitted by a surface. (a) Spectral distribution. (b) Directional distribution[40]

Where $\theta$ is the angle between differential bundle direction and the plane of area $A_{1}, \sin (\theta)$ and $\cos (\theta)$ are seen in the equation 3.8 to make differential bundle seen in Figure 4.7 perpendicular to area $A_{1} \& A_{2}$ respectively.

AGB stars are considered a black body (emits diffusely and follow Planck distribution for spectral intensity). Since black body has constant intensity when the temperature is constant. The total emissive flux is as follows.

$$
\boldsymbol{E}_{\boldsymbol{R}}=\pi \boldsymbol{I}=\sigma T_{s}^{4} \boldsymbol{n}
$$

Where $\sigma$ is Stefan-Boltezman's constant $\left(5.67 \times 10^{-8}\right.$ watt $\left./\left(m^{2} K^{4}\right)\right)$ and $T_{s}$ is AGB star temperature. $\boldsymbol{n}$ is position unit vector. Radiation is not only heating the gas and dust (particles)due to absorption. Gas and particles absorb, reflect, and emits the radiation, it, also, push gas and dust in outer space. In the next section, we will simplify our assumptions towards these complex phenomena. Also, we will address the radiation pressure in simple way such that it will be it easy to grasp. 


\subsubsection{Radiation Pressure}

Radiation is light, and light is a bundle of photons with different energy levels. Each photon carries momentum $\left(\frac{h}{\lambda}\right)$ or $\left(\frac{h . \nu}{c}\right)$ by using $(c=\lambda \nu)$, where $h$ is Planck constant $\left(6.626 \times 10^{-34} j . s\right), c$ is the speed of light $(300000 \mathrm{~km} / \mathrm{s}$ and $\lambda$ is the photon's wavelength. Since pressure is the rate change of momentum per unit area from Newton's second law and energy rate of the bundle of photons per unit area is $\left(E_{R}\right)$, we can write the radiation pressure term as follow.

$$
R_{T}=\frac{E_{R}}{c}
$$

photons are not pushing gases or dust from one side only. Due to complexity of scattering,absorption and emission, Radiation pressure $R_{P}$ would equal Radiation pressure term by Radiation Pressure Crossection (RPCS) $C_{R}$. For black body, $E_{R}=$ $\sigma T_{s}^{4}$, and $R_{P}$ will be then as follows.

$$
R_{P}=C_{R} R_{T}=C_{R} \frac{E_{R}}{c}=C_{R} \frac{\sigma T_{s}^{4}}{c}
$$

The calculation of RPCS is complicated. It depends on various parameters, like particle size, and light wavelength, refractive index, etc [41]. If we have a complete absorption from only one direction, $C_{R}=1$, also, if we have a complete reflection to the radiation from only one direction $C_{R}=2$. In fact, we are dealing with medium (gas and dust) that interacts with radiation by absorption and scattering, and the radiation between gas and dust is quite complicated, and scattering, absorption are existed from all directions[42]. In this research, we did consider simple exponential decay due to scattering and absorption on gas and particles while solving Radiation transport Equation (RTE). However, we took care of these extinction parameters while calculating RPCS because the radiation pressure comes from absorption and scattering of incident radiation on gas or particles[43] [44]. 
Equation 3.11 gives the pressure at the surface of the star, but for different particles location the value of radiation pressure will be different because the received radiation by each particle rely on the their orientation relative to the star. It is essential to find the view factor (see Figure 3.5) from each particle to the star this can be simply done using the following equation[45].

$$
F_{S-P}=\frac{R^{2}}{D^{2}} \cos (\theta)
$$

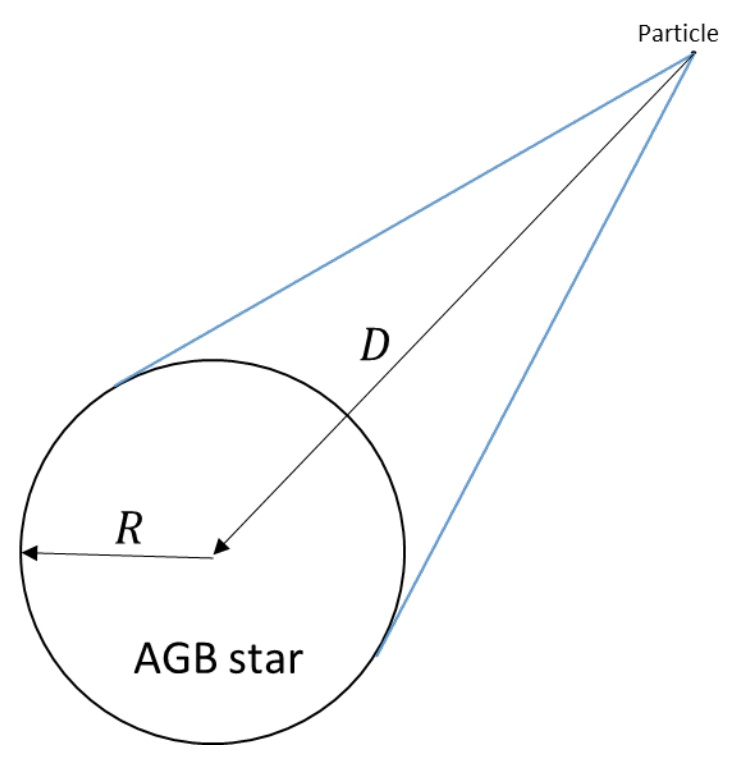

Figure 3.5: View Factor of fireball, particle sees the whole star disk at any $\operatorname{position}(\cos (\theta)=1.0)[45]$

Where $\theta$ is the angle between normal to the observer plane at particle position with direction of radiation. Since the particle has small size $d \sim 10^{-4} \mathrm{~cm}$ it is considered as a point,it doesn't have any specific orientations or say $\theta=0$.

The radiation pressure in Equation 3.8 has to be modified by view factor in Equation 3.12 . 


\subsubsection{Radiation Acceleration}

Once we calculate the radiation pressure on the gas and or the particles, we can easily find the acceleration subjected to the matter as follow.

$$
\boldsymbol{a}_{\boldsymbol{R P}}=\frac{R_{P} A_{p}}{M_{p}} \boldsymbol{n}
$$

Where $A_{p}$, and $M_{P}$ are the cross-sectional area, and the mass of the particle respectively. $\boldsymbol{n}$ unit vector from center of big object to the spatial location of particle, While calculation of gas acceleration is slightly different than the one on the particle[46].

$$
\boldsymbol{a}_{\boldsymbol{R} G}=k_{e x t} \frac{A_{G} E_{R}}{r^{2} c} \boldsymbol{n}
$$

Where $A_{G}$ is grid cell area and $r$ radial distance from cell center to the star center.

\subsection{Gravity}

Gas body force due to gravity $g$ can be calculated using point mass model, In such case $\vec{g}$ will be.

$$
\boldsymbol{g}=-\frac{G M_{s}}{r^{2}} \boldsymbol{n}
$$

Where $G=6.6743015 \times 10^{-11}\left[\mathrm{~m}^{3} \mathrm{~kg}^{-1} \mathrm{~s}^{-2}\right]$ is gravitational constant. $M_{s}$ mass of the star. $r$ is the distance between center of big object and spatial location of gas flow. $\vec{n}$ unit vector from center of big object to the spatial location of gas cell center. The negative sign refers to the inward direction of gravity towards the AGB star. Same model is used for particles. Since particles will be solved in Lagrangian system of equations, the positions of moving particles differ than the fixed cell center location, Normal vector will be different following the particles locations. 


\subsection{Governing Equations}

Since we are dealing with E-L systems of equations, conservative equations for Eulerian gas flow and Lagrangian particles motion are solved separately. However, continuity, momentum, and energy equations for both systems are linked through source terms. These mass, momentum and energy sources carry mapped variables from one system to another. To make two-way coupling of multi-phase particle in cell method (MP-PIC. If we cancel the viscous terms in Naiver-Stocks equations, we will get Euler's equations, which can be written after adding source terms as illustarted in the next sections.

\subsubsection{Eulerian Gas System}

Continuity, momentum and energy equations for gas, multi-species with radiation and particles source terms as follow.

$$
\begin{gathered}
\frac{\partial \epsilon \rho_{g}}{\partial t}+\nabla \cdot \epsilon \rho_{g} \boldsymbol{v}_{\boldsymbol{g}}=0 \\
\frac{\partial \epsilon \rho_{g} \boldsymbol{v}_{\boldsymbol{g}}}{\partial t}+\nabla \cdot \epsilon \rho_{g} \boldsymbol{v}_{\boldsymbol{g}} \boldsymbol{v}_{\boldsymbol{g}}+\nabla P=-\boldsymbol{F}_{\boldsymbol{s}}+\epsilon \rho_{g}\left(\boldsymbol{g}+\boldsymbol{a}_{\boldsymbol{R} \boldsymbol{G}}\right) \\
\frac{\partial \epsilon \rho_{g} E}{\partial t}+\nabla \cdot\left(\epsilon \rho_{g} E+p\right) \boldsymbol{v}_{\boldsymbol{g}}+\frac{P \partial \epsilon}{\partial t}=\epsilon \rho_{g} \boldsymbol{v}_{\boldsymbol{g}} \cdot\left(\boldsymbol{g}+\boldsymbol{a}_{\boldsymbol{R} \boldsymbol{G}}\right)+E_{s} \\
\frac{\partial \epsilon \rho_{g} X_{l}}{\partial t}+\nabla \cdot \epsilon \rho_{g} X_{l} \boldsymbol{v}_{\boldsymbol{g}}=0 \\
\sum_{l} X_{l}=0
\end{gathered}
$$

In equations (3.16)-(3.20), $\epsilon$ and $E$, are the gas void fraction, and total energy of 
gas phase, $p$ and $\boldsymbol{g}$ are pressure and gravity, $\boldsymbol{F}_{\boldsymbol{s}}$ and $E_{s}$ are momentum and energy source terms, $\boldsymbol{a}_{\boldsymbol{R}}$ is radiation acceleration. These sources carry the effects of the particles,radiation to the gas phase. These equations are solved using the directional splitting or Strang splitting method. In this method, the conservative equations are solved in one direction first, then the multidimensional implementation is considered. For more details, see Fryxell et al. 2000 [47].

The above equations can be rewritten again in dimensional splitting form as follow.

$$
\begin{gathered}
\frac{\partial \epsilon \rho_{g}}{\partial t}+\frac{\partial A \epsilon \rho_{g} u_{g}}{\partial V}=0 \\
\frac{\partial \epsilon \rho_{g} u_{g}}{\partial t}+\frac{\partial A \epsilon \rho_{g} u_{g} u_{g}}{\partial V}+\frac{\partial p}{\partial \zeta}=-F_{s}+\epsilon \rho_{g} g \\
\frac{\partial \epsilon \rho_{g} v_{g}}{\partial t}+\frac{\partial A \epsilon \rho_{g} u_{g} v_{g}}{\partial V}=0 \\
\frac{\partial A\left(\epsilon \rho_{g} E+p\right) u_{g}}{\partial V}+\frac{p \partial \epsilon}{\partial t}=\epsilon \rho_{g} u_{g} g+E_{s} \\
\frac{\partial \epsilon \rho_{g} X_{l, g}}{\partial t}+\frac{\partial A \epsilon \rho_{g} X_{l, g}}{\partial V}=0 \quad \text { for } l \neq v \\
\frac{\partial \epsilon \rho_{g} X_{v, g}}{\partial t}+\frac{\partial A \epsilon \rho_{g} X_{v, g}}{\partial V}=0
\end{gathered}
$$

Equations (3.21)-(3.26) are solved two times using Strang splitting first when $\zeta=x$ then when $\zeta=y$, if we are dealing with a three-dimensional problem we have to solve the above equations when $\zeta=z$ as well. $u_{g}$ is velocity components of gas towards $\zeta$ direction and $v_{g}$ is perpendicular to $\zeta$ direction. $X_{l, g}$ mass fractions of index $l$ except vapour and,$X_{v, g}$ is mass fraction of water vapour, $g$ and $a_{R G}$ are not in bold which means those are accelerations in $\zeta$ component (bold symbol means vector quantity). 
The above equations are solved using the control volume approach with the piecewise parabolic method (PPM) as a discretization scheme. The PPM method has four steps.

a) Reconstruction, b) contact discontinuity steepening, c) flattening, and d) solution. The discretized forms of the above equation with details are described in Fryxell et al. 2000 [47]. Before starting the gas flow solution, we should have the source terms calculated first. This will be done by mapping Lagrangian system variables to the Eulerian system. Section 3.4.2 will address Lagrangian system.

\subsubsection{Lagrangian System}

Particle dynamics is solved starting with the Liouville equation for the particle distribution function (PDF) $\phi$ as given in Andrews \& O'Rourke (1996) [18].

$$
\frac{\partial \phi}{\partial t}+\nabla \cdot\left(\phi \boldsymbol{v}_{\boldsymbol{p}}\right)+\nabla_{v_{p}} \cdot\left(\phi \boldsymbol{A}_{\boldsymbol{p}}\right)=0
$$

Where $\boldsymbol{A}_{\boldsymbol{p}}$ and $\boldsymbol{v}_{\boldsymbol{p}}$ are acceleration and velocity of particle respectively.The accelration of particle is given by.

$$
\boldsymbol{A}_{\boldsymbol{p}}=\frac{\boldsymbol{d} \boldsymbol{v}_{\boldsymbol{p}}}{d t}=D_{p}\left(\boldsymbol{v}_{\boldsymbol{g}}-\boldsymbol{v}_{\boldsymbol{p}}\right)-\frac{1}{\rho_{p}} \nabla P+\boldsymbol{g}_{p}+\boldsymbol{a}_{\boldsymbol{R} \boldsymbol{P}}+\frac{1}{\alpha \rho_{p}} \nabla \boldsymbol{\tau}
$$

$\tau$ is the interparticle stress, and $\alpha$ is the particle mass fraction, $1 / \rho_{P} \nabla p$ is the acceleration due to bouncy force. The gravity $\boldsymbol{g}_{\boldsymbol{p}}$, Radiation acceleration of particle $a_{R P}$ and the inter-particle stress $\boldsymbol{\tau}$ are not considered in the shock-tube problem in the present study, but gravity and Radiation acceleration are considered in AGB star problem though. $D_{P}$ is acceleration due to drag experienced by particles, which is given by the following equation.

$$
D_{p}=C_{d} \frac{3}{8} \frac{\rho_{g}}{\rho_{p}} \frac{\left|\boldsymbol{v}_{\boldsymbol{g}}-\boldsymbol{v}_{\boldsymbol{p}}\right|}{r_{p}}
$$


In section 3.5 will go through the calculation of drag coefficient $C_{d}$. The absolute is considered to find the scalar quantity of relative velocity of The volume fraction $(\alpha)$ of particles can be calculated using $\operatorname{PDF} \phi$ as follow.

$$
\alpha=\iint \phi v_{p} d m_{p} d v_{p}=\iint \phi \frac{m}{\rho_{p}} d m_{p} d v_{p}
$$

The sum of the volume fraction of particles $(\alpha)$ and the void fraction of the of gas $(\epsilon)$ is unity.

$$
\alpha+\epsilon=1
$$

The momentum source, Equation 3.22, term $\left(\boldsymbol{F}_{\boldsymbol{s}}\right)$ is found by summing the momentum for each parcel acceleration interpolated to the Eulerian mesh zone,see Equation 3.32. The energy source term is calculated in similar way,see Equation 3.33.Both momentum and energy sources are then mapped from particle location to the grid cell center location.

$$
\begin{aligned}
F_{s} & =\iint \phi m_{p}\left[D_{p}\left(\boldsymbol{v}_{\boldsymbol{g}}-\boldsymbol{v}_{\boldsymbol{p}}\right)-\frac{1}{\rho_{p}} \nabla P\right] d m_{p} d v_{p} \\
E_{s} & =\iint \phi m_{p}\left[D_{p}\left|\boldsymbol{v}_{\boldsymbol{g}}-\boldsymbol{v}_{\boldsymbol{p}}\right|^{2}-C_{p, l} \frac{d T_{p}}{d t}\right] d m_{p} d v_{p}
\end{aligned}
$$

The second term in the energy source term equation $C_{p, l} \frac{d T}{d t}$ represents the heat accumulated or lost from particle to the gas, which can be found from energy balance applied on single particle as follows.

$$
m_{p} C_{p, l} \frac{d T_{p}}{d t}=2 \pi r_{p} K_{g} N u\left(T_{g}-T_{p}\right)+\frac{d m_{p}}{d t} H_{f g}+\dot{Q}_{R}
$$

$C_{p}$ is the heat capacity of the particle, $T_{p}$ is the temperature of the particle, $T_{g}$ is the temperature of the gas, $K_{g}$ is the thermal conductivity of the gas, $H_{f g}$ is the latent heat of evaporation which is negected because we didn't consider phase change 
, $N u$ is the Nusselt number,(see Equation 3.35).For more detail of final discretized forms of heat balance, consult Dahal \& McFarland (2017) [48].

$$
N u=\left[2 \epsilon^{-1.75}+0.6 \frac{R e^{1 / 2}}{\epsilon} \operatorname{Pr}^{1 / 3}\right]
$$

Where $R e$ is reynolds number at particle location, and $\epsilon$ is gas volume fraction. $\dot{Q}_{R}$ is the radiative heat transfer which is only considered in AGB star problem. radiation heat transfer between particle and environment can be described as follow.

$$
Q_{R}=A_{p}\left(\varrho_{p} E_{R}-\varepsilon_{p} \sigma T_{p}^{4}\right)
$$

Where $E_{R}$ is radiation flux at particle location (irradiation subjected on particle), $\varepsilon_{p}$ is particle emissivity, and $\varrho_{p}$ is particle absorptivity. Emissivity and absorptivity of particle are related to each for gray body, one can assume that the particles can be considered as a gray body $\left(\varrho_{p}=\varepsilon_{p}\right)$, gray body is can also be opaque surface which means the transmissivity equal to zero. To find $E_{R}$ at particle location, we have to solve the following radiative heat transfer.

$$
\frac{\partial \boldsymbol{I}_{\boldsymbol{\lambda}}}{\partial r}=\boldsymbol{\eta}_{\boldsymbol{\lambda}}-k_{\lambda}^{e x t} \boldsymbol{I}_{\boldsymbol{\lambda}}
$$

Where $\boldsymbol{I}_{\boldsymbol{\lambda}}$ is spectral intensity, $r$ radial distance from center of AGB star to the particle location. $\boldsymbol{\eta}_{\boldsymbol{\lambda}}$ is the value of intensity increase by scattering and emission from other sources(different than AGB star), and $-k_{\lambda}^{e x t}$ is intensity extinction due to absorption and scattering. These values are also directional and spectral properties. Constant average values are assumed for these parameters. These assumption are considered for simplicity and we might be going out of the topic scope if we address these parameters in details. If we consider zero effects of other outer space radiation $\boldsymbol{\eta}_{\boldsymbol{\lambda}}=0$ and average constant value of $-k_{\lambda}^{e x t}$. 


\subsection{Drag Models}

In the present study, we used two different drag models: continuum model, used by O'Rourke and implemented by Dahal \& McFarland (2017) in FLASH[18][21][23]. Non-continuum model, derived using kinetic theory in Nearly Free Molecular Flow and implemented in Flash in the current study [49].

\subsubsection{Continuum Model}

The large-scale formation of the SDMI depends on particle-scale transport of momentum, energy, and mass. Of these, momentum transfer has the largest effect. Energy transfer has a smaller effect. For momentum transport, the simple drag model considers a spherical rigid particle with size, $r_{p}$, mass, $m_{p}$, and velocity, $\mathbf{v}_{\mathbf{p}}$ in a gas with viscosity, $\mu_{g}$, and velocity, $\mathbf{v}_{\mathbf{g}}$. The particle Reynolds number is calculated as follow:

$$
R e=\frac{2 \rho_{g}\left|\boldsymbol{v}_{\boldsymbol{g}}-\boldsymbol{v}_{\boldsymbol{p}}\right| r_{p}}{\mu_{g}}
$$

where $v_{p g}$ is the magnitude of the particle velocity relative to the gas. The force on a particle is then.

$$
\boldsymbol{F}_{\boldsymbol{d}}=m_{p} D_{p}\left(\mathbf{v}_{\mathbf{g}}-\mathbf{v}_{\mathbf{p}}\right)
$$

Where $\left|\boldsymbol{v}_{\boldsymbol{g}}-\boldsymbol{v}_{\boldsymbol{p}}\right|$ is a scalar component of the acceleration due to drag, $D_{p}$ is defined in equation 3.29. $C_{d}$ is the drag coefficient for the particle and is calculated using various method dependent on $R e$, particle volume fraction, and Mach number.

$$
\begin{aligned}
& C_{d}=\frac{24}{R e}\left(\epsilon^{-2.65}+\frac{R e^{\frac{2}{3}}}{6} \epsilon^{-1.78}\right), R e<1000 \\
& C_{d}=0.424, \quad R e>1000, \quad M a<0.3 \\
& C_{d}=0.424+\frac{1-0.47}{1.5-0.3}\left(\begin{array}{c}
M a-0.3), \quad 0.3<M a<1.5 \\
40
\end{array}\right.
\end{aligned}
$$




$$
C_{d}=0.884, \quad M a>6.0
$$

Continuum model is used in the shock-tube problem. In general drag coefficient in this model relies on Reynolds number rather than Mach number.

\subsubsection{Non-Continuum Model}

Reynolds number has a significant role on the drag model in continuum model. However, for non-continum (rarefaction), we can asses the flow type by magnitude of the Knudson Number $K_{n}$. Drag in rarefaction condition rely on Knudson Number, which is in the end a function to both $R e$ and $M a$ numbers, see equation 3.6. In the free molecular regimes, where $K_{n}>10$. The drag is calculated by considering collision of molecules to the surface of particles. Boltzmann equation must be directly treated by appropriate numerical techniques such as the Direct Simulation Monte Carlo (DSMC) or the lattice Boltzmann methods (LBM). In this model, Numerical solution of Boltzmann equation was used to find the drag coefficients on sphere at which $C_{D}$ will have an expansion as follow :-

$$
C_{D}=C_{o}+C_{1} K n^{-1}+C_{2}^{\prime} K n^{-2} \log \left(K n^{-1}\right)+C_{2} " K n^{-2}+\ldots
$$

Where $C_{o}, C_{1}, C_{2}$ are coefficients of expansion which depend on speed ratio $S$, which is the ratio of relative velocity $\left(\left|\boldsymbol{v}_{\boldsymbol{g}}-\boldsymbol{v}_{\boldsymbol{p}}\right|\right)$ to the thermal molecular velocity $\left(\sqrt{\frac{2 k_{B} T}{m_{m}}}\right)$, where $m_{m}$ is molecular mass, and $k_{B}$ is Boltzmann's constant. Speed ratio can be rewritten in terms of Mach number as follow[49].

$$
S=M a \sqrt{\frac{\gamma}{2}}
$$

where $\gamma$ is specific heat ratio of the gas. One special case for the solution of drag $C_{D}$ in equation 3.46 when the object has small size like dust particle $(\sim 1$ micron $)$ 


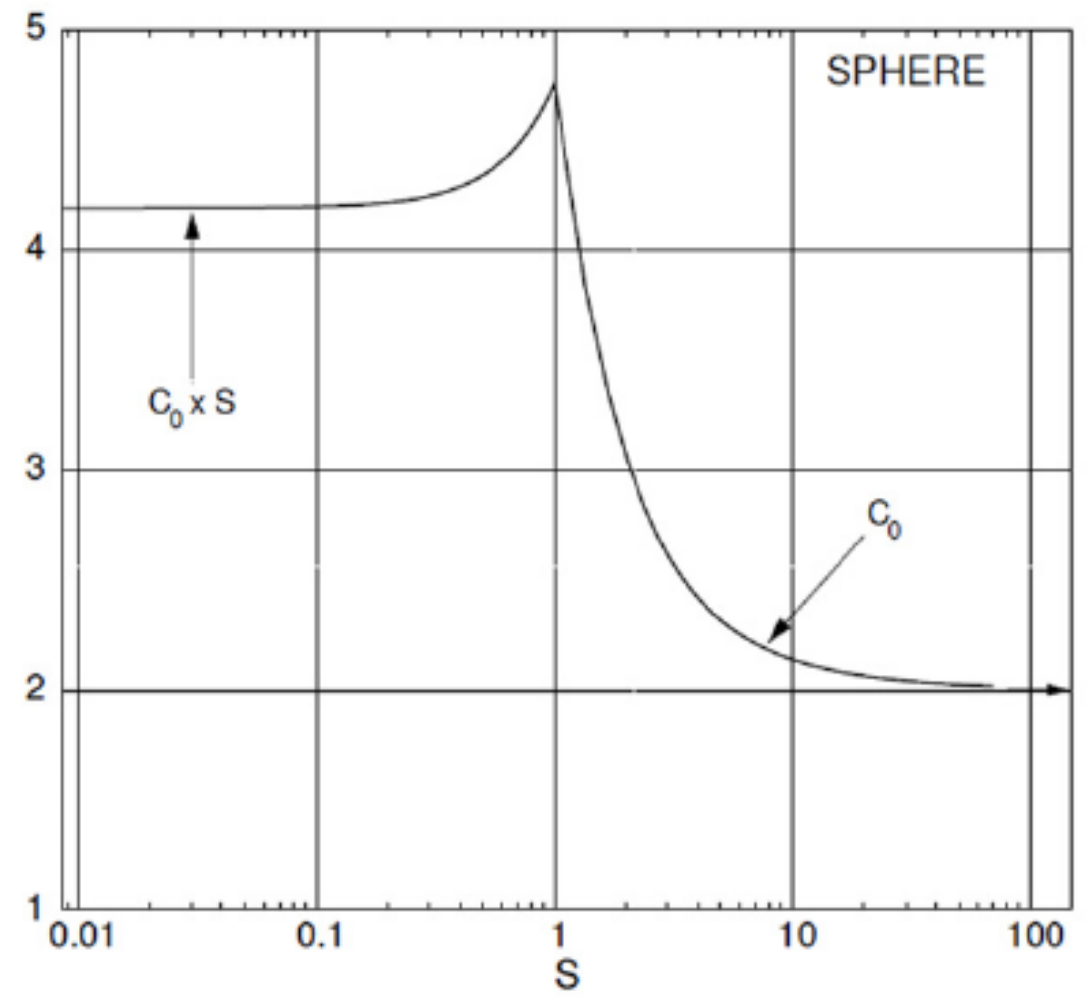

Figure 3.6: Drag coefficients ratio $C_{o}$ of a sphere in free molecular flow as a function of the speed ratio $S[49]$

with large mean free path of gas molecules at outer space (some times $\lambda>1.0 \mathrm{~km}$ ). This will make $K n^{2} \rightarrow \infty$ or $K n^{-2} \rightarrow 0$. Equation 3.46 after neglecting higher terms becomes as follows.

$$
C_{D}=C_{o}+C_{1} K n^{-1}
$$

All what we need is to calculate $C_{o}$ and $C_{1}$. $C_{o}$ is calculated analytically from Boltezmann's distribution of kinetic energy and the result as follows.

$$
C_{o}(S)=\frac{2 S^{2}+1}{\sqrt{\pi} S^{3}} e^{-S^{2}}+\frac{4 S^{4}+4 S^{2}-1}{2 S^{4}} \operatorname{erf}(S)+\frac{2 \sqrt{\pi}}{3 S}
$$

While the other coefficient is numerically estimated. Both coefficients are plotted on log scale to cover large domain of speed ratio (or say Mach Number),see Figure 3.6\& 3.7. This model shows more dependency on mach number than the Reynolds number 


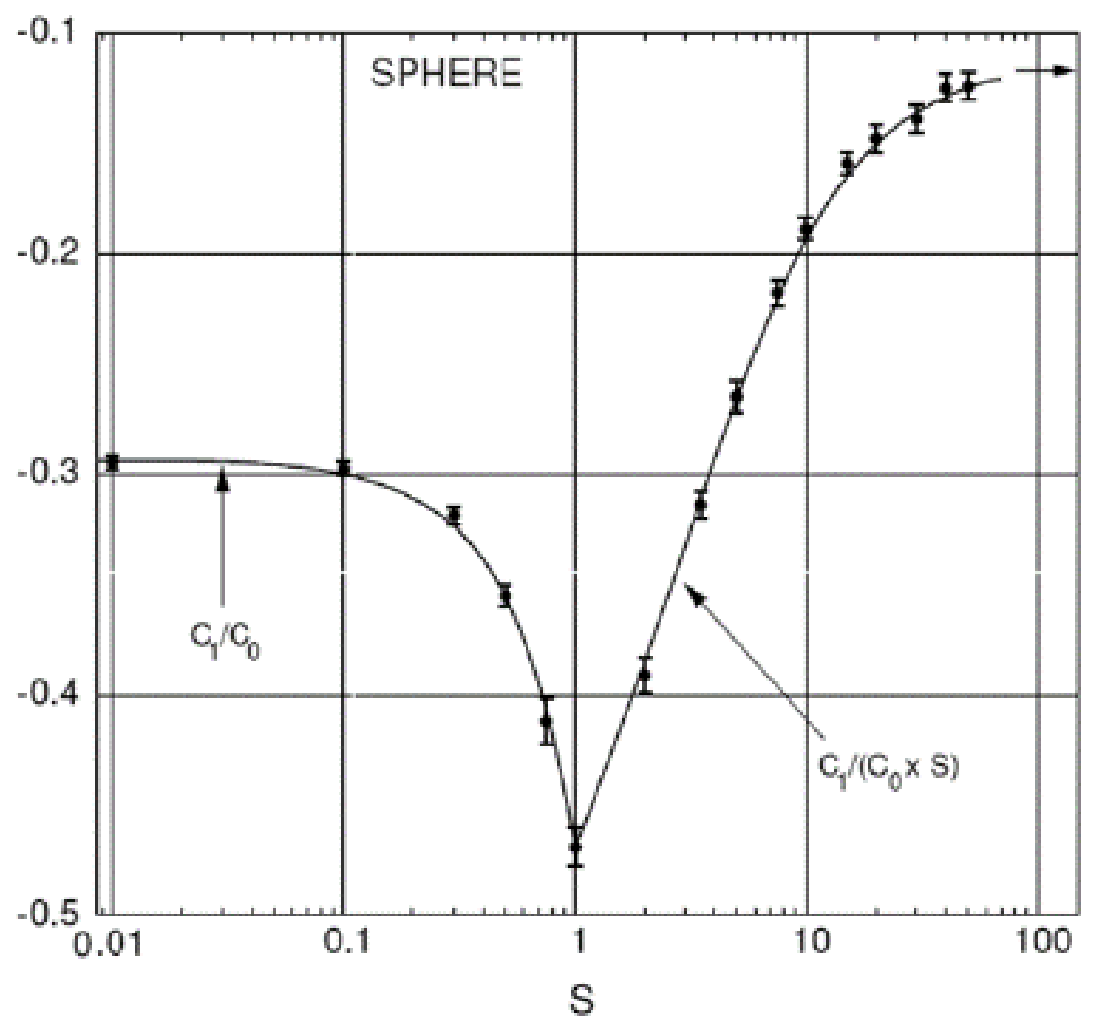

Figure 3.7: Drag coefficients ratio $C_{1} / C_{o}$ of a sphere in free molecular flow as a function of the speed ratio $S$ with error bars[49]

due to rarefaction effect. To apply these equations, gas properties are calculated from flow field first then the dimensionless parameters like Reynolds, Mach, speed ratio, and Knudson number, see Appendix A.3, for more details. 


\section{Chapter 4}

\section{Numerical and Experimental Configurations}

\subsection{Introduction}

In this chapter we will go through preliminary configurations needed for results In chapter 5. We will address the configurations of both problems, shock-tube \& AGB star problem. section4.2 address the geometry of solved problems

\subsection{Building The Geometry}

\subsubsection{Shocktube problem}

We mentioned earlier that our computational domain will be considered only the test section with following dimension $(200 \mathrm{~cm} \times 3 \mathrm{~cm})$, because we are interested in interface evolution occurring in the test section only. See actual size compared with computational size, Figure 4.1A. The actual size seen in Figure 4.1A is $8.3 \mathrm{~m}$ by 0.14 $\mathrm{m}$ and the test section is shown in Figure 4.1B. 


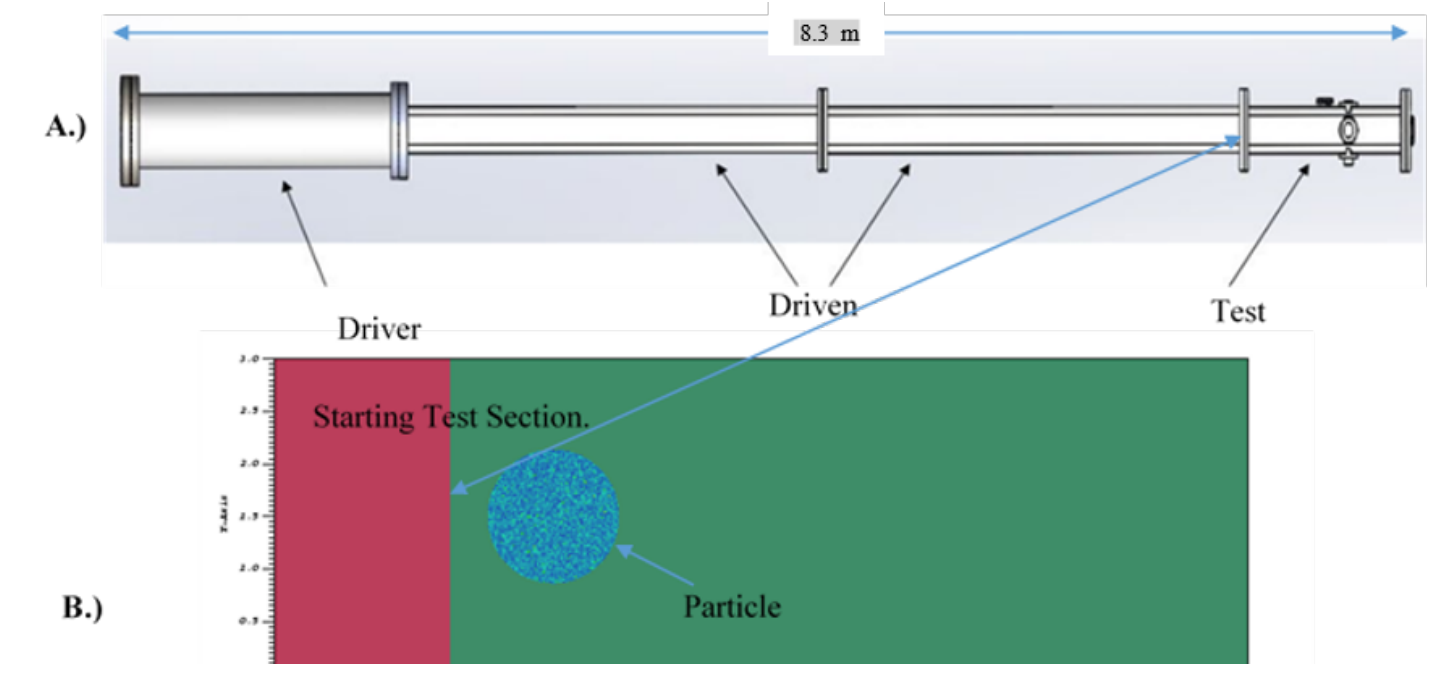

Figure 4.1: A) Actual size of shock tube B) Part of computational size due to high aspect ratio 200:3.

\subsubsection{AGB Star Problem}

The computational domain for AGB stars and CSMs have huge space, as we saw in Figure 1.7. Since we are interested in SDMI phenomena near AGB star, we choose our domain to be within CSM with dimensions $\left(7.5 \times 10^{14}\right.$ by $\left.7.5 \times 10^{14} \mathrm{~cm}\right)$, see Figure 4.2. Our goal in this part of the research is to make good starting steps by making good choices related to geometry and physics. The first choice was to consider a two-dimensional problem before jumping into a three-dimensional setup. We might consider the one-fourth of the domain instead of having the whole domain. However, most of AGB stars have Asymmetric properties[50][10], also, In SDMI terminology that the drag on particles is controlling the morphologies of particles (dust) around the star. Since the particles generated randomly around AGB star, the dust will have different radial interaction causing asymmetric behaviour . 


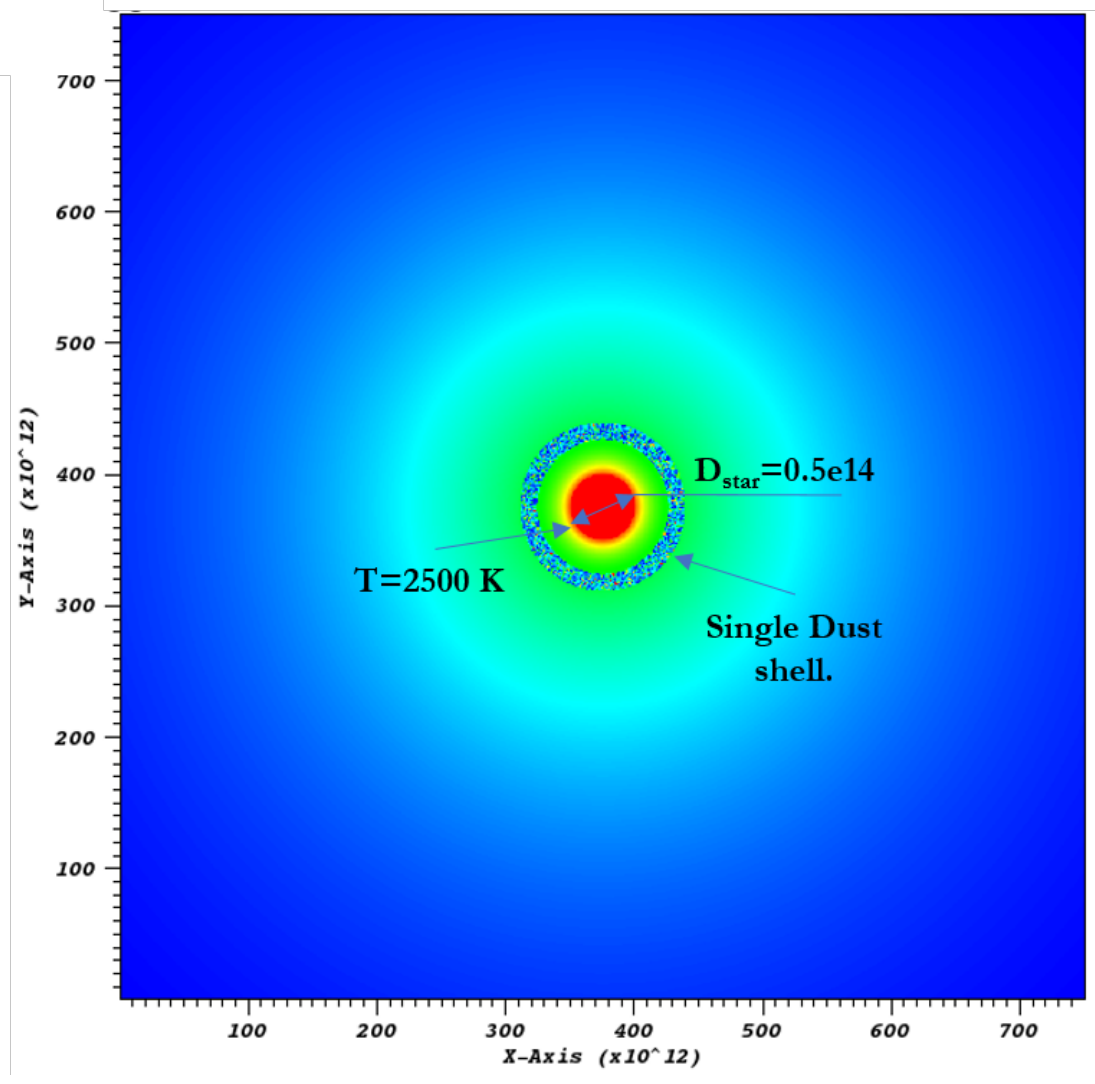

Figure 4.2: Simulation Domain within CSM.

\subsection{Grid Generation}

Flash code is a modular, adaptive, parallel, and multi-physics simulation code handling compressible fluid flow. Parallelization in this code was done by dividing the computational domain to the blocks instead of cells (regular mesh grid), and each block is self-contained, and it is manipulated separately through solutions steps.

Each block will have at least eight cells and surrounded by four cells called guard cells in each direction. The number of guard cells is mandatory for PPM. FLASH uses PARAMESH; this code has dynamic mesh capability and uses different levels of refinement. In the shock-tube problem, we use five levels of refinement, $200 \times 3$ blocks, and each block has $(8 \times 8)$ cells. See Figure 4.3. In the AGB star problem we 
consider $120 \times 120$ blocks with three levels of refinements, see Figure 4.4 .

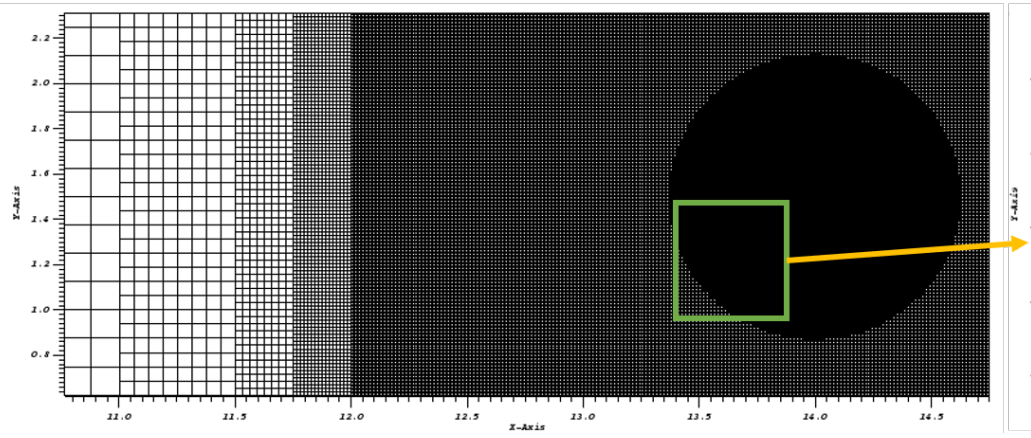

B)

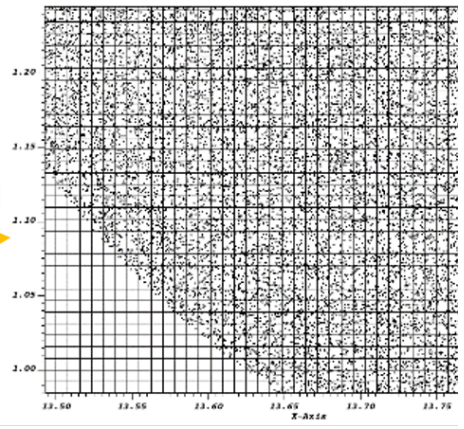

Figure 4.3: A) Shows five levels of refinement. B) Particle grids inside flow grid (PIC).

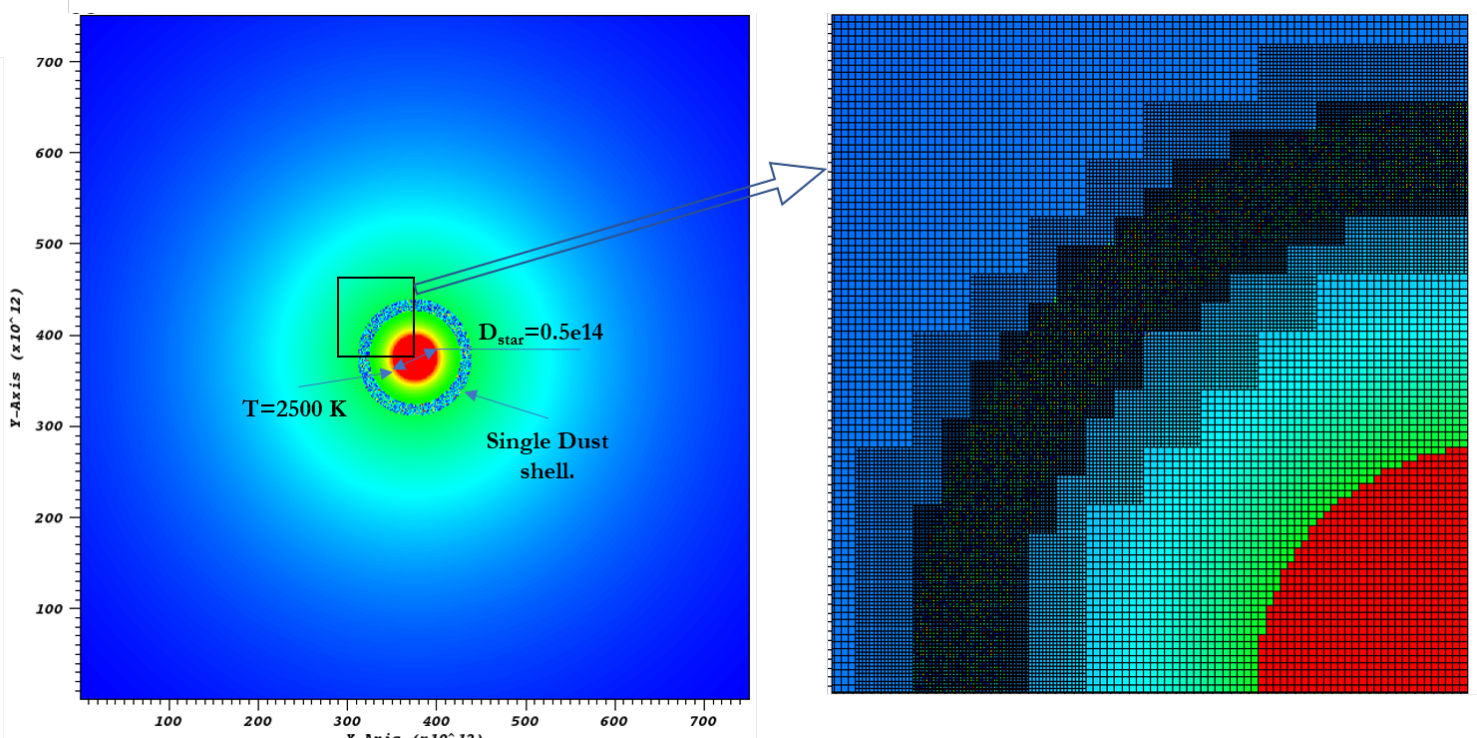

Figure 4.4: Levels of refinement with Particle grids near convection envelope of AGB star.

\subsection{Initial and Boundary Conditions.}

The initial and boundary conditions are set on the test section, as we mentioned earlier. It is useless to spend computational time and memory on regions we are not interested in. We are interested in small scale interface $(1.25 \mathrm{~cm}$ diameter $)$ evolution inside large domain of the shock-tube (driyer, driven sections). As a result, we should 
make our conditions on the test section only, and These need to be close enough to the actual condition . For this reason, we used a one-dimensional theory for the driver and driven section to find the pressure and temperature, density, and Mach number subjected to the test section, see Appendix A.1. A numerical code is implemented for this issue by following shock tube calculations in (Anderson 1990) [37]. the calculated pressure, temperature, and Mach number at pre- and post-shock initial conditions are given in Figure 4.5.

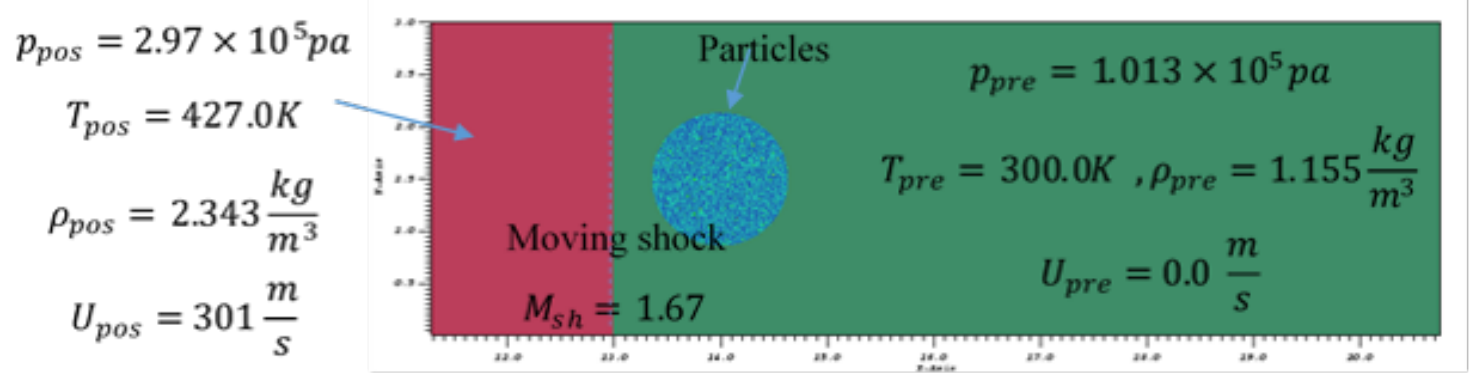

Figure 4.5: Initial conditions of gas flow on test section within shock-tube.

$T_{p o s}, \rho_{\text {pos }}$ and $U_{\text {pos }}$ are post shock conditions, while $T_{\text {pre }}, \rho_{\text {pre }}$ and $U_{\text {pre }}$ are pre-shock conditions.

The above conditions for shock-tube problem. For the AGB star problem we generate our conditions relying on one-dimensional numerical solution models for AGB star without pulsation[51].This solution is good starting point to our simulation because shock waves was not included in their solution. Figutre 4.6 shows the radial distribution of the temperature and density solutions done by Fleischer et al.(1995) and Höfner et al. 


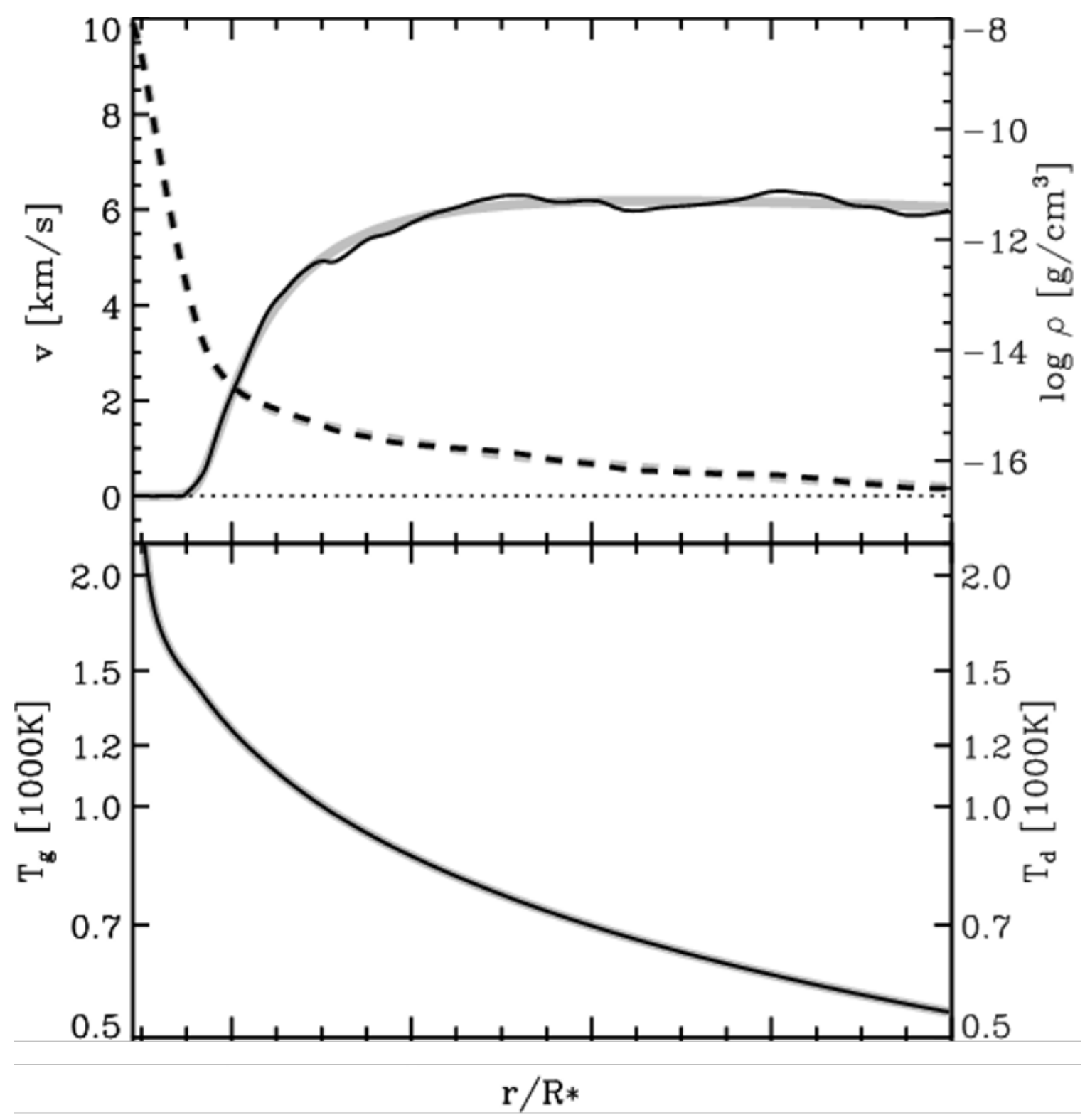

Figure 4.6: The mass density $\rho$ (dashed Top right), the dust and gas temperatures $\left(T_{d}=T_{g}\right.$ in this model) bottom, [46].

Last not least, the radiation field has exponential decay starting from star Temperature $T_{s}=2500$ to the ISM temperature. The radiation field in this study is considered constant, also, it is perturbed by sine wave, see Figure 4.7. 


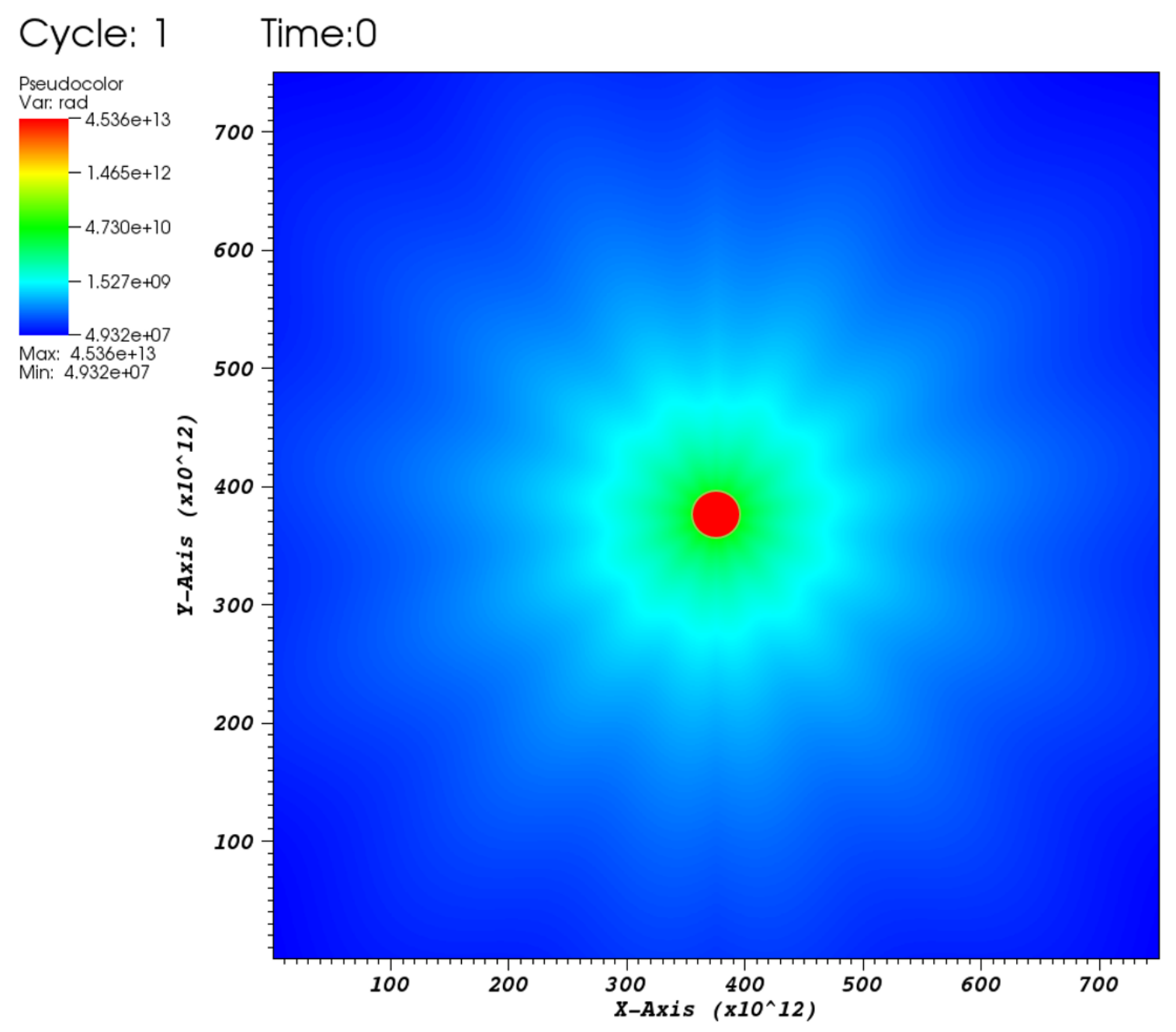

Figure 4.7: Radiation flux field $\left(w a t t / m^{2}\right)$ is radially perturbed.

The above conditions are for the gas flow, but we still need to have our initial conditions in the particles cloud system. Particles generation in this problem rely on the effective Atwood number $\left(A_{e}\right)$ calculations. The Atwood number is measured experimentally first. Then, we use a similar strategy to find number of particles needed to fill our particles region in computational domain with computational points. 


\subsection{Particles Generation.}

To generate particles in computational domain we must consider specific distribution. Scientists have been assumed Scientists have been assumed different distributions for liquid particles. For example, lognormal distribution and Rosin-Rammler (Weibull) and Log-hyperbolic distributions are frequently used distributions in the liquid droplets studies [16]. In this research, we use Log-normal distribution. Lognormal is widely used and easy to deal with, it is derived from standard normal distribution. It is descried by equation4.1.

$$
f\left(r_{P}\right) d r_{p}=\frac{1}{\sqrt{2 \pi} \sigma_{0}} \exp \left[-\frac{1}{2}\left(\frac{l n r_{p}-\mu_{0}}{\sigma_{0}}\right)^{2}\right] \frac{d r_{p}}{r_{p}}
$$

Where $\sigma_{0}$, and $\mu_{0}$ are distribution parameters or say shape parameters. Particles generation in the computational domain are related to the Atwood number calculations and lab conditions (Temperature and Pressure). Pressure and Temperature of the dispersed phase are considered similar to the lab environment condition, assuming particle jet is in thermal equilibrium with lab environment conditions. Calculation of Atwood number are done experimentally first; then we used the calculated one as input to FLASH software. Particle generation is performed and initialized by implementing a FORTRAN code that generates particles and calculates Atwood number sequentially until Atwood number reaches the designated value (from the experimental calculation), See Appendix A.2 for more details. Figure 4.8A shows a log-normal size distribution of the generated particles. see Figure 4.8B, C, and D that shows local uniform distribution of the generated particles. 


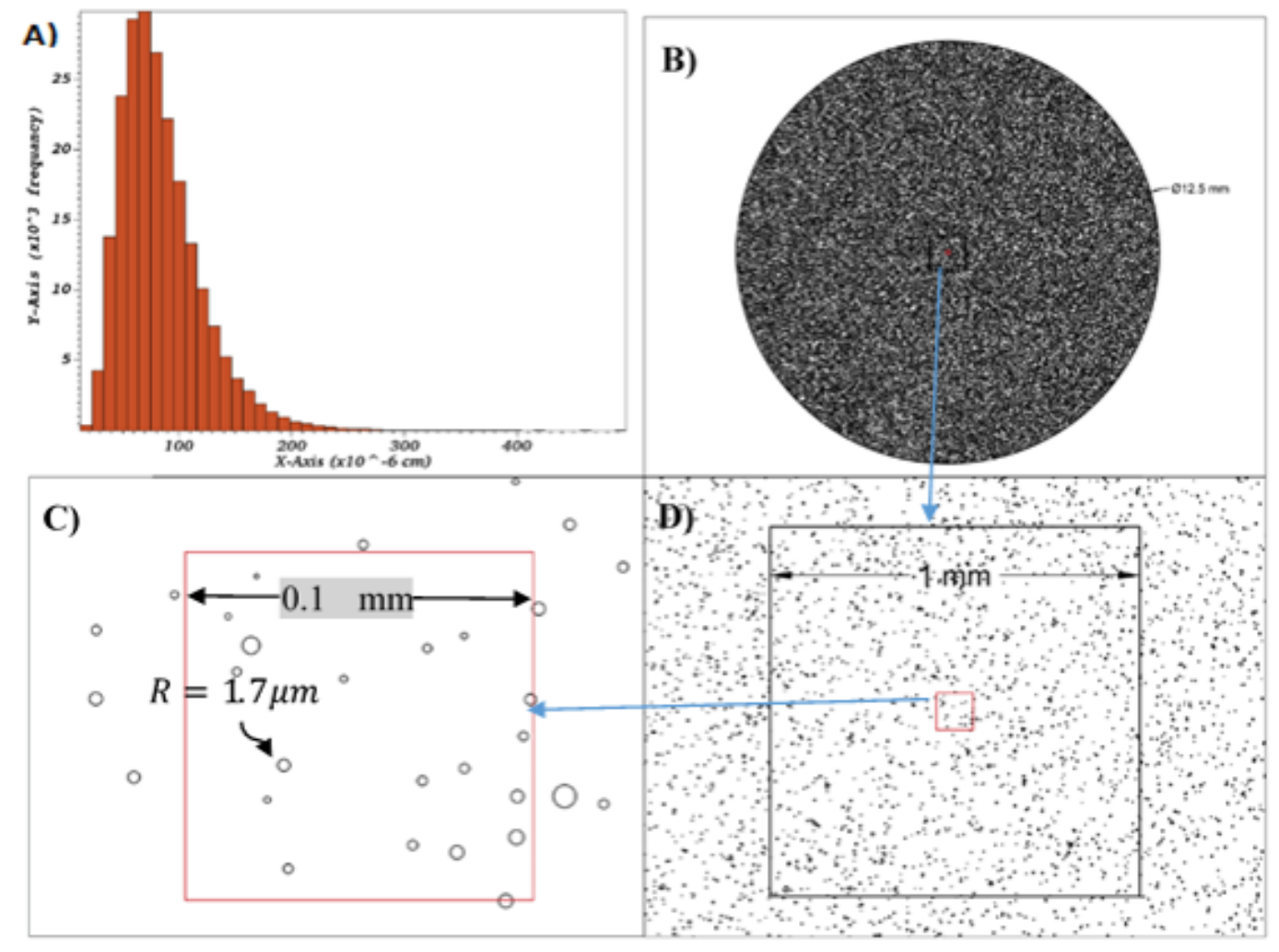

Figure 4.8: A) Size distribution of particles (radius). B) Interface full size. C) Particle size in micro-meter. D) Zoomed in region shows uniform location distribution of particles.

Dust is generated in AGB star problem in single time shell generation. This dust (particles) is uniformly radially distributed and log-normally distributed by size.

The dust distribution is shown in Figure 4.9A. Many graphite particles have a roundish shape and are grown in an onion-like fashion from graphite like sheets particles, see Figure 4.9C. In current study we considered graphite as a type of particles with spherical shape. Dust scatters all but the relatively nearby regions in visual and ultraviolet wavelengths, and re-radiates the absorbed energy in the far-infrared part of the spectrum, thereby providing a major part $(\sim 30 \%)$ of the total luminosity of the Galaxy[52]. The theory of scattering of electromagnetic radiation by small particles that shows particles to strongly scatter radiation only if their size is comparable with or larger than the wavelength of radiation[14], see Figure 4.9B. 

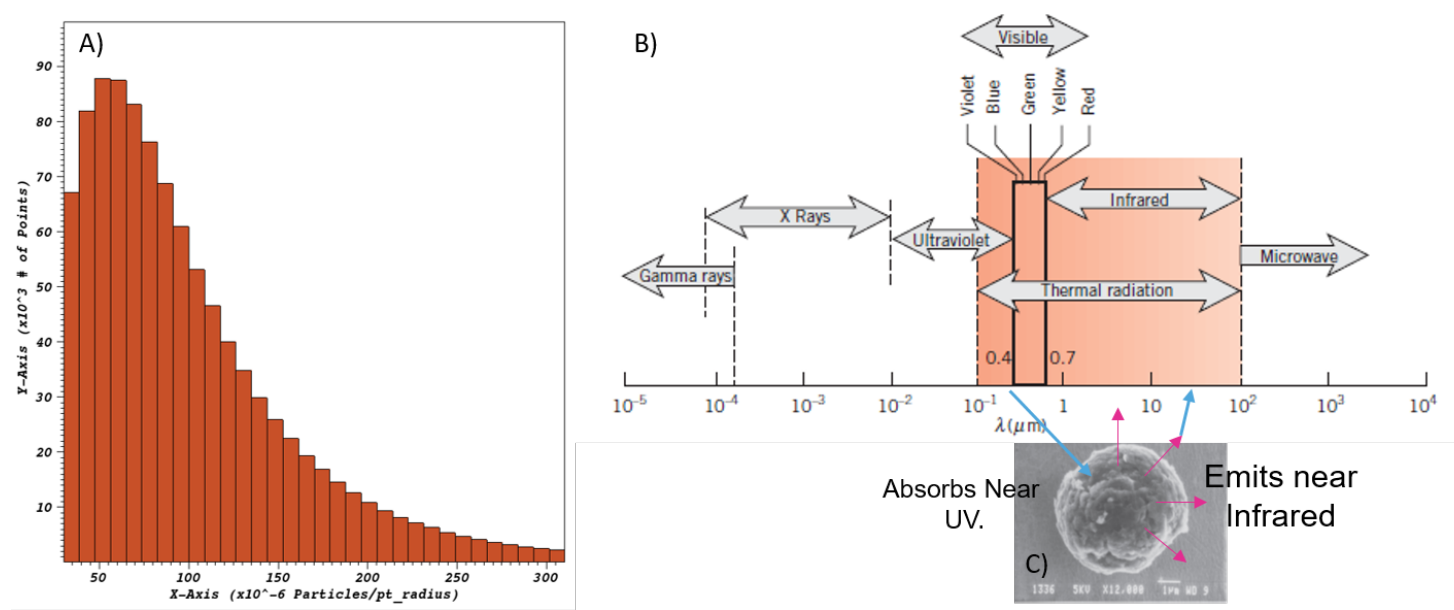

Figure 4.9: A) AGB star Size distribution of particles (radius). B) Radiation spectra shows dust interaction bands[40]. C) Presolar graphite particle isolated from the fine grained matrix material of a meteorite[14].

\subsection{Experimental Calculation of effective Atwood Number.}

This section is explaining calculation of Atwood number that will be considered in experimental setup measurement. Calculation of Atwood number should be done carefully because simple change in the Atwood number will increase or decrease the number of particles needed in the FLASH software inputs. Getting precise Atwood number will have a great effect on SDMI shape and its evolution. For instance, changing Atwood number from $A_{e}=0.01-0.02$. will increase the number of particles by $\% 44.27$ or say increase effective density of second phase and then faster evolution of interface evolves, which means different morphologies are expected during interface histories. 


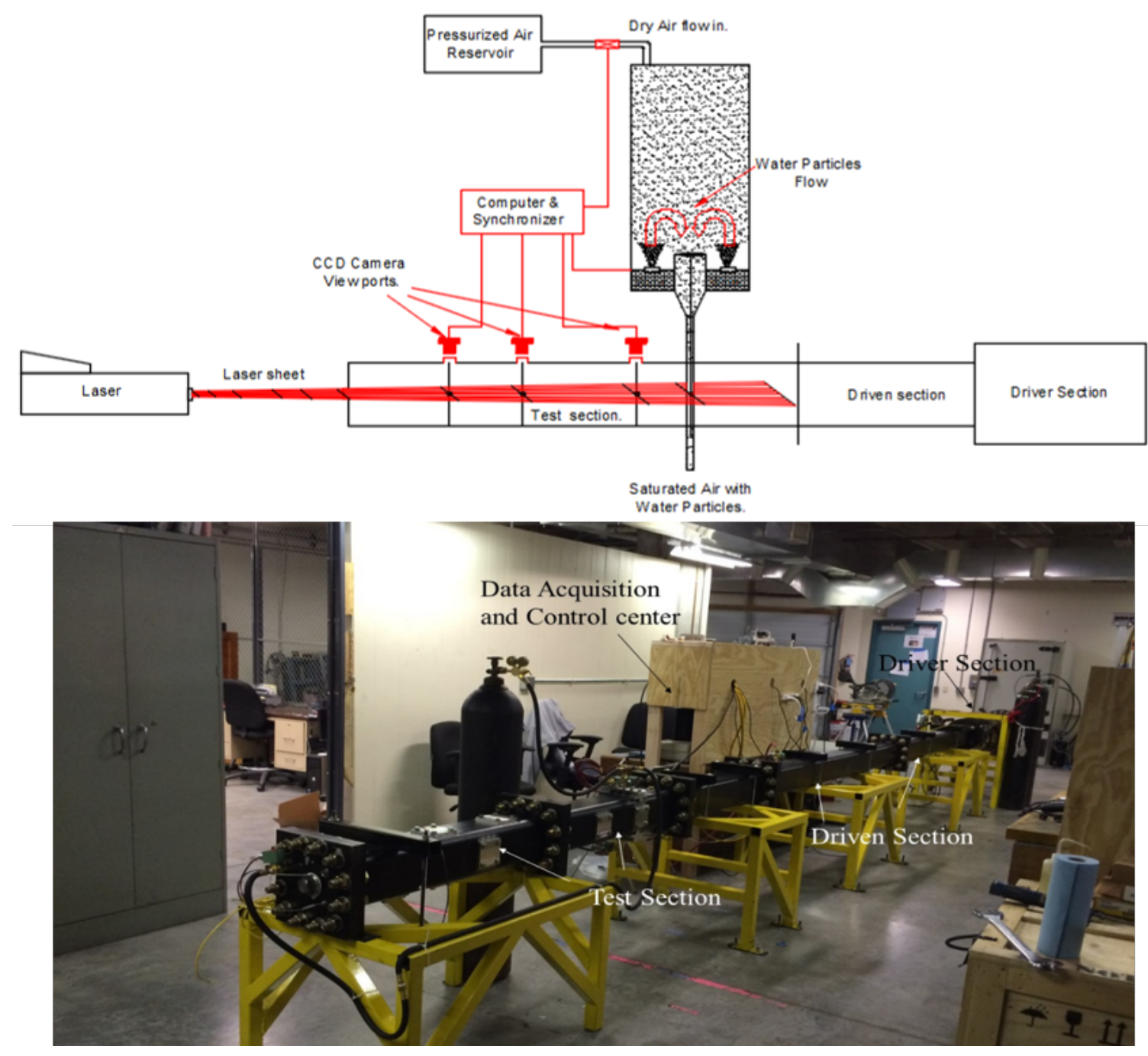

Figure 4.10: A) Schematic of the shock tube facility in university of Missouri, Mechanical and aerospace engineering. B) Actual picture of Shock-Tube.

Figure 4.10 shows schematic of the shock tube facility in university of Missouri, Mechanical and aerospace engineering which consist of (shock-tube, seeding system, capturing system). Each part of this system is set well to get good picture at different test sections ports. The effective Atwood number $A_{e}$ is controlled by dry air flow and the number of atomizers that are running. To do so, we may change either dry air flow rate or number of active working Atomizers. To calculate the Atwood Number, we have to know pre-shock condition of gas inside the shock-tube, and it will be considered as first gas flow condition. These conditions are calculated by assuming the 
following These conditions are calculated by assuming the following These conditions are calculated by assuming the following gases $N_{2}$ and Air. Air is considered with \%15 relative humidity. Using the psychometric chart, we can find the properties of the pre-shock conditions, which are the same as what is given in Figure 4.5. The interface (multi-phase flow) calculation is different from the first one because we have saturated air with water vapor. Water particles are generated in the seeder. If we use thermodynamics relations (mixtures law, mass balance, equation of state), we will find the effective density of the second phase. An implemented code for these calculations is included; see Appendix A.2. 


\section{Chapter 5}

\section{Results}

\section{$5.1 \quad$ Introduction}

In this chapter, we will be going through numerical solutions results of shock tube compared with experimental results done in our Lab by John Middlebrooks et. al first [33]. Then, we will make a quantitative comparisons between simulation results of single shell dust implemented in the current research with AGB star envelops observations by Cox et. al[50].

\subsection{Simulation of the shocktube problem.}

Numerical configurations in previous chapter are essential to initialize the proper conditions for our simulations in both problems. For shocktube problem, table 5.1 summarizes $p, T$, and $\rho$ for pre- and post-shock conditions on the shocktube test section. Mach equals $M_{a}=1.67$, and Atwood number is calculated for two different concentrations $\left(A_{e 1}=0.048 \& A_{e 2}=0.0184\right)$. let us call the large effective Atwood number of the first case, and the small effective Atwood number is the second case. 


\begin{tabular}{|ccccc|}
\hline Initial condition & $T$ & $p$ & $\rho$ & $v_{g}$ \\
\hline Postshock & $427.0 \mathrm{~K}$ & $2.97 \times 10^{5} \mathrm{pa}$ & $2.343 \frac{\mathrm{kg}}{\mathrm{m}^{3}}$ & $301 \frac{\mathrm{m}}{\mathrm{s}}$ \\
Preshock & $300 \mathrm{~K}$ & $1.013 \times 10^{5} \mathrm{pa}$ & $1.155 \frac{\mathrm{kg}}{\mathrm{m}^{3}}$ & $0.0 \frac{\mathrm{m}}{\mathrm{s}}$ \\
\hline
\end{tabular}

Table 5.1: Initial gas condition in shock-tube problem

Before we make comparisons between experimental and numerical results, let us see the morphology development of the another case say $\left(A_{e}=0.0123\right)$. This case is to illustrate most physics that we know about SDMI development.

Figure 5.1 shows the histories of the SDMI's at different time steps. These results are expected from the background information of SDMI; it also shows baroclinic-like effects being maximized at the top and the bottom of the perturbed interface. Figure 5.1A shows high pressure or density region in red color versus atmospheric condition in blue color. It also shows particle local and size distributions. The circular shape of the interface is pushed by gas flow with speed $v_{g}=301$ and squeezed, making elliptical geometry after passing moving shock. Figure 5.1B shows how the big particles lag behind small particles, and the small particles can be considered as flow tracer and big particles, however, are making a crescent-like shape withstanding post shock gas flow.

At $0.5[\mathrm{~ms}]$ interface is developing two vortices at the elliptic-like shape vertices due to high baroclinic-like torque effects. Sizes of deposited vortices increases with time making different mythologies due to the SDMI effects. Once the interface is hit by moving shock wave, it will be then subjected to the expansion waves. These waves, however, are important to give pressure gradients required for holding SDMI ingredients which empower vortex motion. In the end, shock effects will not last long time due to extinction of incident shock effects because of the wall boundary effects at the end of the shock-tube, see Figure 5.1E \&F. In Figure 5.1G\&H there is considerable changes in the interface development because of the reflected shock wave travelling in the opposite direction, see red color in same figure. Reflected shock is 


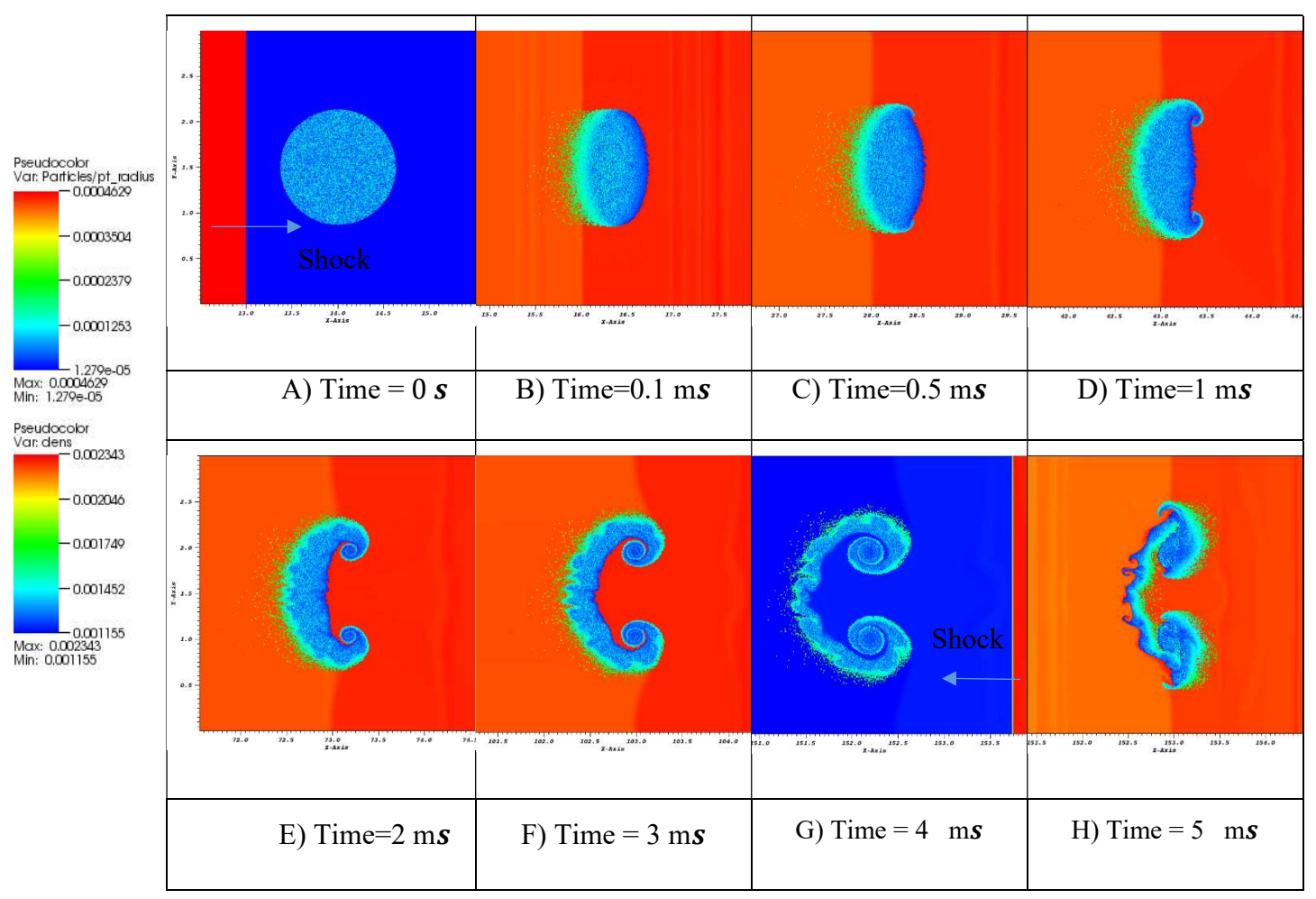

Figure 5.1: Different time steps of interface evolution subjected to incident shock wave with Mach $M_{a}=1.67, A_{e}=0.0123$.

initiating many small scales instabilities and increasing the mixedness of the flow and turbulent flow might be involved which is out of the current research. we will leave re-shock instabilities development for future research.

\subsubsection{Comparisons with the experimental Results.}

The dispersed phase (water particle \& gas flow) flows out of the seeder crossing the shock-tube vertically with cylindrical geometry diameter cross-sectional area. The experimental setup allows changes in the interface shape only in the plane, which is parallel to the laser sheet plane shown in Figure 4.10A. Laser illuminates seeded particles at the middle of shock-tube height (half of the cylindrical column) with specific thickness letting $9 \mathrm{MP}$ CCD camera easy to catch interface development at different 

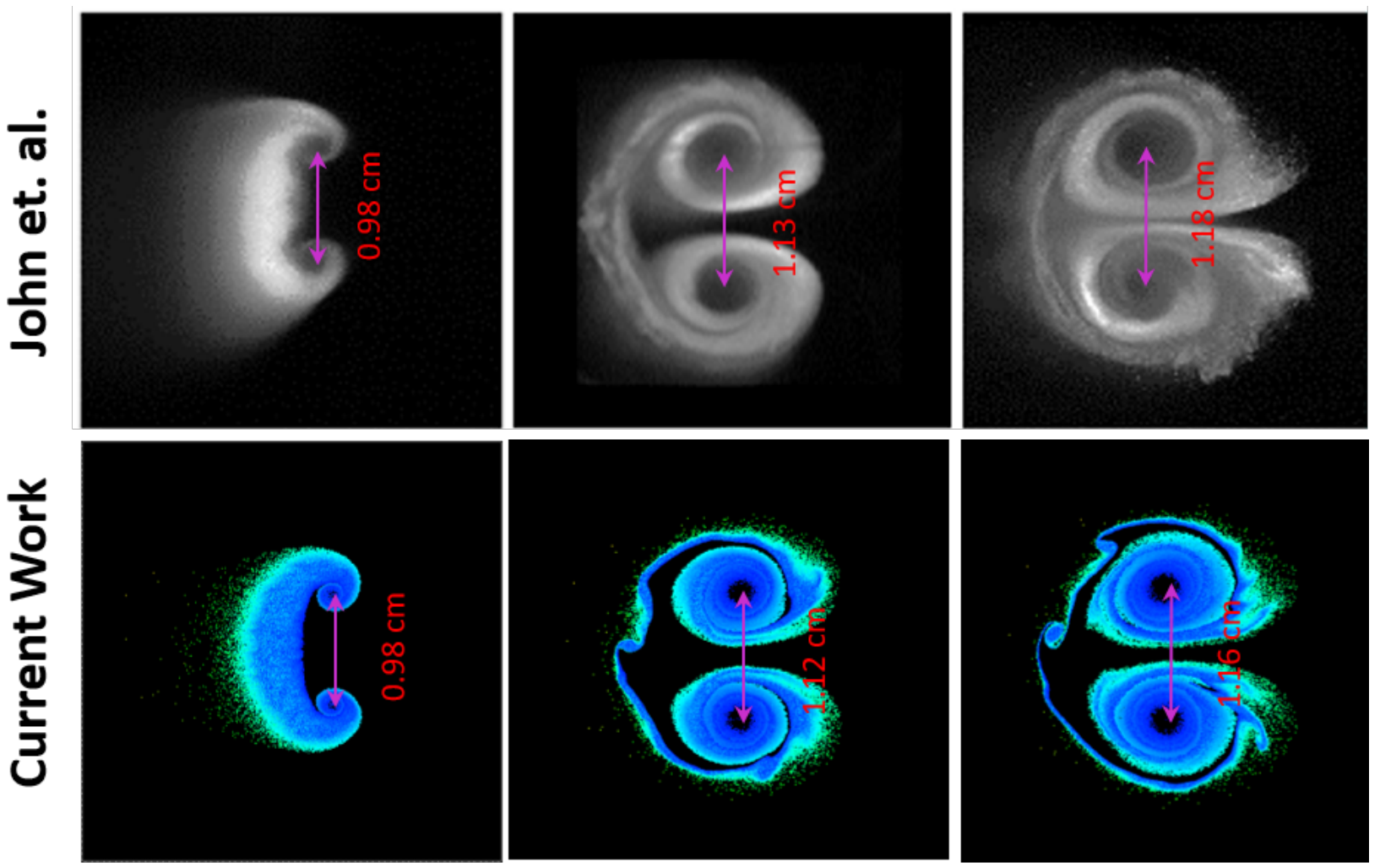

Time $=0.5 \mathrm{~ms}$

Time $=\mathbf{2} .9 \mathrm{~ms}$

\section{Time $=4 \mathrm{~ms}$}

Figure 5.2: Comparisons of SDMI mosaic between experimental results, first Row and numerical solution second row for particle distribution parameters with , $\mu_{0}=1.7 \mu \mathrm{m}$ and $\sigma_{0}=0.728 \mu \mathrm{m}$ and Atwood number $A_{e 1}=0.048$, Mach number $M_{a}=1.67$.

test sections shown in the same figure. These images are compared with the numerical results of FLASH software. Figure 5.2 shows the comparisons between three different histories (time steps)of interface development experimentally and numerically for first case $\left(A_{e 1}=0.048\right)$.

Good agreement can be seen in theses histories by comparing the distances between the deposited vortices centers in first and second rows, as shown in the same figure. In general, These distances are growing up due to the effect of compressibility and SDMI generation. The main reason that shows big particles lagging behind small particles is that the mass of big particles have long momentum relaxation than those 
of small size. One can calculate momentum relaxation time using the following[23].

$$
\tau_{v}=\frac{m_{p}}{6 \pi r_{p} \mu_{g}}
$$

Equation 5.1 shows that momentum relaxation time of particle proportional with the radius square. Thermal relaxation time $\left(\tau_{T}\right)$, Equation 5.2, however will be larger than the momentum relaxation time $\left(\tau_{\nu}\right)$. Since $\left(\tau_{\nu}<\tau_{T}\right)$ and time of simulation is small $(t=4 m s)$, The author chooses not to include phase change in the simulation to accomplish the comparisons.

$$
\tau_{T}=\frac{m_{p} C_{p}}{4 \pi r_{p} K_{g}}
$$

some differences are noticed in the Figure 5.2. These differences comes from some facts.

1. The Particles have random nature, Random theory is always related to statistical errors.

2. We consider two-dimensional problem, however, the experimental results gives us a snap shot of 3D illuminated layer of interface.

3. The picture we get from experimental results is an interaction of light with small particles, these particles scatter, absorb and re-emit lights which gives kind of foggy picture.

4. Break up model,condensation, or evaporation were not considered due their slight effect as we mentioned earlier.

5. Boundary conditions are set on the test section, this will make a slight effects on the interface development as well. 

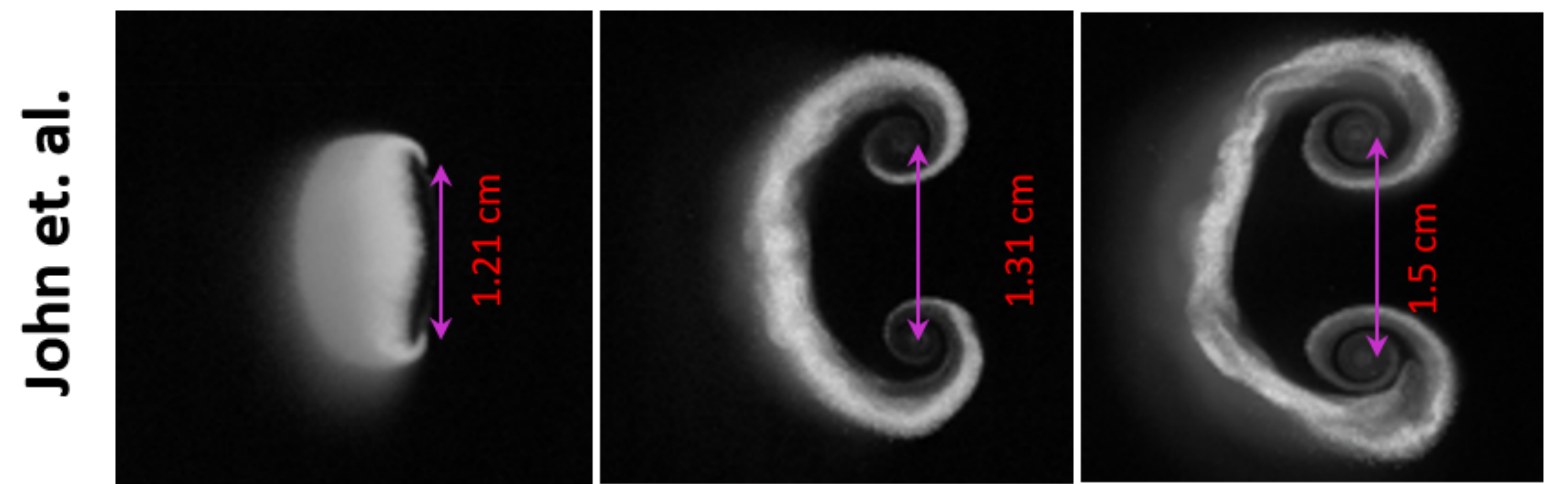

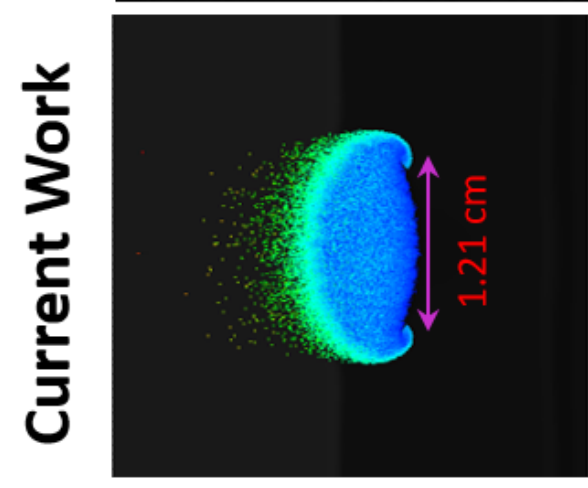

Time $=0.5 \mathrm{~ms}$

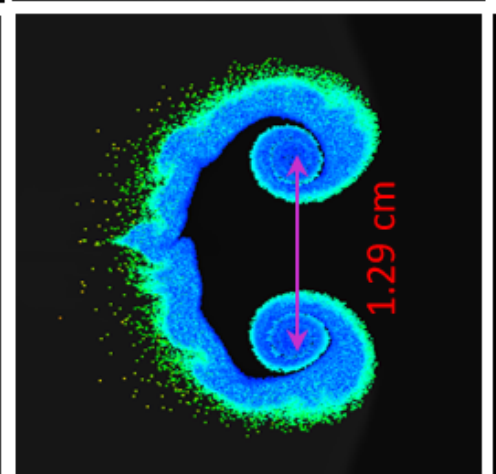

Time=2.9 $\mathrm{ms}$

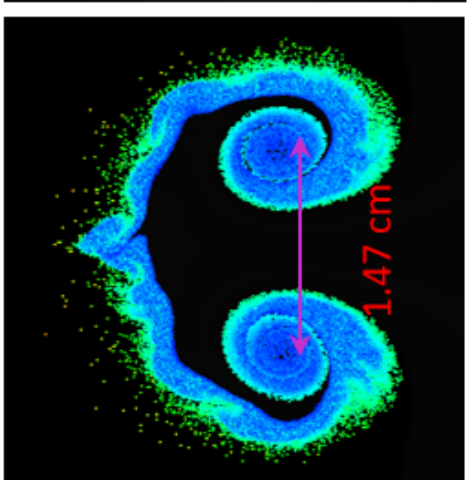

Time=4 ms

Figure 5.3: Comparisons of SDMI mosaic between experimental results, first Row and numerical solution second row for particle distribution parameters with , $\mu_{0}=1.7 \mu \mathrm{m}$ and $\sigma_{0}=0.728 \mu \mathrm{m}$ and Atwood number $A_{e 2}=0.0184$, Mach number $M_{a}=1.67$.

Figure 5.3 shows the comparisons between experimental and numerical solutions when Atwood number $\left(A_{e 2}=0.0184\right)$. One interesting difference is noticed between Figure $5.2 \& 5.3$ is that SDMI is stronger when Atwood number is higher.

When Atwood gets bigger, the development happens faster, and the deposited vortex tries to rap all the interface making the crescent-like shape side of the interface to be thinner. However, when the Atwood number gets smaller, the distances between the vortexes center get bigger compared with high Atwood number one. The crescentlike shape in at $t=0.5$, which formed from lagging big particles, looks smooth, and then it gets deformed because of the weak vortex generated in the low Atwood number case. 
To sum all above, The Atwood number is critical parameter that controls the SDMI instability, also the drag on particles will have also a great effect as well. We have mentioned that the as the size (increase the mass) of the particle increases, the relaxation time increases. However, increasing the size will also increase the drag force. As a result, drag, size of particles, Atwood number are controlling this phenomena.

\subsection{Simulation of AGB Star Problem}

In this section, we will be exploring some observations results to see how these observations close or far from the simulation we have done. The simulation section will go through the histories of SDMIs' evolutions near AGB star.

\subsubsection{AGB stars Observations}

Agb star envelops evolutions come from two critical processes. Chemical and physical processes, Those two are working together, causing many phenomena. Dust formation, shock-induced chemistry, as well as, photo-dissociation due to cosmic rays and UV photons coming from outer space are all consequences of these processes[53][54]. Infrared observation discloses many secrets about AGB stars. IRAS (Infrared Astronomical Satellite) noticed low-temperature circumstellar dust (above convection envelop, see Figure 1.7)[55]. At this envelop, dust is formed from the hot gases through condensation process. Shocks are triggered by pulsating motions of AGB star convection envelope, These shocks will alleviate the high density gases into the cold dust formation region at which dust is formed by condensation processes, but this is still puzzling processes. Dust absorbs near UV lights and emits strongly at mid- to far-infrared wave lengths, so in the following observations two wave lengths are considered $(70 \mu m, 160 \mu m)$. 

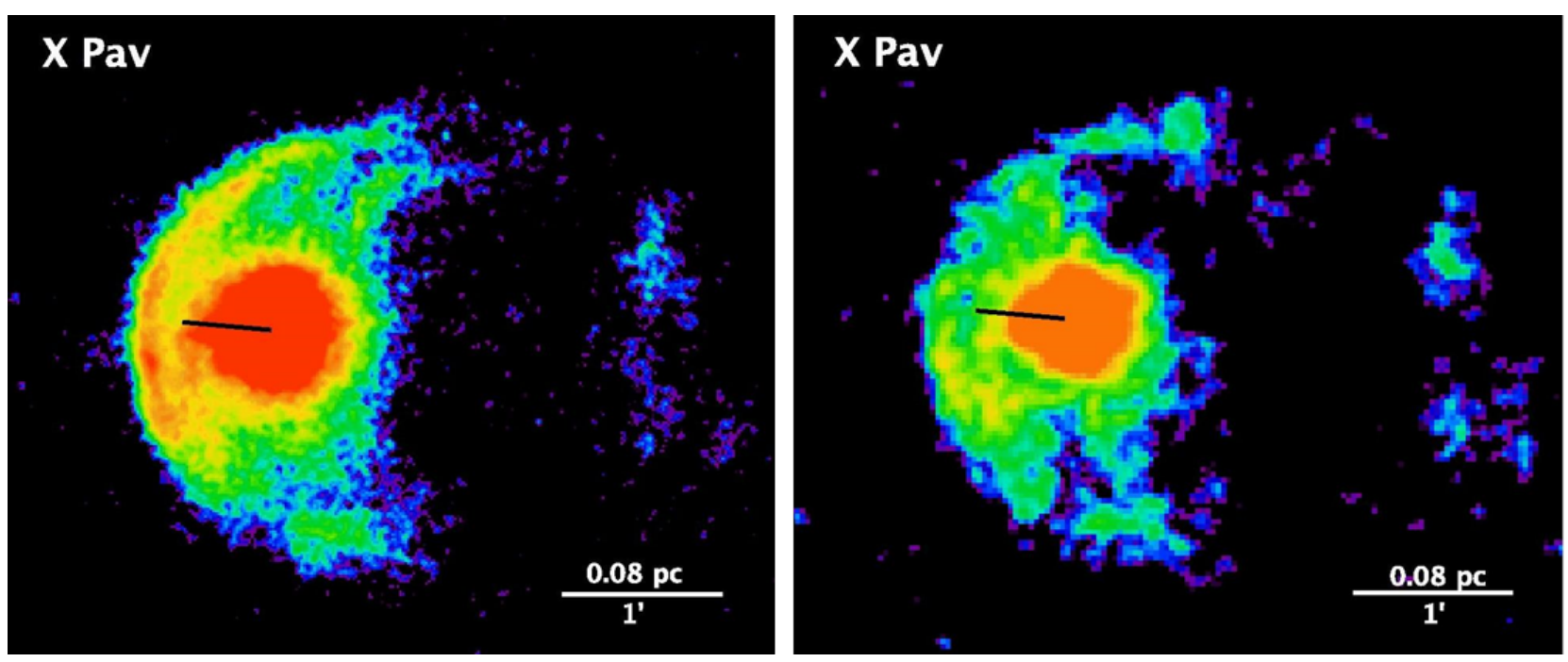

Figure 5.4: Herschel/PACS images at $70 \mu m$ (left) and $160 \mu m$ (Right), X Pav object $[50]$.

Figure 5.4 presents Herschel/PACS telescopes images at mid- and far-infrared spectra. These two images show clearly big arcs spanning $90^{\circ}$ degrees. Those arcs (detached bow shocks) are the interactions between cold dust with interstellar medium. This bow shocks are created when supersonic stellar wind collide cold interstellar medium. Cold ISM can create shocks easily $\left(M_{a}=v_{\text {wind }} / \sqrt{\gamma R T_{\text {cold }}}\right)$. So when temperature goes down the velocity of sound goes down and Mach goes up. The solid line on the image refers to the direction of the star from the center of the star to the outer space. The right image in Figure 5.4 shows blobby shape like of circumstellar medium. This region is not stable and SDMI is significant at CSM while KHI and RTI can be seen clearly in the ISM[11][46][50]. One more interesting morphology which barely can be seen in far-infrared image is the eye like shapes, see yellow spots in CSM in right image. These Double non-concentric arcs are clearly seen in the Figure 5.5 .

Figure 5.5 shows that most of these eyes has elliptic morphology. These eyes are injected from CSM due to bubble shock effects as we will see in simulation section. Wind-blown bubble in interstellar medium is rarely seen [56]. To search for 

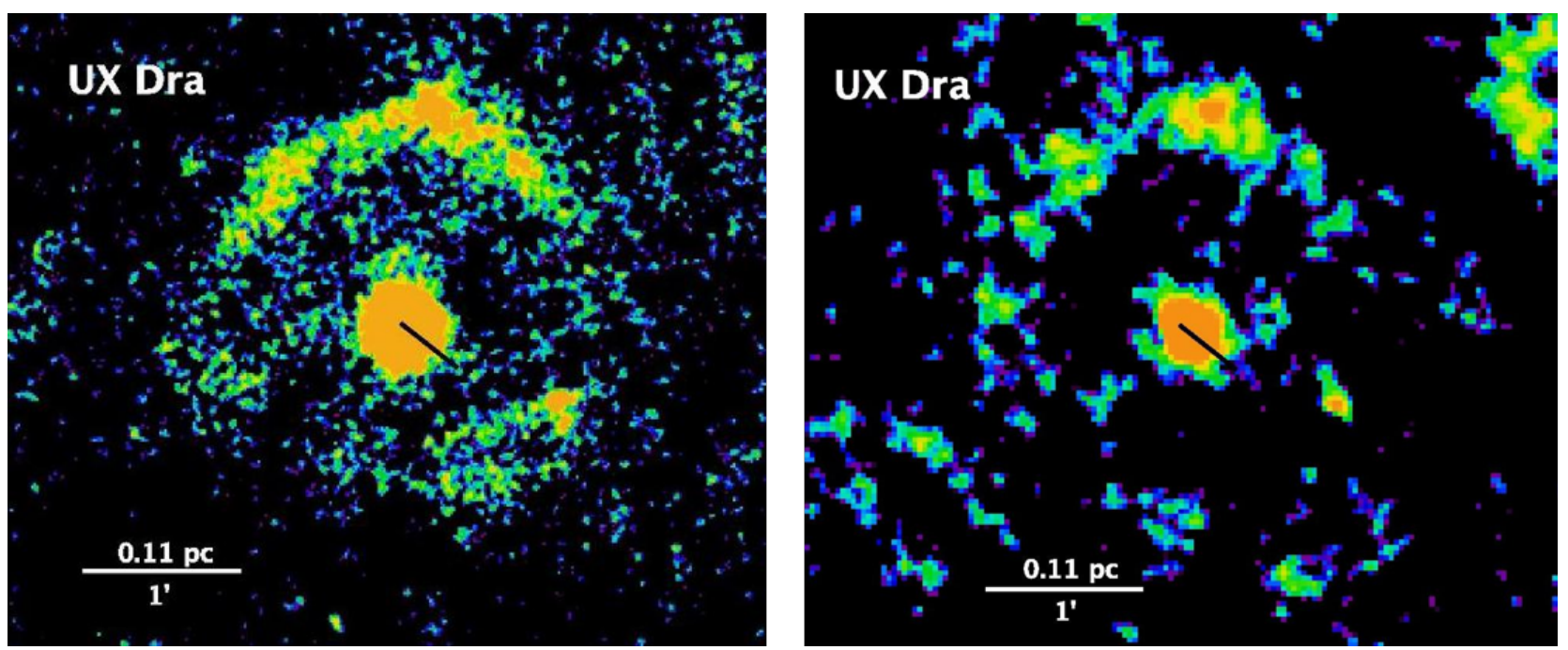

Figure 5.5: Herschel/PACS images at $70 \mu m$ (left) and $160 \mu m$ (Right), UX Dra object [50].

wind-blown bubble, Astronomers should use high resolution Hubble Space Telescope (HTS)[55]. SDMI generated in CSM due to shock wave interaction dust is leading to these bubbles before they merge into ISM.

Figures 5.6 shows the a complete ring encircling the CSM of the star. The rings are always having $360^{\circ}$ spanning angle, and they have spherical shape or oval-like shape. This shape includes detached dust shell and bow shock we noticed in X Pav. Again, The blobby shape can be seen clearly at the center of the U Ant object.

To sum all morphology's observation we have addressed. There are four large scale dust shapes are expected as follow.

1. Arcs, These are large scale arc structure, or shell-like structure with an angle $90^{\circ}$. These arcs are generated due to CSM and ISM interaction, see Figure 5.3.

2. Eyes, Two arcs making an elliptic shape, see Figure 5.4.

3. Rings, These objects have spherical or oval-like. shape,see Figure 5.5.

4. Irregular, this morphology has no specific shape and it can be seen in all Figures. 

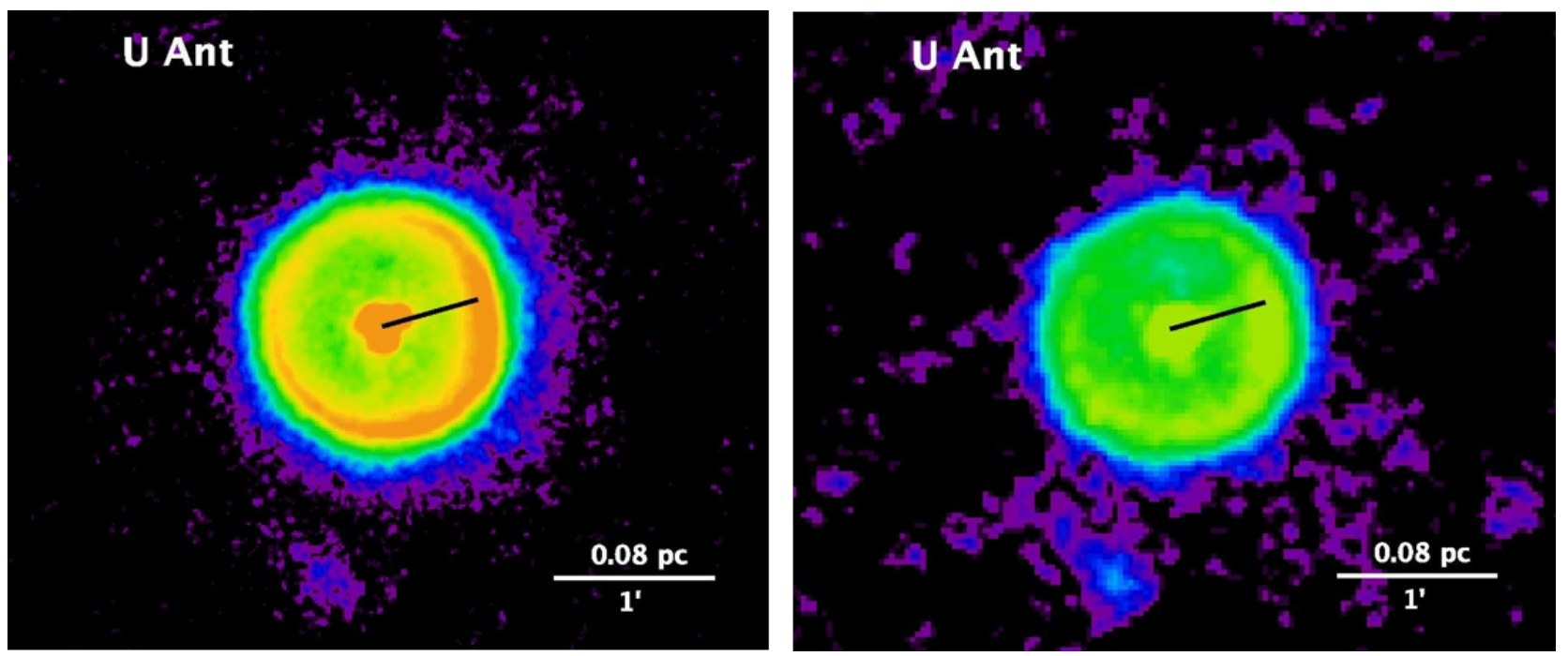

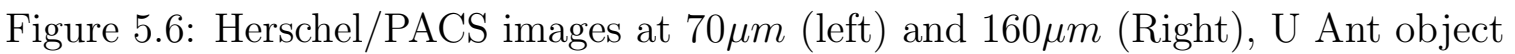
[50].

Those shapes generally are consequences of physical and chemical processes that occurs in convection and CSM. In the next section, we will be going through simulation part of AGB star.

\subsection{SDMI Simulation near AGB star}

From previous section and Figure 1.7, It is clear that the dust has formed at a radial position $R$ where $\left(R_{\text {star }}<R<10 R_{\text {star }}\right)$. In the current study, I assumed that the radius of the star $\left(R_{\text {star }}=0.25 \times 10^{14} \mathrm{~cm}\right)$ with single dust shell at radius $\left(0.7 \times 10^{14} \mathrm{~cm}\right)$ and thickness $\left(0.15 \times 10^{14} \mathrm{~cm}\right)$. We will be exploring the development of this dust shell with time to see all expected morphologies. Due to complexity of physical and chemical processes, many assumption are considered in this simulation.

By recalling SDMI ingredients in chapter one, we have to have a shock wave interacting with the dust at CSM, where dust is observed first. To do so, one might consider a compressed or high pressure region in the star to generate some shocks that expand through CSM encountering the dust. This assumption even seems to be 


\begin{tabular}{|cccccc|}
\hline Domain & Time step & No. of Blocks & Gamma & B.Cs. & Star Temp. \\
\hline $7.5 e 14 \times 7.5 e 14 \mathrm{~cm}$ & $2.0 \times 10^{6} \mathrm{sec}$ & $120 \times 120$ & 1.41 & OutFlow & $2500 \mathrm{~K}$ \\
\hline
\end{tabular}

Table 5.2: Main Inputs in AGB star problem

reasonable due single shock generation, but one might argue that we are simulating an exploded star which is not the real case. To resolve this argument, we can impose a constant initial condition that can give us continuous shocks to fit with the real situation at convection envelope. To do so, there are many scenarios can be followed. The scenario, that I implement in our code, is to consider the a region of star $(R \leq$ $R_{\text {star }}$ ) with constant properties to empower continuous shock generation in CSM. This step will improve the simulation to give reasonable solution. After we set the problem as we mentioned earlier, A simulation is run for specific inputs parameters, see table 5.2, and for more details, the FLASH input parameters file is included in Appendix A.5. 

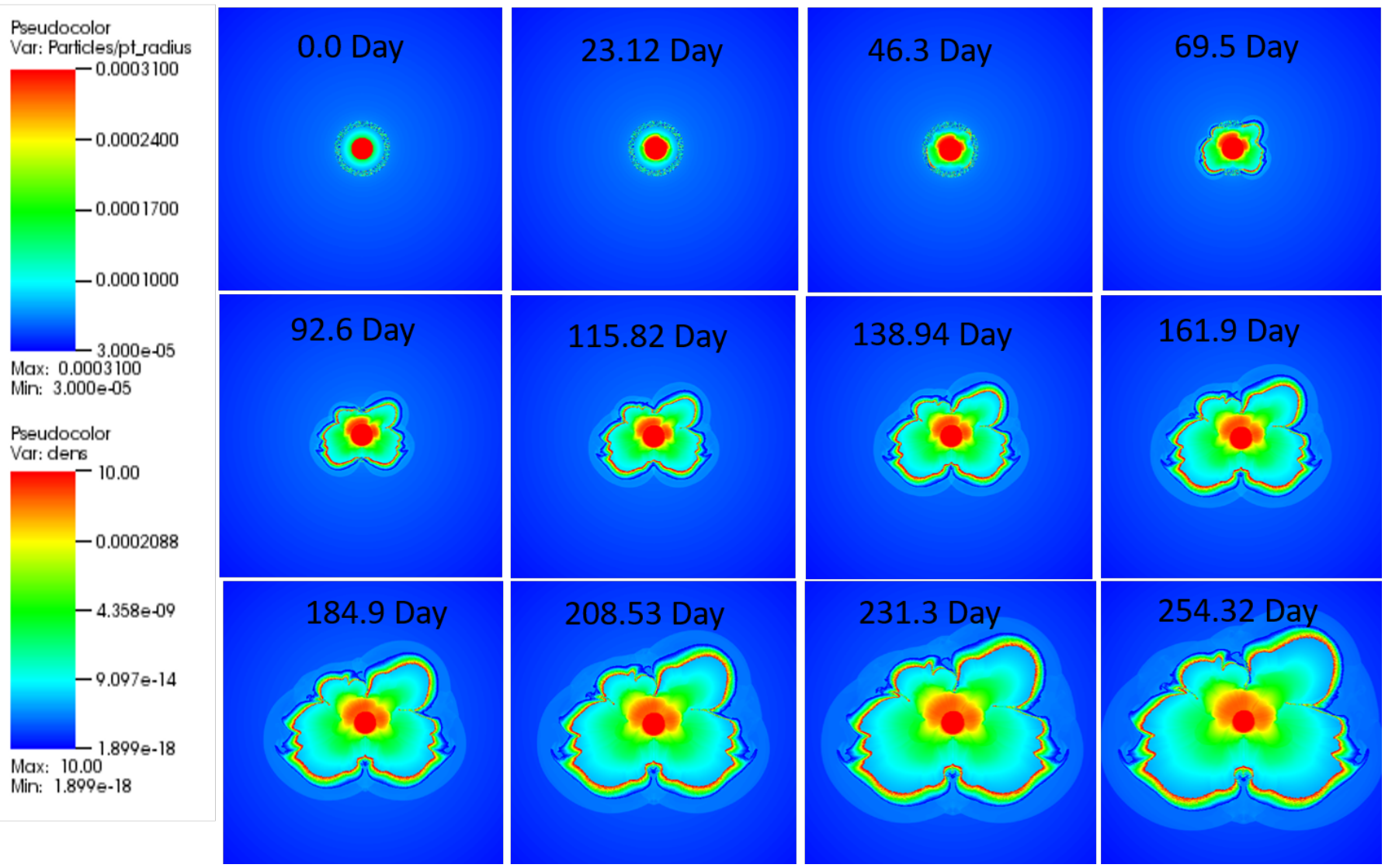

Figure 5.7: One Dust Shell Development driven by Bubble shock, and radiation.

Figure 5.7 shows density histories near AGB star, when $(t=0.0$ Day $)$ density of gas distribution has an exponential decay, which is explained in previous section and shown in Figure 4.6. At $t=23.12$ Day $\left(2.0 \times 10^{6} \mathrm{sec}\right)$ the star launch first bubble shocks (hot gases) that expands in outer space pushing the dust with the help of radiation. This part of figure simulates production of shocks triggered by pulsating and chemical processes in real situation even we didn't consider these processes. When $t=46.3$ Day , density increases due to star mass loss and (only gas for now which some of it turns to dust due to condensation processes at $R>R_{\text {star }}$ ). First morphology noticed here is the blobby-like shape, see yellowish green area around the AGB star. Dust shell, in first two time steps, has no interaction with moving shocks yet. Further more, countour plot of dust reflects the log-normal distribution shown in Figure 4.9A. 
Other interesting picture we can see at time $t=46.3$ is a small red arcs at the front of shocks, where first interactions between shocks and dust happens. These arcs are reflects lagging of big size particles behind small ones. This, in fact, shows the first interesting sign of SDMI generation. SDMI's morphology has no limited shape near AGB star. Since our domain is large, we will address large scale morphologies.

The shocks go far towards CSM supported by star processes (source of energy) by continuous bubble shocks generations. Small particles follows the gas flow as a flow tracers showing the second feature of SDMI and big particles lag behind. This also shows that the implemented non-continuum drag model works fine in this study. In this simulation we didn't consider the interactions between ISM and CSM,for example, every star has specific motion as we saw in Figures 5.6,5.5, \& 5.4.

The gas and dust continue to expand for the rest of time steps, and all early formed morphologies grow up making big arcs. These arcs are eventually interacting with ISM to develop bow shocks shown in the observations, see Figure 1.7. Our simulation doesn't reach far till $R>10000 R_{\text {star }}$ where ISM radiation and cold conditions effecting on CSM. If we want to go that far we have to have very high computational power which is not available at HPC in MIZZOU (at least 10,000 $\times 10,000$ blocks, $10^{5}-10^{7}$ $\mathrm{cpu})$.

One might ask the following question "Why are we seeing asymmetric behaviour of gas and, while we assume most initial condition are symmetrical?", to answer this question let us go to the next section 5.4.1.

\subsubsection{Radiation and gravity effects}

The gravity acceleration attracts the dust and gas towards the star, while radiation push on opposite direction. The gravity model we implement on the gas and particles is the point mass model,section 3.3, has less effects than the radiation acceleration. If we do our calculation at the dust shell, we will find that the ratio of radiation to 
gravity acceleration is $\sim 1500$ times. However, Accelerated gas from the star is more significant than radiation acceleration due to continuous shocks generation because of complex physical and chemical processes mentioned earlier.

One might ask a question " Why don't we see the effects of the Radiation in the simulation?". To answer this question, let us make a link between this question and the previous question at last paragraph, section 5.4. The Answer is radiation simply is there and which cause asymmetric behaviour to the properties around the star. The Perturbed radiation field shown in Figure 4.7 with have essential effects in asymmetric conditions on both gas and dust because all our previous initial conditions were symmetric and the developed shocks shuld be in this case a continuous blast wave instead of bubble wave. For more details about gravity model implementation, see Appendix A.4. 


\section{Chapter 6}

\section{Conclusions and Suggestion for Future Works}

In this study, tow tasks are accomplished. The first task of the current research is done by comparing experimental and numerical solutions of interface evolutions on shock tube facility at the University of Missouri at Columbia. The second task is extending code capabilities to deal with the dust evolving near AGB stars.

Both problems are solved using E-L (MP-PIC) systems of equations with different physics. For the second problem, new radiation, Gravity, and non-continuum model to the implemented code. In this method, Eulerian gas flow and Lagrangian particles cloud are solved separately; however, source terms are added to the Eulerian system using a mapping from particle location to the gas mesh and vise-versa.

Particles are generated randomly in both cases following log-normal size distribution and uniform location distribution. A discrete parcel method is used for both problems instead of the discrete element method. Two different simulations runs are considered in the shock tube problem, and one simulation is discussed near the AGB star problem.

To draw a picture of the conclusions of the SDMI validation and extension in the 
AGB star problem, The following bullets are going to summarize them.

- Fair comparisons were done between Experimental and numerical results qualitatively, and quantitatively,also, There is a agreement between the shape and development of the interface at different time steps. SDMI problem is too sensitive to the initial and boundary conditions due to the fact that dealing with different scales at same time.

- SDMI problem involves with many physics and we should make a priority to deal with the physics.

- Particle properties distributions is the main factor controlling SDMI development.

- SDMI near AGB star is complex and puzzling phenomena.

- Complexity of SDMI near AGB star comes from different envelopes that have different physics and different couplings, with multispecies, pulsating motions, shock waves, nuclear fusions etc.

- It is hard to resolve small scale instability structures within large scale in AGB stars due to high levels of refinements needed in such cases.

HIs near AGB stars include all types of flow instabilities known, That encourage scientist to study these huge objects in different levels of complexities. One might consider RTI and KHI at far distance in outer-space and/or SDMI near AGB star. Making such decision rely on the physics and chemist we need to include. This will open the doors for many future works. My suggestion for further works can be listed as follow.

1. Extend This work to three Dimensional simulation.

2. Consider dimensional and spectral Badiation parameters. 
3. Time rate of dust generation rather than one or multiple dust shells is necessary for full dynamics of dust.

4. Including pulsating conditions to see how that effects shock generations.

5. Considering chemical reaction in convection envelops. 


\section{Bibliography}

[1] Philip G Drazin. Introduction to hydrodynamic stability, volume 32. Cambridge university press, 2002.

[2] Houjun Mo, Frank Van den Bosch, and Simon White. Galaxy formation and evolution. Cambridge University Press, 2010.

[3] John I Castor. Radiation hydrodynamics. Cambridge University Press, 2004.

[4] François Charru. Hydrodynamic instabilities, volume 37. Cambridge University Press, 2011.

[5] Ye Zhou. Rayleigh-taylor and richtmyer-meshkov instability induced flow, turbulence, and mixing. ii. Physics Reports, 723:1-160, 2017.

[6] Baltimore, Maryland, USA,Space Telescope Science Institute. Crab nebula image, 2013. [Online; accessed April 27, 2019].

[7] Aeronautics and Space Administration (NASA). Sarychev volcano, 2002. [Online; accessed April 28, 2019].

[8] Martin Brouillette. The richtmyer-meshkov instability. Annual Review of Fluid Mechanics, 34(1):445-468, 2002. 
[9] Allard Jan van Marle, Zakaria Meliani, Rony Keppens, and Leen Decin. Computing the dust distribution in the bow shock of a fast-moving, evolved star. The Astrophysical Journal Letters, 734(2):L26, 2011.

[10] Leen Decin. Late stages of stellar evolution-herschel's contributions. Advances in Space Research, 50(7):843-863, 2012.

[11] Peter Woitke. Dust-driven winds beyond spherical symmetry. Proceedings of the International Astronomical Union, 4(S252):229-234, 2008.

[12] Susanne Höfner. Winds of m-type agb stars driven by micron-sized grains. Astronomy \& Astrophysics, 491(2):L1-L4, 2008.

[13] S. Bladh, S. Höfner, B. Aringer, and K. Eriksson. Exploring wind-driving dust species in cool luminous giants. III. Wind models for M-type AGB stars: dynamic and photometric properties., 575:A105, March 2015.

[14] Hans-Peter Gail and Erwin Sedlmayr. Physics and chemistry of circumstellar dust shells, volume 52. Cambridge University Press, 2014.

[15] Susanne Höfner and Hans Olofsson. Mass loss of stars on the asymptotic giant branch. The Astronomy and Astrophysics Review, 26(1):1, 2018.

[16] Clayton T Crowe, John D Schwarzkopf, Martin Sommerfeld, and Yutaka Tsuji. Multiphase flows with droplets and particles. CRC press, 2011.

[17] Said Elghobashi. On predicting particle-laden turbulent flows. Applied scientific research, 52(4):309-329, 1994.

[18] Michael J Andrews and Peter J O'Rourke. The multiphase particle-in-cell (mppic) method for dense particulate flows. International Journal of Multiphase Flow, 22(2):379-402, 1996. 
[19] Meiyan Feng, Fei Li, Wei Wang, and Jinghai Li. Parametric study for mp-pic simulation of bubbling fluidized beds with geldart a particles. Powder technology, $328: 215-226,2018$.

[20] DM Snider, PJ ORourke, and MJ Andrews. An incompressible two-dimensional multiphase particle-in-cell model for dense particle flows. Technical report, Los Alamos National Lab., NM (United States), 1997.

[21] DM Snider. An incompressible three-dimensional multiphase particle-in-cell model for dense particle flows. Journal of computational physics, 170(2):523$549,2001$.

[22] Jacob A McFarland, Wolfgang J Black, Jeevan Dahal, and Brandon E Morgan. Computational study of the shock driven instability of a multiphase particle-gas system. Physics of Fluids, 28(2):024105, 2016.

[23] Jeevan Dahal and Jacob A McFarland. A numerical method for shock driven multiphase flow with evaporating particles. Journal of Computational Physics, 344:210-233, 2017.

[24] CPFD Software. Llc. barracuda 14.4 released, 2011. [Online; accessed Jan,10,2020].

[25] L OpenFOAM. User guide version 4.0, the openfoam foundation. 2016.

[26] ASC FLASH Center. Flash user's guide. University of Chicago: Chicago, 2005.

[27] Jeffrey W Jacobs, VV Krivets, V Tsiklashvili, and OA Likhachev. Experiments on the richtmyer-meshkov instability with an imposed, random initial perturbation. Shock Waves, 23(4):407-413, 2013.

[28] BJ Balakumar, GC Orlicz, JR Ristorcelli, S Balasubramanian, KP Prestridge, and CD Tomkins. Turbulent mixing in a richtmyer-meshkov fluid layer after 
reshock: velocity and density statistics. Journal of fluid mechanics, 696:67-93, 2012.

[29] David Reilly, Jacob McFarland, Mohammad Mohaghar, and Devesh Ranjan. The effects of initial conditions and circulation deposition on the inclined-interface reshocked richtmyer-meshkov instability. Experiments in Fluids, 56(8):168, 2015.

[30] Jacob McFarland, David Reilly, Skylar Creel, Christopher McDonald, Thomas Finn, and Devesh Ranjan. Experimental investigation of the inclined interface richtmyer-meshkov instability before and after reshock. Experiments in fluids, $55(1): 1640,2014$.

[31] Peter Vorobieff, Michael Anderson, Joseph Conroy, Ross White, C Randall Truman, and Sanjay Kumar. Vortex formation in a shock-accelerated gas induced by particle seeding. Physical review letters, 106(18):184503, 2011.

[32] M Anderson, Peter Vorobieff, CR Truman, C Corbin, G Kuehner, P Wayne, J Conroy, R White, and S Kumar. An experimental and numerical study of shock interaction with a gas column seeded with droplets. Shock Waves, 25(2):107-125, 2015.

[33] John B Middlebrooks, Constantine G Avgoustopoulos, Wolfgang J Black, Roy C Allen, and Jacob A McFarland. Droplet and multiphase effects in a shock-driven hydrodynamic instability with reshock. Experiments in Fluids, 59(6):98, 2018.

[34] Erwin Sedlmayr and Carsten Dominik. Dust Driven Winds. , 73(3-4):211-272, August 1995.

[35] Bernd Freytag and Susanne Höfner. Three-dimensional simulations of the atmosphere of an agb star. Astronomy \& Astrophysics, 483(2):571-583, 2008. 
[36] Bernd Freytag, Sofie Liljegren, and Susanne Höfner. Global 3d radiationhydrodynamics models of agb stars-effects of convection and radial pulsations on atmospheric structures. Astronomy \& Astrophysics, 600:A137, 2017.

[37] John David Anderson. Modern compressible flow: with historical perspective, volume 12. McGraw-Hill New York, 1990.

[38] Hermann Schlichting and Klaus Gersten. Boundary-layer theory. Springer, 2016.

[39] Satish Kandlikar, Srinivas Garimella, Dongqing Li, Stephane Colin, and Michael R King. Heat transfer and fluid flow in minichannels and microchannels. elsevier, 2005.

[40] T Bergman, A Lavine, FP Incropera, and DP Dewitt. Fundamentals of Heat and Mass Transfer, volume 201609. John Wiley \& Sons, 2016.

[41] A Chafiq and A Belafhal. Radiation pressure cross section exerted on homogenous dielectric spherical particle by zeroth order mathieu beams. Journal of Quantitative Spectroscopy and Radiative Transfer, 179:170-176, 2016.

[42] John R Howell, M Pinar Menguc, and Robert Siegel. Thermal radiation heat transfer. CRC press, 2016.

[43] Hiroshi Kimura and Ingrid Mann. Radiation pressure cross section for fluffy aggregates. Journal of Quantitative Spectroscopy and Radiative Transfer, 60(3):425-438, 1998.

[44] Oliver Krauß and Gerhard Wurm. Radiation pressure forces on individual micron-size dust particles: a new experimental approach. Journal of Quantitative Spectroscopy and Radiative Transfer, 89(1-4):179-189, 2004.

[45] Ashok Kumar. Guidelines for evaluating the characteristics of vapor cloud explosions, flash fires, and bleves. center for chemical process safety (ccps) of the aiche, 
published by the american institute of chemical engineers, new york, ny (1994), 387 pages,[isbn: 0-8169-0474-x], us list price: 150. Environmental Progress, 15(1):S11-S12, 1996.

[46] Peter Woitke. 2d models for dust-driven agb star winds. Astronomy 83 Astrophysics, 452(2):537-549, 2006.

[47] Bruce Fryxell, Kevin Olson, Paul Ricker, FX Timmes, Michael Zingale, DQ Lamb, Peter MacNeice, Robert Rosner, JW Truran, and H Tufo. Flash: An adaptive mesh hydrodynamics code for modeling astrophysical thermonuclear flashes. The Astrophysical Journal Supplement Series, 131(1):273, 2000.

[48] Jeevan Dahal and Jacob A. McFarland. A numerical method for shock driven multiphase flow with evaporating particles. Journal of Computational Physics, 344:210-233, September 2017.

[49] JV Sengers, Y-Y Lin Wang, B Kamgar-Parsi, and JR Dorfman. Kinetic theory of drag on objects in nearly free molecular flow. Physica A: Statistical Mechanics and its Applications, 413:409-425, 2014.

[50] NLJ Cox, F Kerschbaum, A-J van Marle, Leen Decin, D Ladjal, A Mayer, MAT Groenewegen, S van Eck, Pierre Royer, R Ottensamer, et al. A far-infrared survey of bow shocks and detached shells around agb stars and red supergiants. Astronomy \&3 Astrophysics, 537:A35, 2012.

[51] A. J. Fleischer, A. Gauger, and E. Sedlmayr. Circumstellar dust shells around long-period variables. III. Instability due to an exterior $\kappa$-mechanism caused by dust formation., 297:543, May 1995.

[52] John S Mathis. Interstellar dust and extinction. Annual Review of Astronomy and Astrophysics, 28(1):37-70, 1990. 
[53] K Willacy and TJ Millar. Chemistry in oxygen-rich circumstellar envelopes. Astronomy and Astrophysics, 324:237-248, 1997.

[54] Leen Decin, Marcelino Agúndez, Michael J Barlow, Fabien Daniel, José Cernicharo, Robin Lombaert, Elvire De Beck, Pierre Royer, Bart Vandenbussche, $\mathrm{R}$ Wesson, et al. Warm water vapour in the sooty outflow from a luminous carbon star. Nature, 467(7311):64-67, 2010.

[55] WECJ Van der Veen, HJ Habing, et al. The iras two-colour diagram as a tool for studying late stages of stellar evolution. Astronomy and Astrophysics, 194:125, 1988.

[56] Sarah Jane Arthur. What produces the diffuse x-ray emission from the orion nebula? i. simple spherical models. Monthly Notices of the Royal Astronomical Society, 421(2):1283-1297, 2012. 


\section{Appendix A}

\section{Codes Implemented}

\section{A.1 One Dimensional Shock tube Analysis}

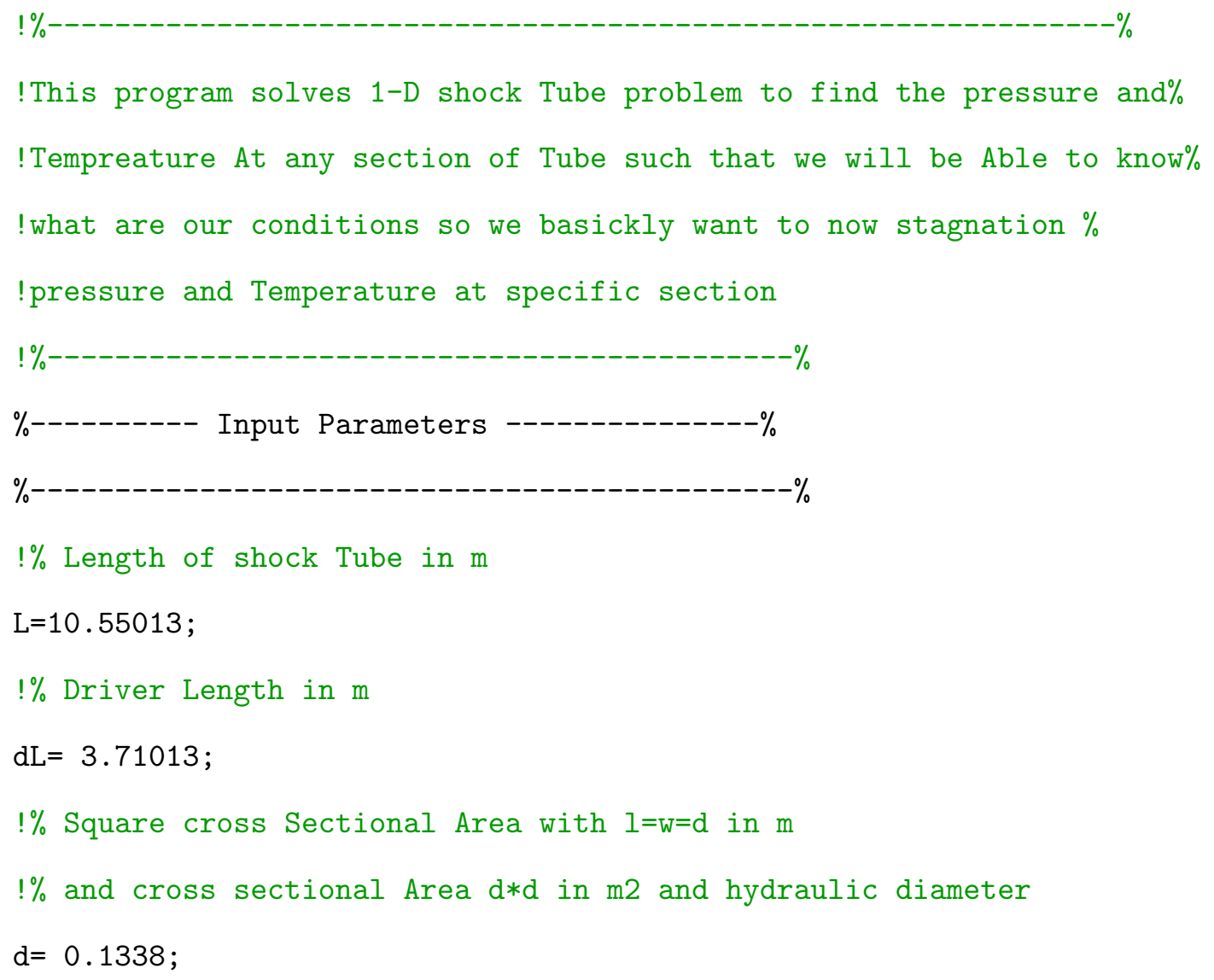




\section{$\mathrm{As}=\mathrm{d} * \mathrm{~d}$}

$\mathrm{hD}=4 * \mathrm{As} /(\mathrm{d}+\mathrm{d}+\mathrm{d}+\mathrm{d}) ; ! \%$ same as $\mathrm{d}$ for sure

!\% properties of working fluid so we have either dry Air

!\% or $\mathrm{N} 2$ inside the driver section however we have missed

! $\%$ air(water vapor with $\% 15$ relative humidity) and N2

!\% $\mathrm{T}$ in $\mathrm{K}$ and pressure in pascal each 1 psia 6894.75728 pascal

!\% pE expansion section pressure which is $101325 \mathrm{pa}$

$\mathrm{T}=300 ; \mathrm{pD}=100 * 6894.75728 ; \mathrm{pE}=101325$;

$\mathrm{pR}=11.2276 ; ! \% \mathrm{pD} / \mathrm{pE}$;

$! \%$ if we have pressure ratio we can get Mach Number in stagnent flow and in !\% moving flow region by considering moving frame(say applying governing

$! \%$ equation in moving control volume such that the stagnent fluid will have !\% a velocity equals to moving shock speed (Us) so lets find the properties $! \%$ of all variables considering gas dynamics table.

!\%gas dynamic Table parameter for normal shock wave for moving shock

!\%it will stay same equations except

gama $=1.4$

$\mathrm{n}=30 ; \operatorname{Mmax}=4.0 ; \operatorname{Mmin}=1.0$;

Mx $=$ linspace $(\operatorname{Mmin}, \operatorname{Mmax}, \mathrm{n})$;

$\mathrm{PyPx}=1+2 *$ gama $/($ gama +1$) *\left(\mathrm{Mx} \cdot{ }^{\wedge} 2-1\right)$;

My=sqrt $\left(\left(\right.\right.$ Mx $\cdot{ }^{\wedge} 2+2 /($ gama-1) $) . /\left(2 *\right.$ gama $/\left(\right.$ gama-1) $*$ Mx $\left.\left.\cdot{ }^{\wedge} 2-1\right)\right)$;

RhoyRhox $=\left((\right.$ gama +1$\left.) * M x \cdot{ }^{\wedge} 2\right) . /\left(2+(\right.$ gama -1$\left.) * M x \cdot{ }^{\wedge} 2\right)$;

$\mathrm{TyTx}=\left(1+(\right.$ gama-1) $\left.) / 2 * \mathrm{Mx} \cdot{ }^{\wedge} 2\right) . *\left(2 *\right.$ gama $/($ gama -1$\left.) * \mathrm{Mx} \cdot{ }^{\wedge} 2-1\right) . /$

$\left((\text { gama }+1)^{\wedge} 2 /(2 *(\right.$ gama -1$\left.)) * M x \cdot{ }^{\wedge} 2\right)$;

!\%Lets start solve the problem

!\% in calculations so basickly the normal one is the only one we can

!\%consider here which means we can only change $\mathrm{M}$ in above equations

!\% with Mn1 also there is a relation between theta, beta and M1

!\% say Mx that coluld ploted as follow. 


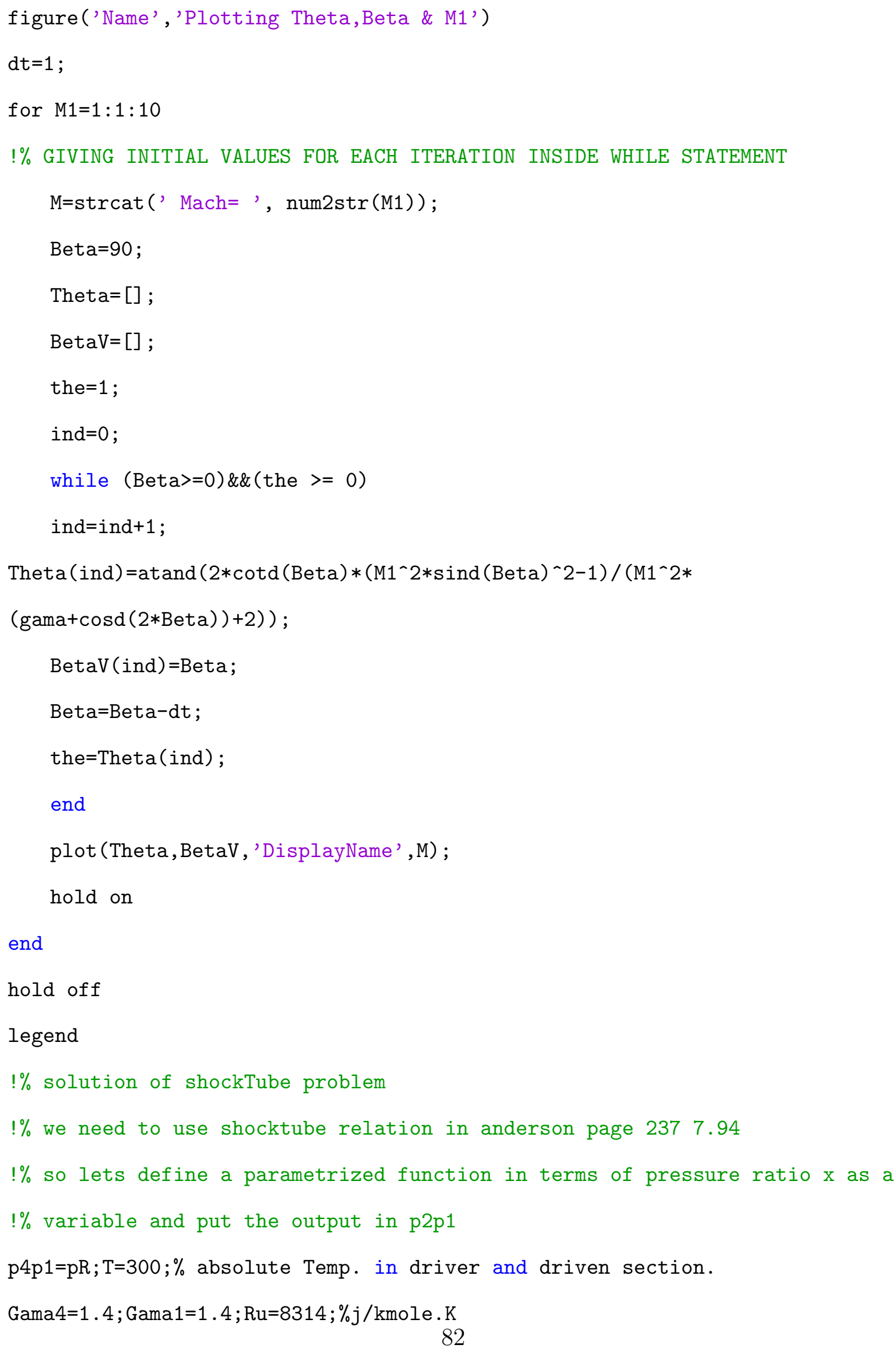




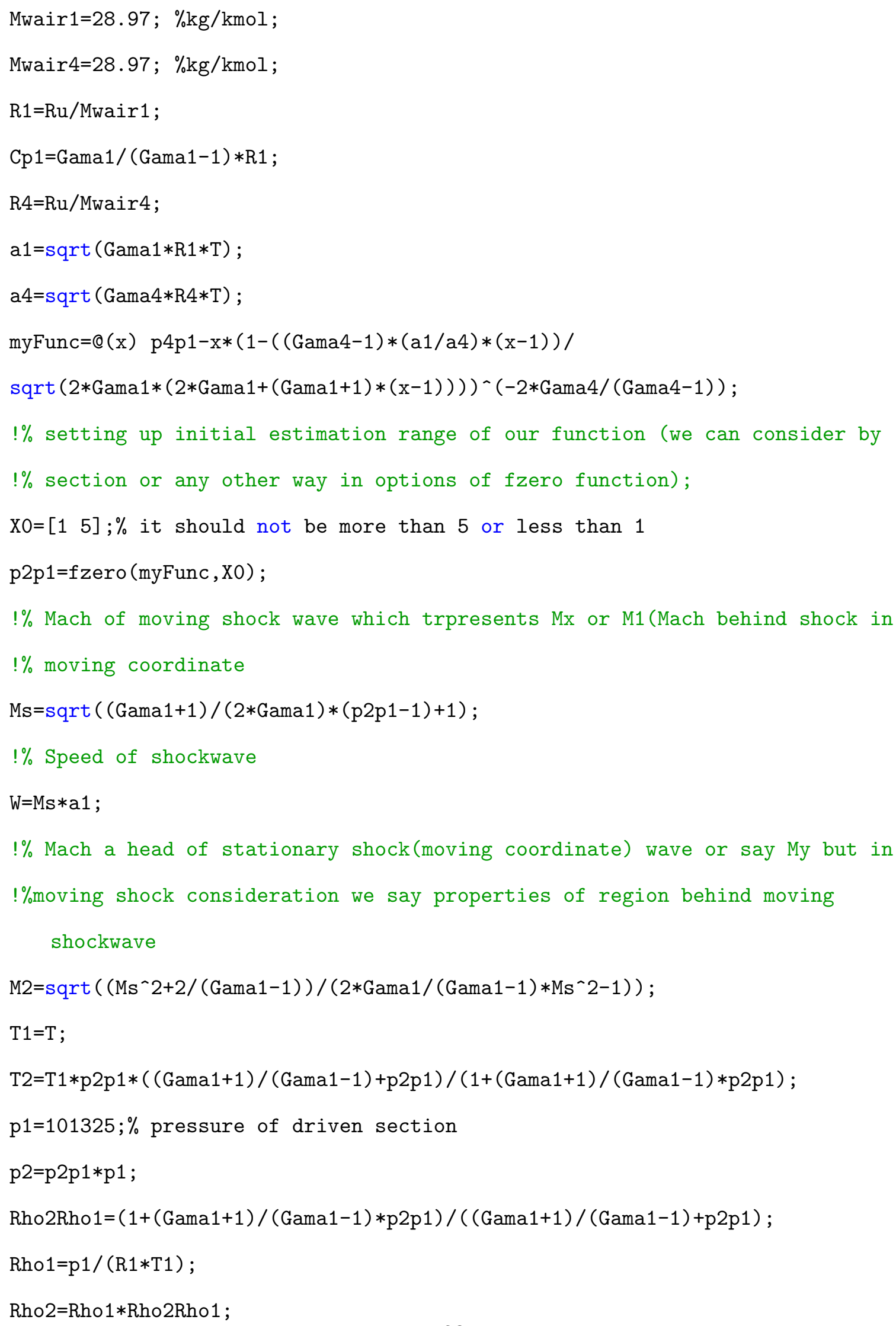


!\% speed of flow behind the shock Region 2

$\mathrm{Up}=\mathrm{W} *(1-1 / \mathrm{Rho} 2 \mathrm{Rh} 01)$;

fprintf('shock Mach= \%10.3f m/s $\backslash \mathrm{n}$ ',Ms);

fprintf('shock speed= $\% 10.3 \mathrm{f} \mathrm{m} / \mathrm{s} \backslash \mathrm{n}$ ',W);

fprintf('velocity of flow behind of moving shock= $\% 10.3 \mathrm{f} \mathrm{m} / \mathrm{s} \backslash \mathrm{n}$ ', Up);

fprintf('pressure behind shock wave (Region 2)=\%10.3f N/m2 \n',p2);

fprintf('pressure ahead shock wave (Region 1) $=\% 10.3 \mathrm{f} N / \mathrm{m} 2 \backslash \mathrm{n}$ ',p1);

fprintf('Tempreature behind moving shock wave (Region 2) $\% 10.3 f \mathrm{~K} \backslash \mathrm{n}$ ',T2);

fprintf('Tempreature ahead moving shock wave (Region 1) $\% 10.3 f \mathrm{~K} \backslash \mathrm{n}$ ', T1);

fprintf('Density of flow behind shock wave (Region 2) $=\% 10.3 \mathrm{f} \mathrm{kg} / \mathrm{m} 3$

$\backslash n^{\prime}$, Rho2);

fprintf('Density of flow ahead shock wave (Region 1) $=\% 10.3 \mathrm{f} \mathrm{kg} / \mathrm{m} 3$

$\backslash n^{\prime}$, Rho1);

DMin $=1.67 e-6 ; \operatorname{DMax}=11.7 e-6$;

!\% check liquid vapour phase change phenomena this equation is afunction of

$! \% \mathrm{~T}$ in celcious and surface tension in $\mathrm{mN} / \mathrm{m} 2$ so that we multiply the

result

!\% with $1.0 e-3$ and $T$ is subtracted from 273.15

sigma $=@(x)(75.83-0.1477 *(x-273.15)) * 1.0 e-3$;

fprintf('surface Tension= $\% 10.3 f \mathrm{~N} / \mathrm{m} 2 \backslash \mathrm{n}$ ', sigma(T1));

fprintf('minWeberNo= \%10.3f -/- \n', Rho1*Up^2*DMin/sigma(T1));

fprintf('maxWeberNo= \%10.3f $\left.-/-\backslash \mathrm{n}^{\prime}, \mathrm{Rho} 1 * \mathrm{Up} \wedge 2 * \operatorname{DMax} / \operatorname{sigma}(\mathrm{T} 1)\right)$;

!\% Lets find stagnation properties at this point

$\mathrm{T} 02=\mathrm{T} 2+\mathrm{Up}^{\wedge} 2 /(2 * \mathrm{Cp} 1)$;

$\mathrm{p} 02=\mathrm{p} 2+1 / 2 * \mathrm{Rh} O 2 * \mathrm{Up}^{\wedge} 2$;

Rho02=p02/(R1*T02);

fprintf('Total Temperature(stagnation Temp.)=',T02)

fprintf('Total pressure(stagnation Pressure)=',p02) 


\title{
A.2 Calculations of Atwood Number
}

\author{
PROGRAM randomCopy \\ !This Module has logGen Function and sizing array subroutines \\ USE sahirModule \\ ! $\quad \% \% \% \% \% \% \% \% \% \% \% \% \% \% \% \% \% \% \% \% \% \% \% \% \% \% \% \% \% \% \% \% \% \% \% \% \% \% \% \% \% \% \% \% \% \% \% \% \% \% \% \% \% \% \% \% \% \% \% \% \% \% \% \% \% \% \% \% \%$ \\ ! \% considering Actual situation in shock tube after running couple of \\ ! \% experiment the initial conditions might be slightly different for this \\ ! $\%$ reason we will say that we have $\% 50$ Air mixed with $\% 50$ N2 plus \\ ! \% relative humidity equals to $\% 15$ and so starting from Tdry=20 $\mathrm{C}$ and \\ ! \% pressure 101325 pa we can find our we will repeat most of previous \\ ! \% calculations.

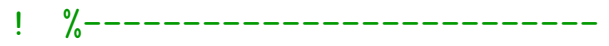 \\ ! Data Entry:\% \\ $! \%$ \\ !first case big size particles \\ REAL,DIMENSION(12)::diamInMicro ! 9 is the size of experemental data \\ defined below change to 12 if you use small particles \\ INTEGER,DIMENSION(12): : frequencyUltra $\quad 9$ is the size of experemental \\ data defined below change to 12 if you use small particles \\ REAL,DIMENSION (:), allocatable : :diamInMicro_tot, Theta \\ , R_data, XL, YL, randArea, $r$ \\ INTEGER : : diam_size, sample_size,ind,parcel \\ REAL:: MeanOfUltra, GeometricMean ,LogsigofUltra \\ REAL : : GeometricSTD, calcAtw, Pvsat, Rh,Pv,Pair, AreaOfInter \\ REAL : : MwMisd,airDens, PsatAir, Mw, RhoSat, volumeOf Inte \\ REAL: : volumeOfps,mOfParts, TotalmOHumidAir, RhoHav \\ REAL,DIMENSION (1): : smart1 !temprory storage has a dimension 1 or any \\ depends on logNormalDistribution
}




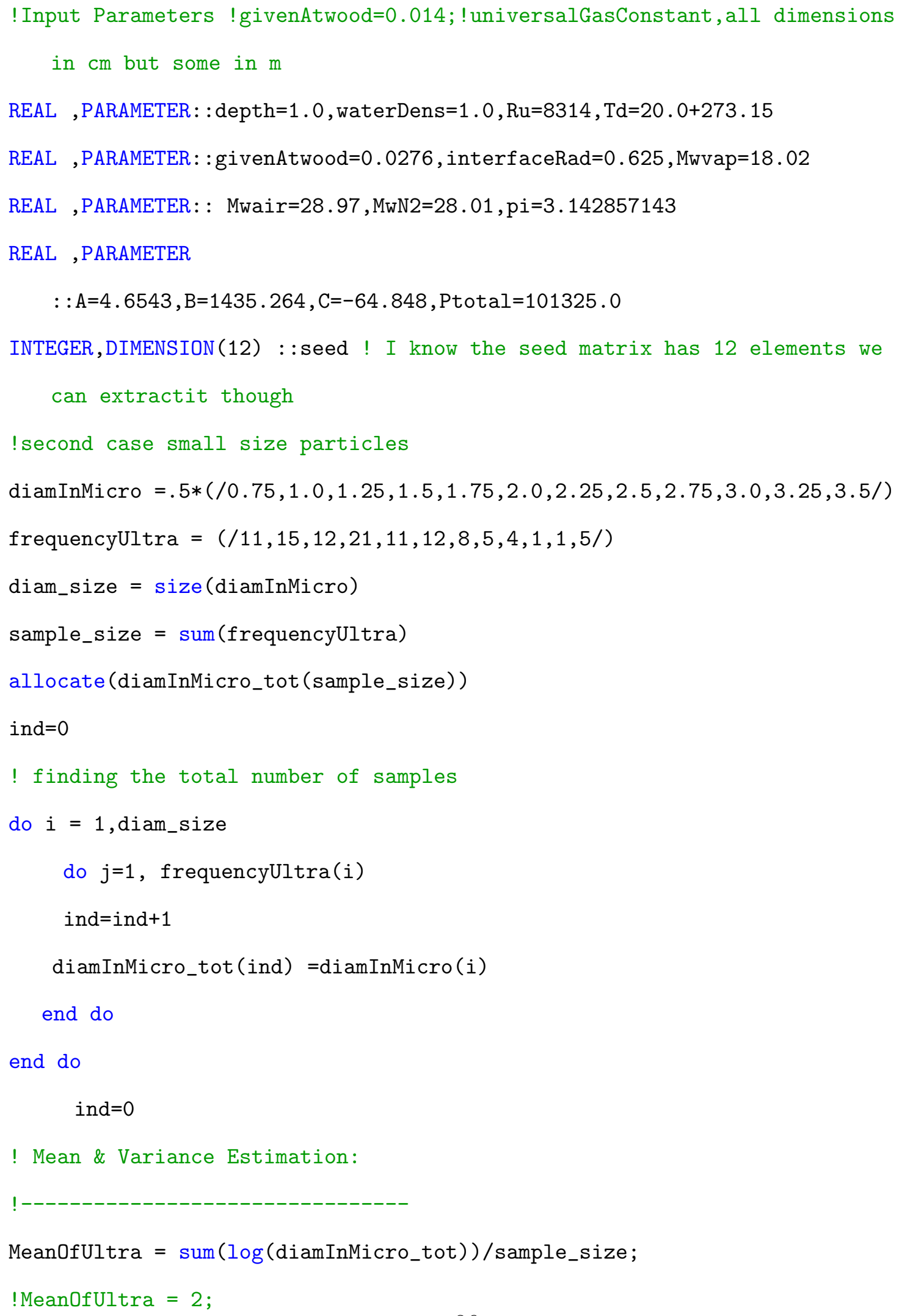




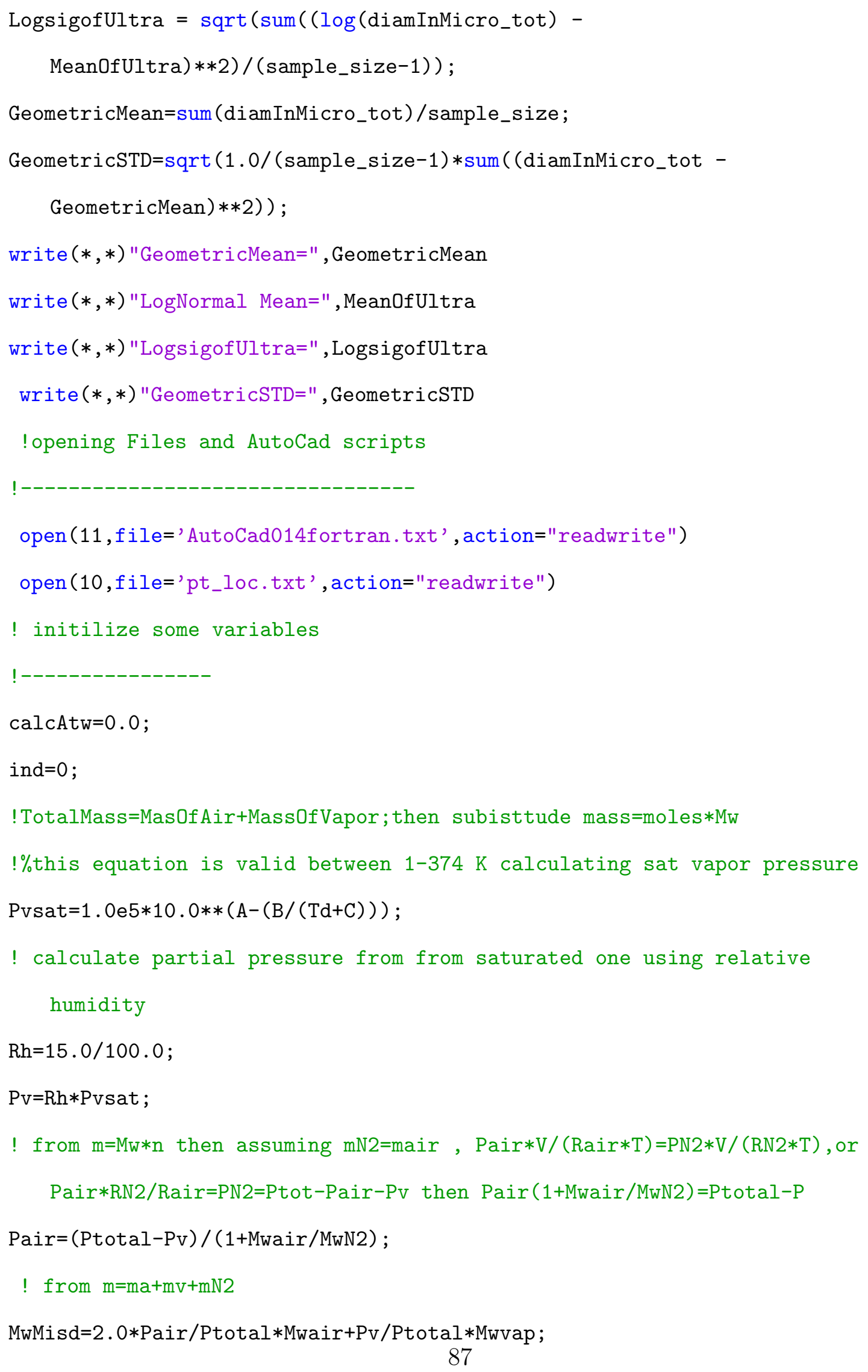




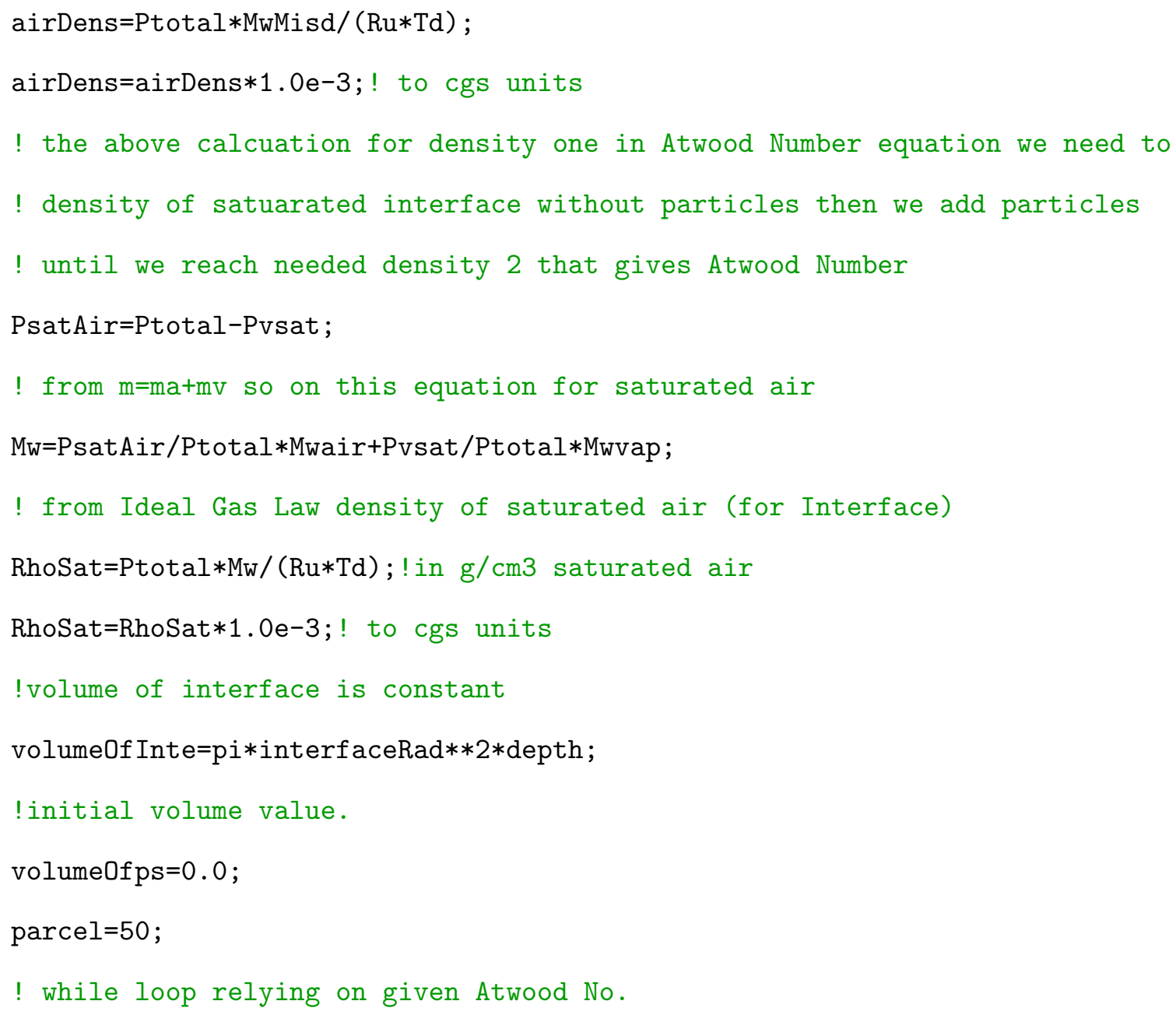


! notice here the attribute of smart1 should be an array withe same size of R_data (ind) $=\operatorname{smart1}(1)$

! returned value from the function above which was only one element volumeOfps=volumeOfps + parcel $* 4.0 / 3.0 * p i * R \_$data $($ind $) * * 3$;

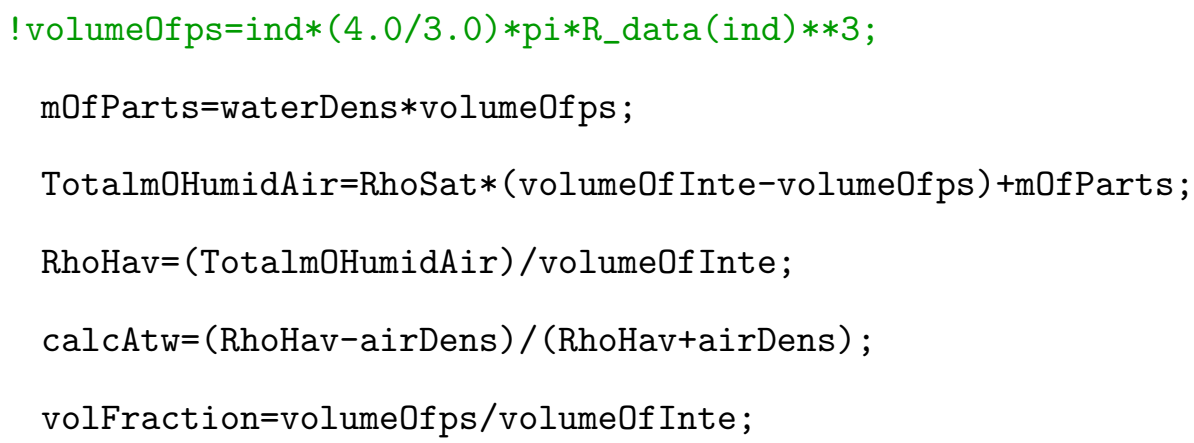

end do

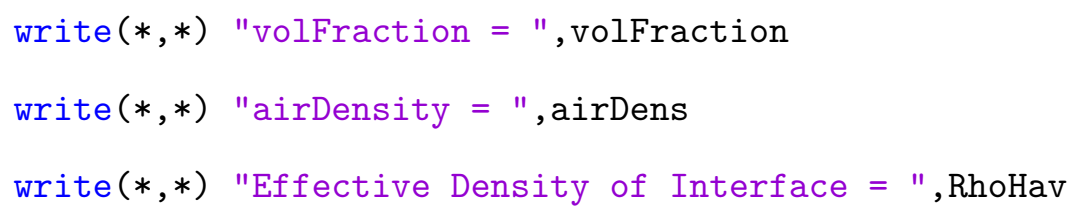




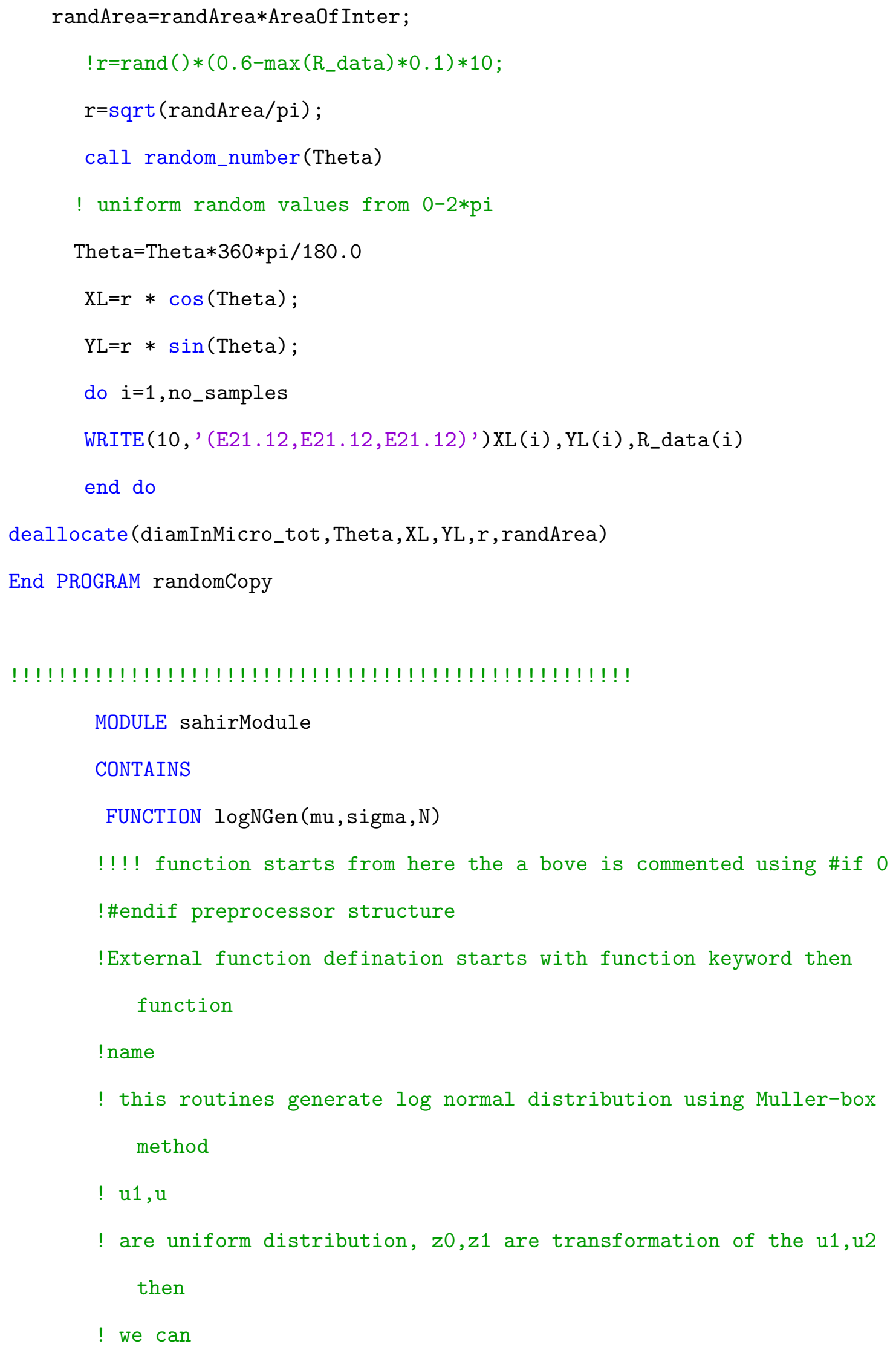




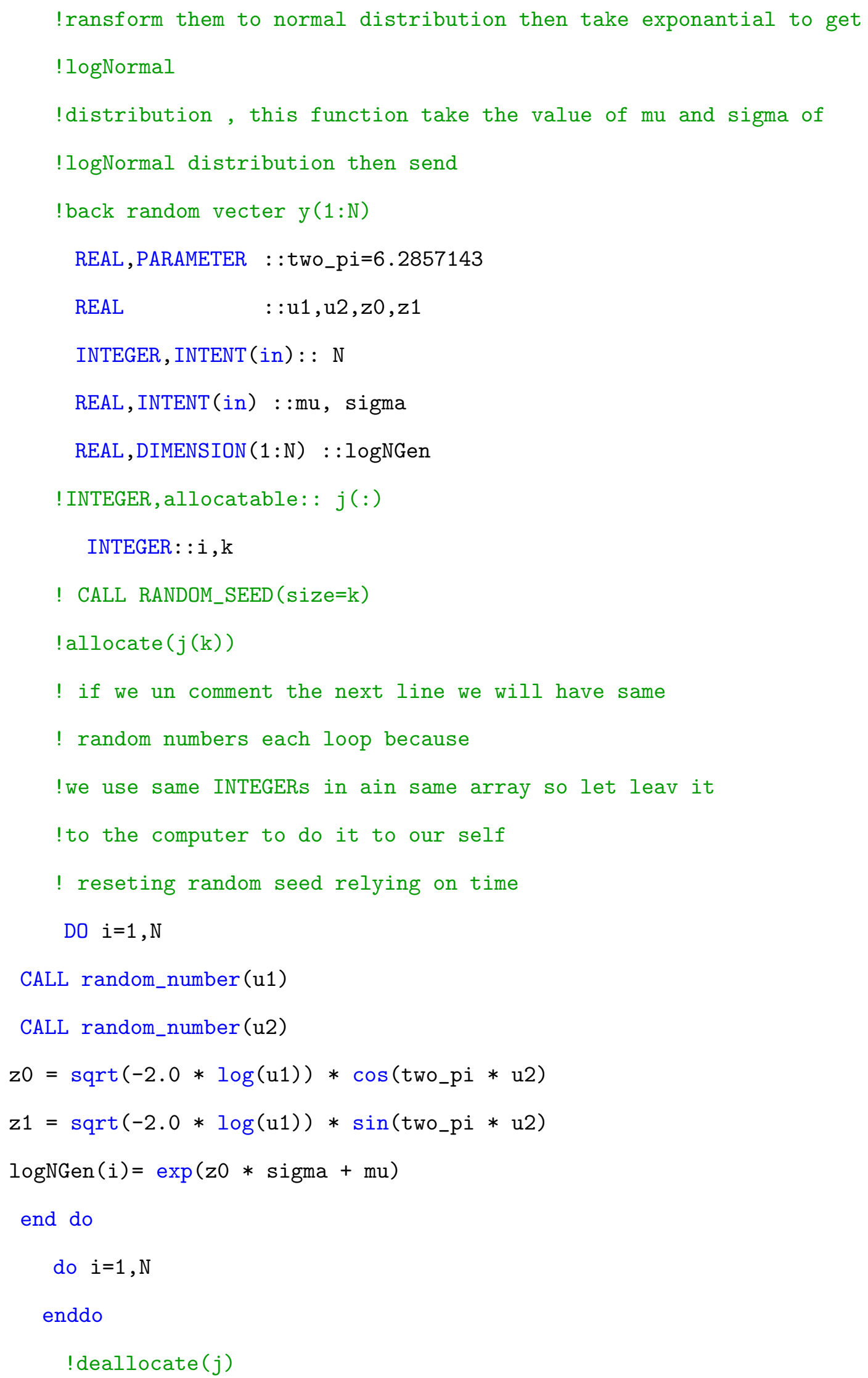


end function logNGen

! ! !CALL RANDOM_SEED $(\operatorname{size}=j)$ ! ! !

! this subroutines has 3 cases

!

! CALL RANDOM_SEED $(\operatorname{size}=j)$ gives us the size of default vector

! Array or vector matrix used to generate

! random number as you know generation random numbers rely on a

! seed (starting point of generation) and we can use

! CALL RANDOM_SEED (put $=(/ 1,2,3,4,5,6,7,8,9,10,11,12 /))$ to put our

! matrix that we can use to generate same random numbers

! CALL RANDOM_SEED(get=seed) gives us the the values of default

! matrix that we can change using put as above

!

SUBROUTINE resize_array(array, ind)

! this routine resizing the array from big to small size by trimming the

! zeros

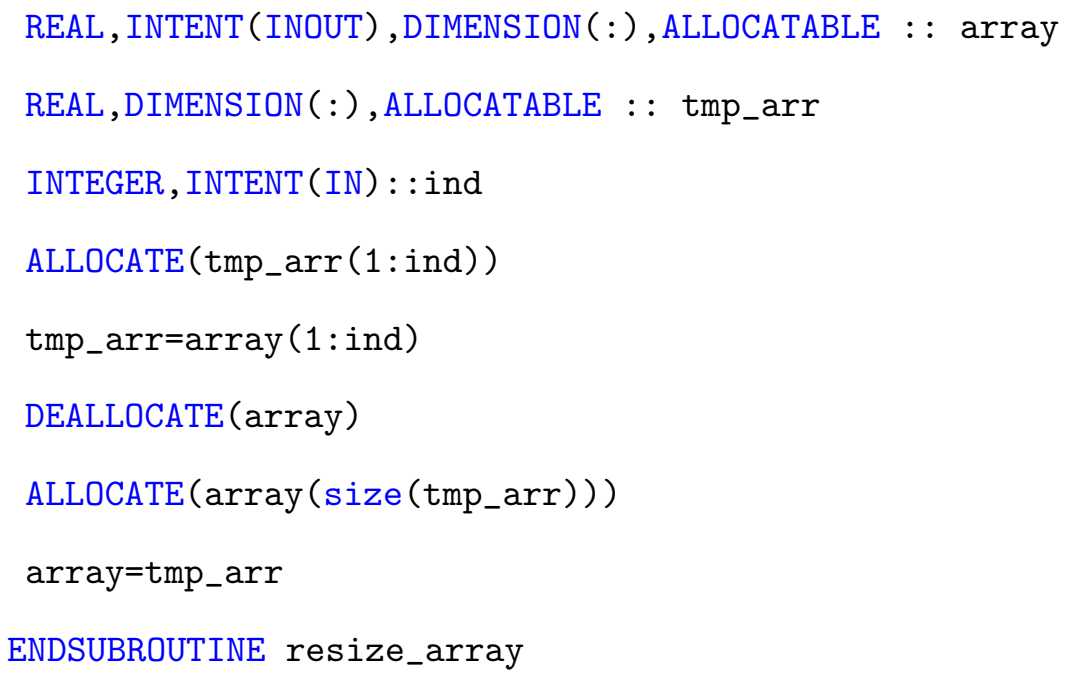

End MODULE sahirModule 


\section{A.3 Non-Continuum Drag Model}

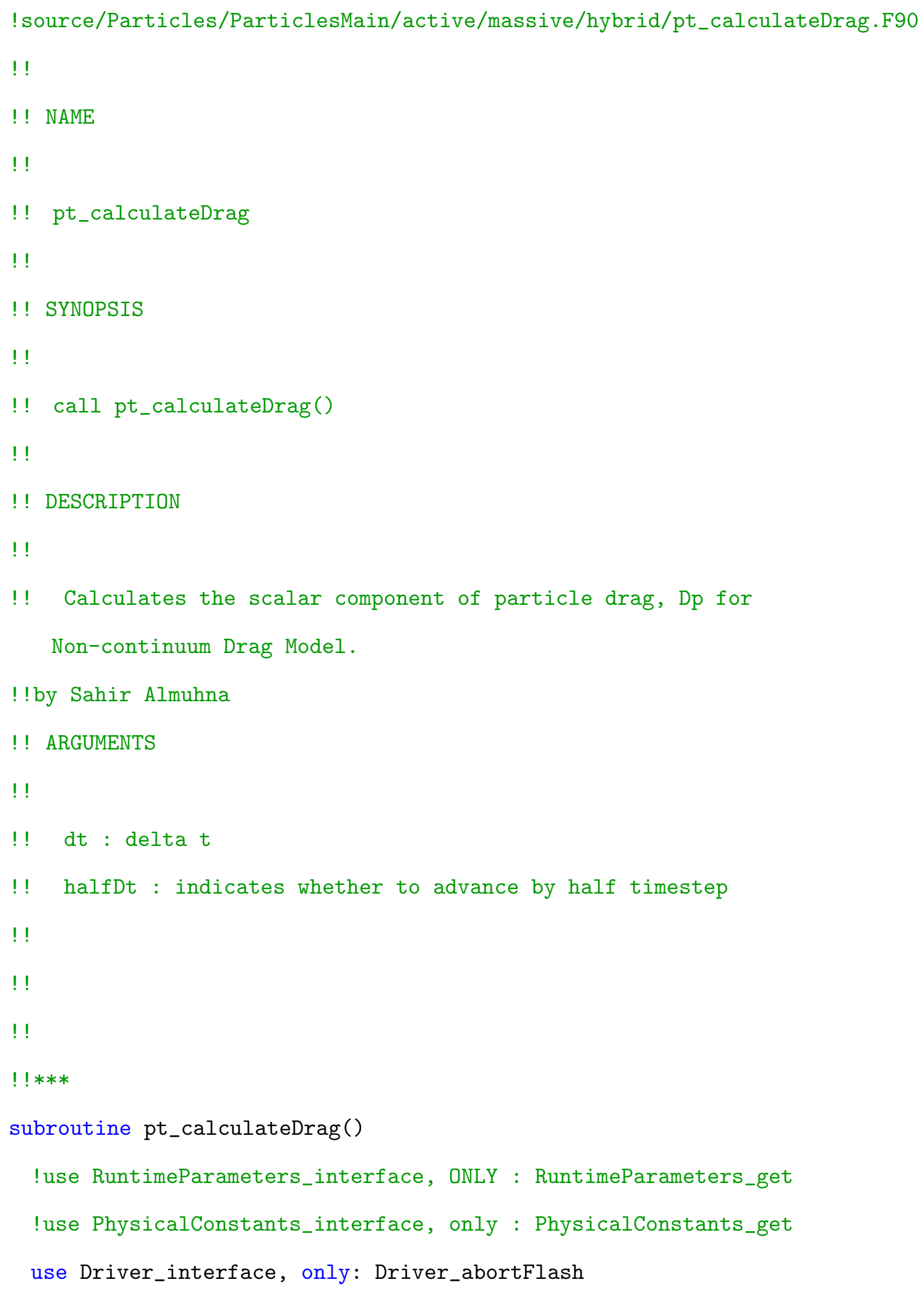




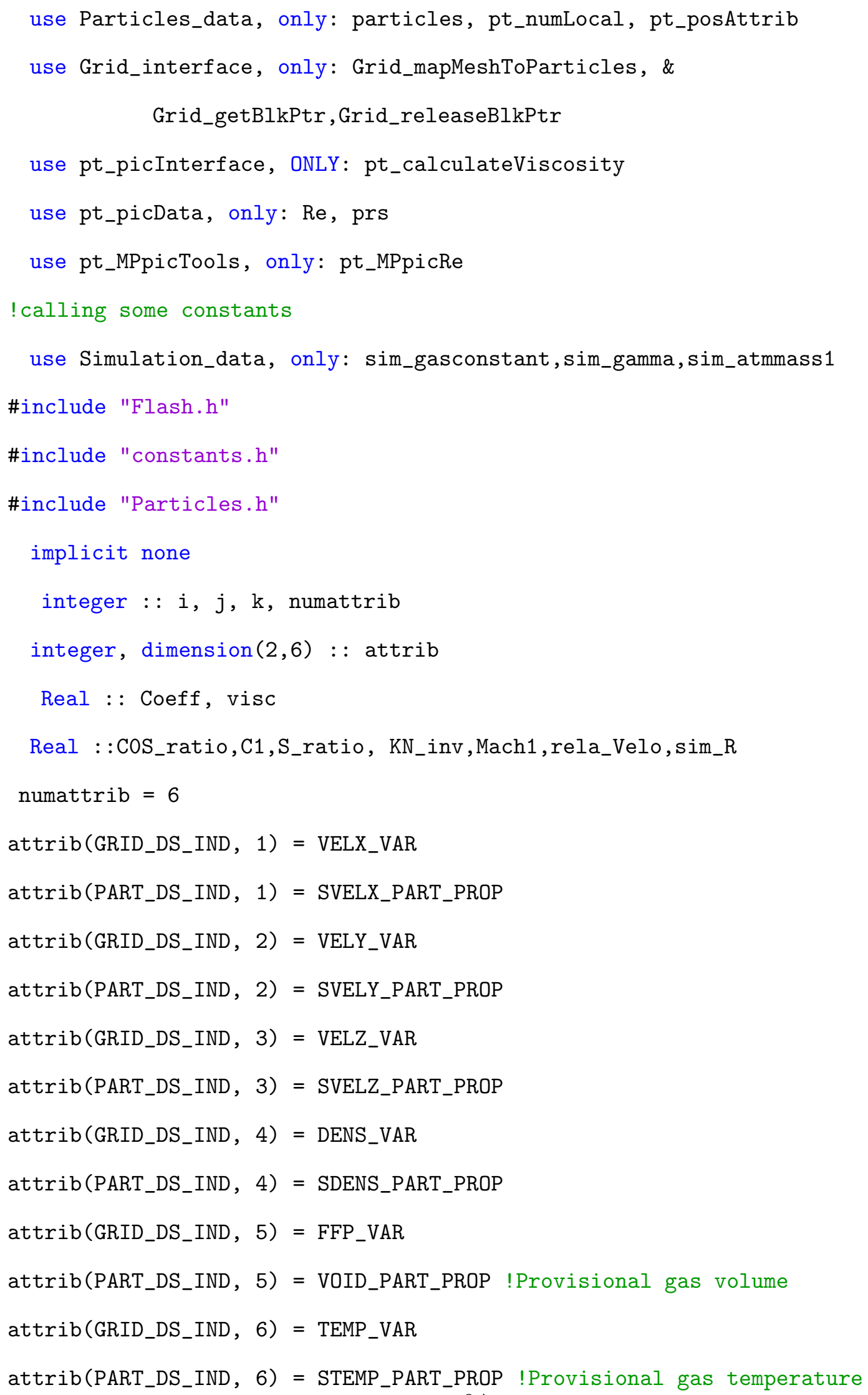




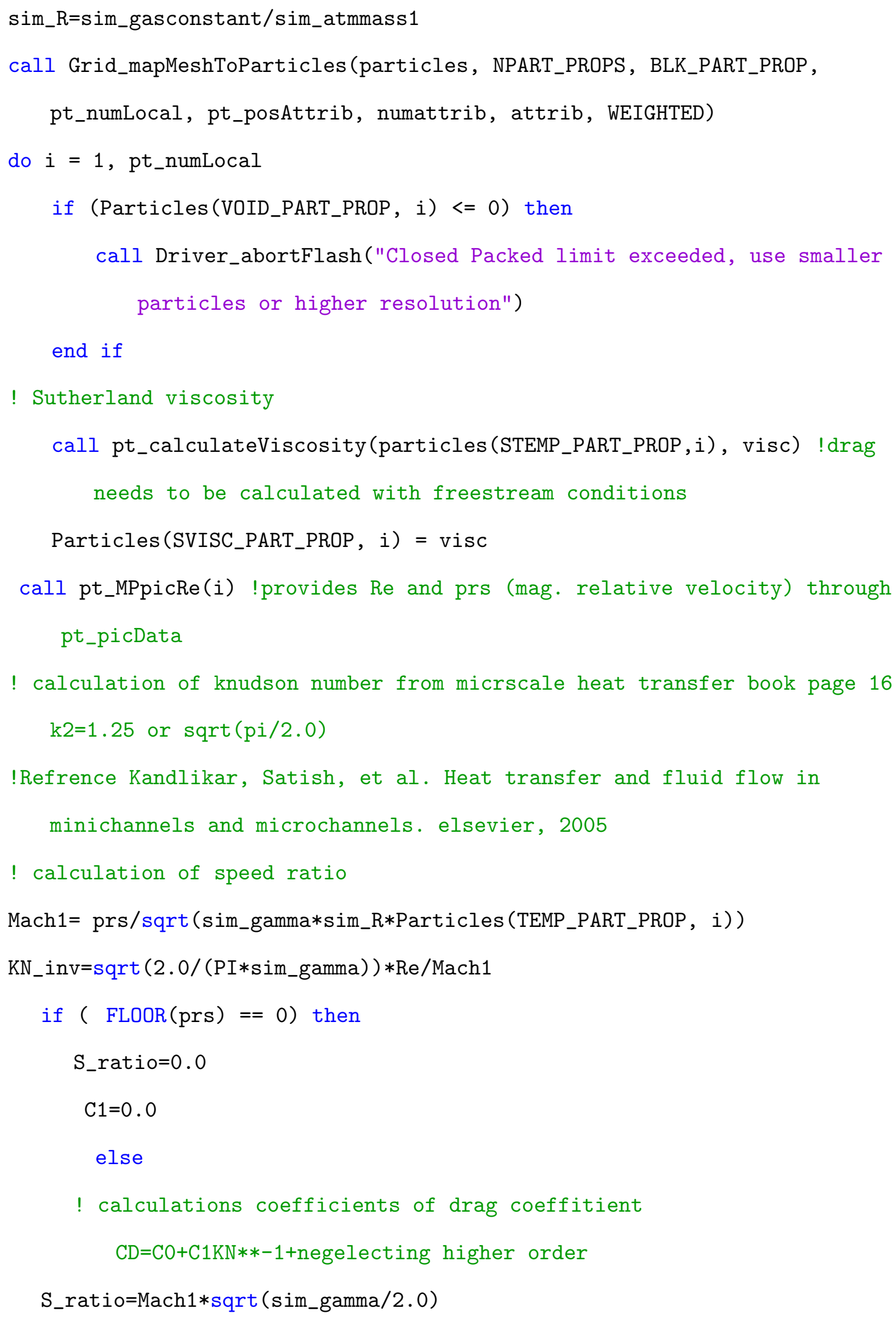




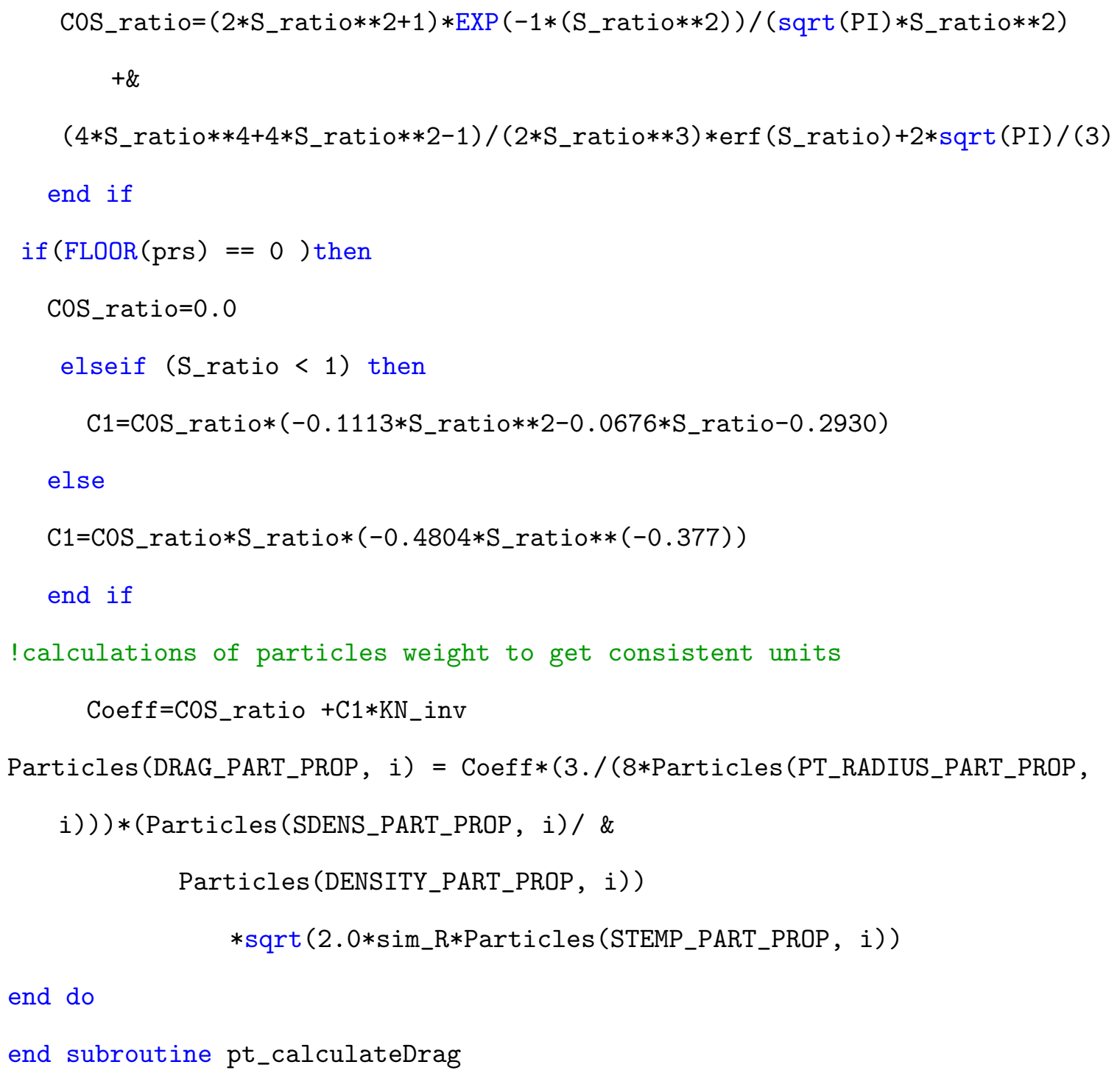

\section{A.4 Point mass Gravity Model}

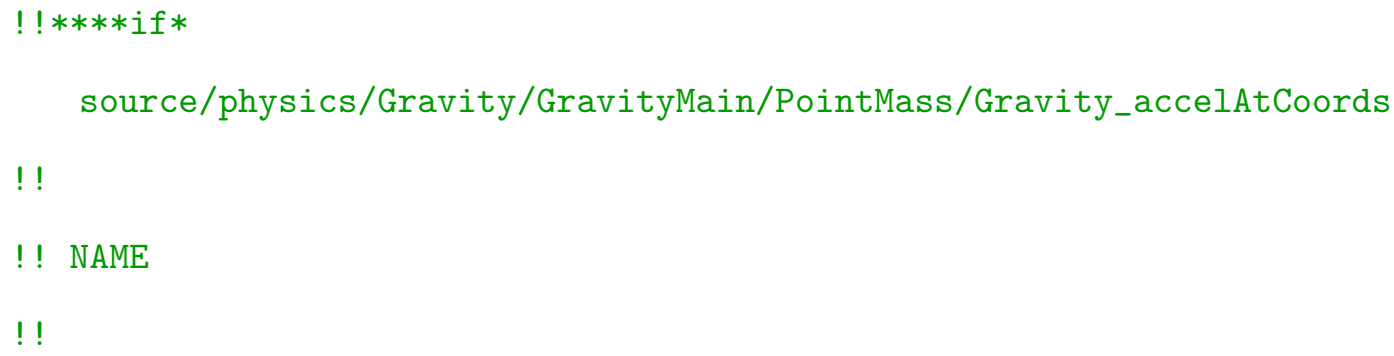




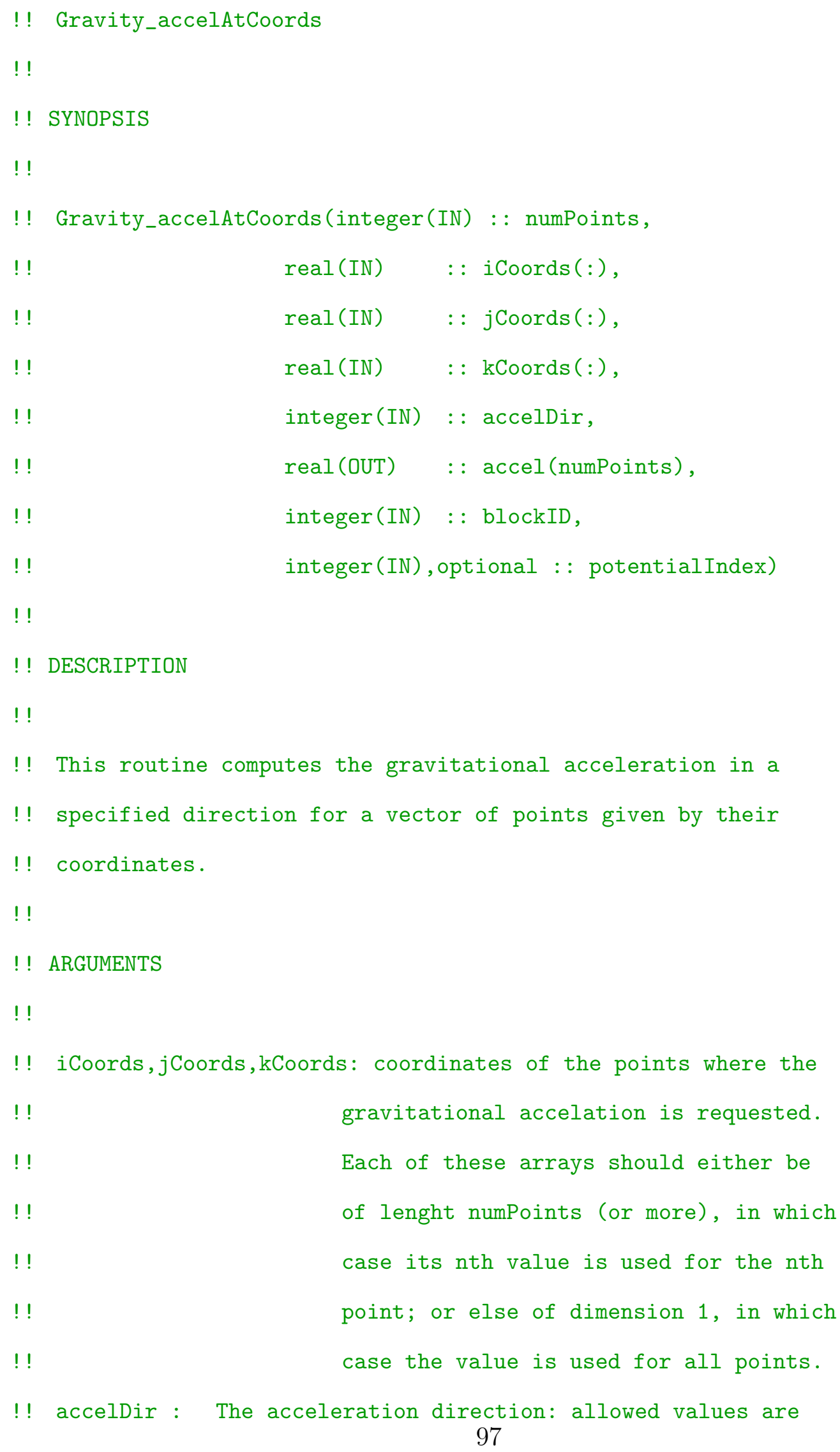
Each of these arrays should either be of lenght numPoints (or more), in which case its nth value is used for the nth point; or else of dimension 1, in which 


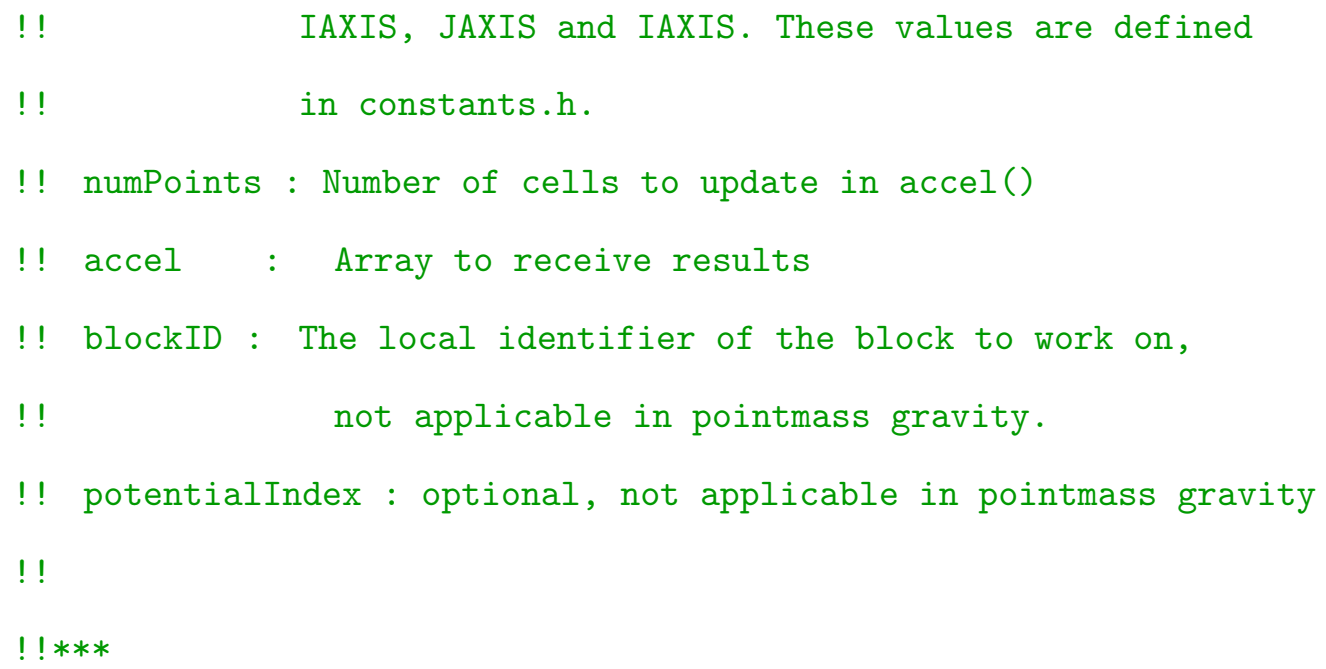




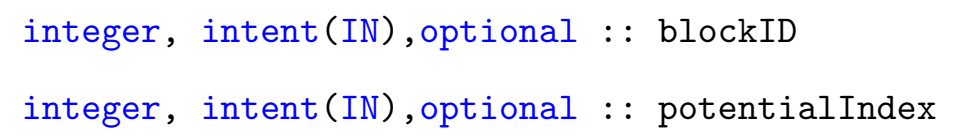




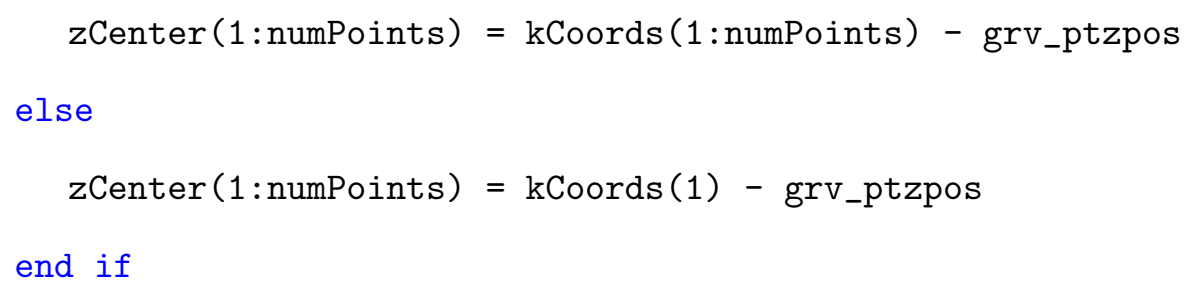




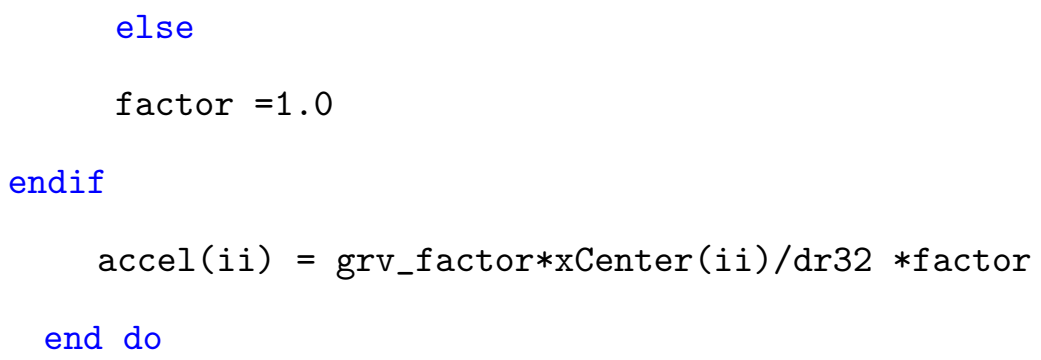




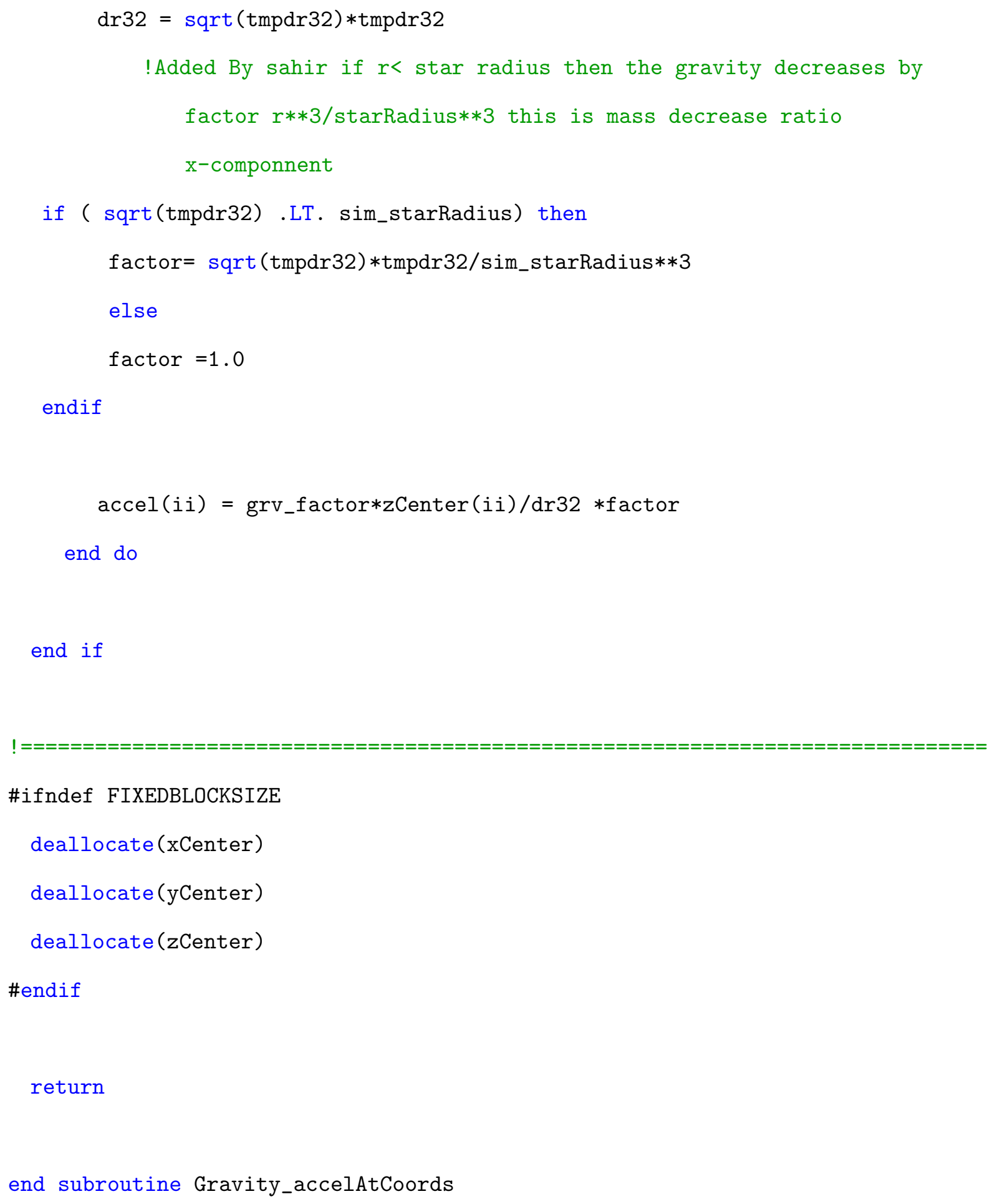




\section{A.5 Input Parameters}

!\# flash.par file All parameters included in Config files for simulations can be changed here

useParticlesGravity=.true.

useGravity $\quad=$.true.

shockDetect $=$. false.

use_steepening $=$.false.

use_cma_flattening $=$.true .

ppm_modifystates $=$.true

gamma $=1.41$

pc_unitsBase

$=$ "CGS"

!\# End of simulation, max no. of iteration and maximum time. (Simulation ends whichever ! is reached first)

nend $=300000$

$\operatorname{tmax}=200 e 8$

!\# checkpoint file output parameters, how frequently should the results be dumped into !checkpoint files

checkpointFileIntervalTime $=1.0 e 6$

particleFileIntervalTime $=1.0 e 6$

checkpointFileNumber $=0$

particleFileNumber $=0$

!\# plotfile output parameters

plotfileIntervalTime $=0$.

plotfileIntervalStep $=0$

plotfileNumber $=0$

center $x=3.75 e 14$

centery $=3.75 e 14$

Irefine_min $=1$ 


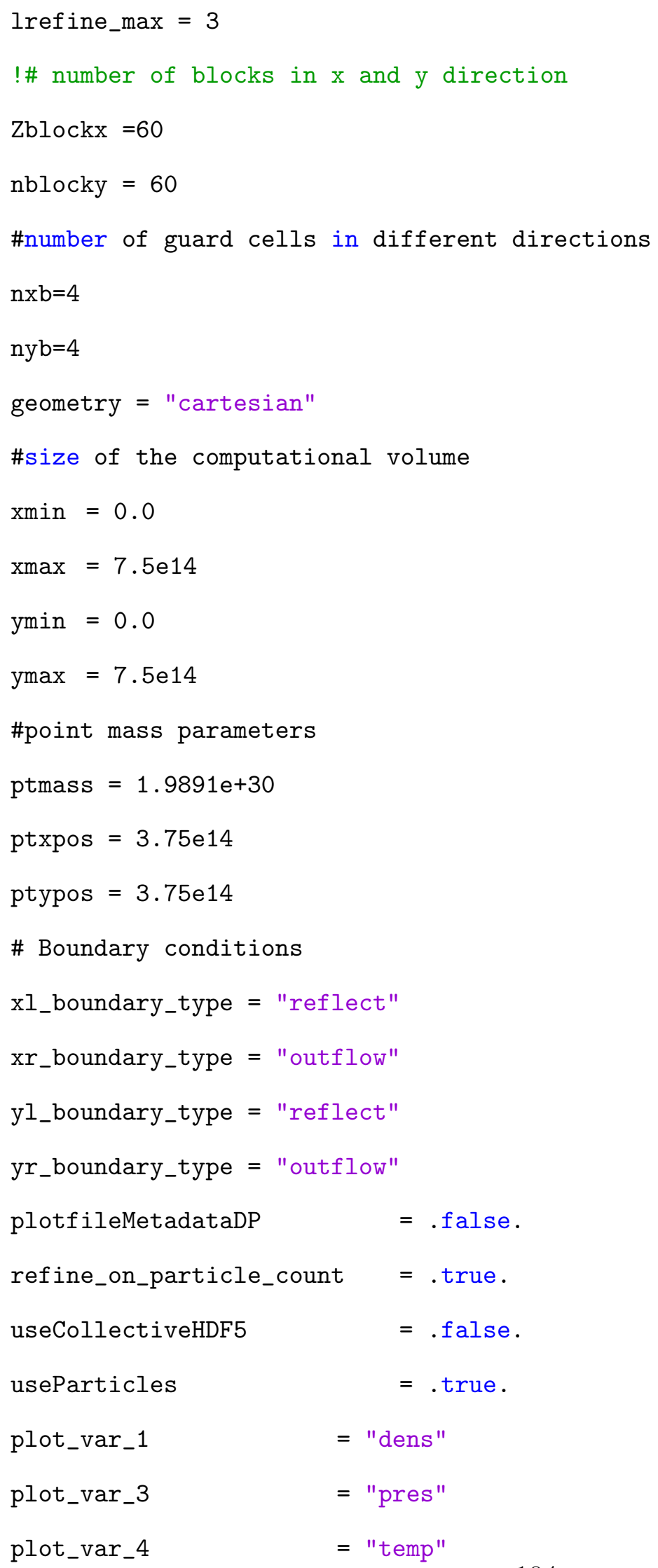




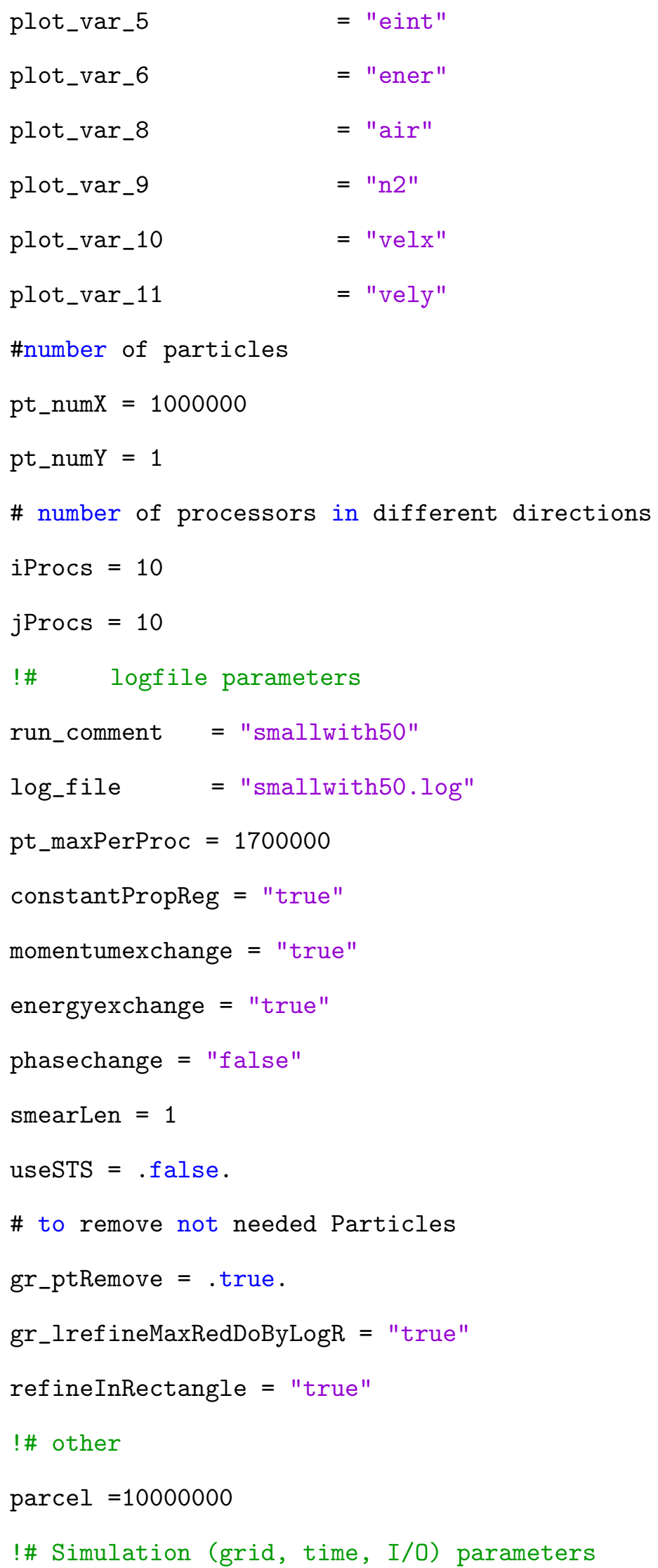




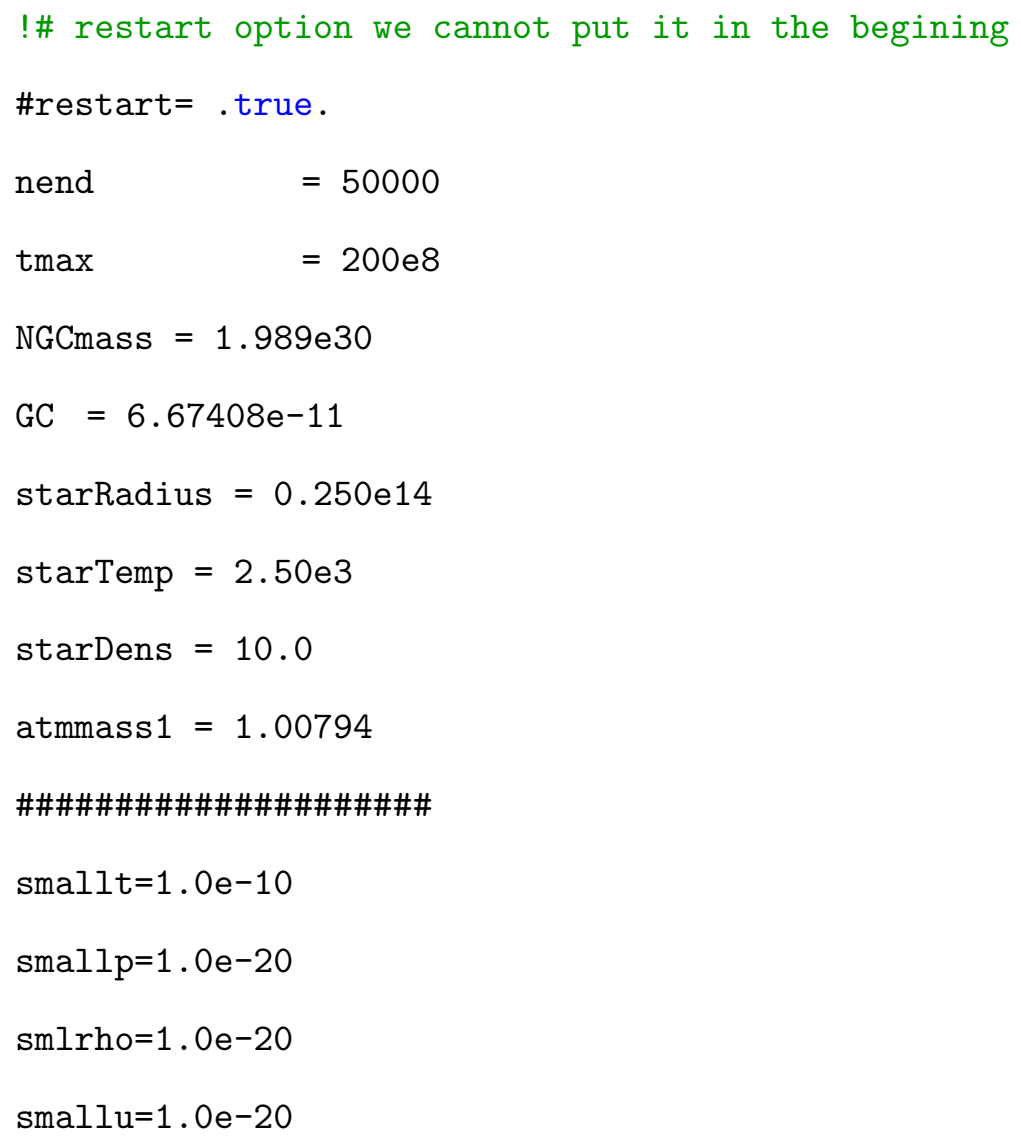




\section{VITA}

I have started my education in the University of Babylon/Iraq in 1996, and I got my bachelor degree in Mechanical engineering. Then, I got a master science in the same department (2000-2004). I have taught different mechanical engineering classes as a university professor; for example, Static, Dynamic, Fluid flow lab, MathII, and Numerical Solution Methods. I got married after getting my master degree in 2005. I Joined University of Missouri as a PhD candidate in 2015 and from that time till now I spent most of my time to finish the $\mathrm{PhD}$ requirements until I got my $\mathrm{PhD}$ degree. I enjoy my leisure time with my family. My plan is to stay in academia teaching in a Mechanical Engineering school. 